Florida International University FIU Digital Commons

\title{
The Development and Initial Validation of a Self- Assessment for Global Leadership Competencies
}

Sabrena A. O'Keefe

Florida International University, saokeefe@fiu.edu

DOI: $10.25148 /$ etd.FIDC006566

Follow this and additional works at: https://digitalcommons.fiu.edu/etd

Part of the Educational Assessment, Evaluation, and Research Commons, Educational

Leadership Commons, Leadership Studies Commons, and the Other International and Area Studies

Commons

\section{Recommended Citation}

O'Keefe, Sabrena A., "The Development and Initial Validation of a Self-Assessment for Global Leadership Competencies" (2018). FIU Electronic Theses and Dissertations. 3694.

https://digitalcommons.fiu.edu/etd/3694 


\section{FLORIDA INTERNATIONAL UNIVERSITY}

Miami, Florida

\section{THE DEVELOPMENT AND INITIAL VALIDATION OF A SELF-ASSESSMENT FOR GLOBAL LEADERSHIP COMPETENCIES}

A dissertation submitted in partial fulfillment of

the requirements for the degree of

DOCTOR OF PHILOSOPHY

in

HIGHER EDUCATION

by

Sabrena O'Keefe 
To: Dean Michael R. Heithaus

College of Arts, Sciences and Education

This dissertation, written by Sabrena O'Keefe, and entitled The Development and Initial Validation of a Self-Assessment for Global Leadership Competencies, having been approved in respect to style and intellectual content, is referred to you for judgment.

We have read this dissertation and recommend that it be approved.

Haiying Long

Thomas G. Reio

Maria Lovett

Hilary Landorf, Major Professor

Date of Defense: March 21, 2018

The dissertation of Sabrena O'Keefe is approved.

Dean Michael R. Heithaus

College of Arts, Sciences and Education

Andrés G. Gil

Vice President for Research and Economic Development and Dean of the University Graduate School

Florida International University, 2018 
(C) Copyright 2018 by Sabrena O'Keefe

All rights reserved. 


\section{DEDICATION}

To my mother, Pamela O'Keefe, because her early death inspires me to live like I'll die tomorrow, but learn like I'll live forever. 


\section{ACKNOWLEDGMENTS}

First and foremost, I must thank my committee whom guided and supported me through this process - Dr. Hilary Landorf, Dr. Haiying Long, Dr. Thomas Reio, and Dr. Maria Lovett. I offer extra appreciation for my chair Dr. Landorf with whom I spent many hours discussing global leadership concepts while trying to get my theoretical concepts complete as I was writing Chapters 1 and 2, and my methodologist Dr. Long who was essential in pushing/helping me make Chapters 3 and 4 richer and deeper - even if that meant late night calls and quick feedback turn arounds for me to be able to meet my defense deadlines.

I would also like to thank all the faculty that helped me along the way. While I started this journey with just the end in mind, I was truly transformed along the way because of them. I'll never forget my first doctoral class with the late Dr. Bob Farrell, who I thought was a little crazy, but credit for helping me begin to think critically at a higher level. After that Dr. Linda Bliss, Dr. Joy Blanchard, Dr. Dawn Addy, Dr. Diann Newman, Dr. Benjamin Baez, Dr. Mido Chang, Dr. Cathy Akens, and Dr. Haiying Long all continued to challenge me in very different ways, contributing to my growth as an academic.

I'll be forever grateful for Dr. Corey Seemiller. Her Student Leadership Competencies research is the backbone to the many projects that have allowed me to enhance experiences for students, and especially to my dissertation. Her counsel in the early stages of my instrument development really helped put me on the right track and the feedback she provided when she served as an expert during phase one of my study was invaluable. 
I also want to thank the others that served as experts during phase one: Dr. Beverly Dalrymple, Dr. Hilary Landorf, Dr. Thomas Reio, Dr. Maria Lovett, Dr. Stephanie Doscher, Eric Feldman, Patricia Lopez-Guerrero, Joanna Garcia, Nashira Williams, Kaleen Martinez, Peter Melnik, and Shannonlee Rodriguez. I know it took them all a significant amount of time to fill out both tables of specifications, and their input was essential to my process. Another person that dedicated much time to help me in my process was Emani Jerome, who participated as a second researcher during phase two of my process. I appreciated his thoughtful inquires and analysis throughout that process.

I have been blessed to have many different support systems as I have moved through this process. Starting with my GRE study group Michelle Castro, Nikki Kogan, and Franklin McCune. Then my mini-cohort: Dr. Bronwen Bares Pelaez, Dr. Carolyn Meeker, Yselande Pierre, Nashira Williams, and many more. I appreciate that misery loves company and we have all been able to be there for each other through the ups and downs of this process. FIU in general has been a very supportive place to work while completing my doctoral degree and I'll be forever grateful to the institution and in particular my office for that support. I must also give a shout out my incredible network of colleagues across the country that helped recruit students to take my instrument during my final phase, I literally could not have completed this process without them!

Additionally, I would be nowhere without the unconditional love and support of my friends and family. Thank you for always being there to listen and for your patience with me. Even when you had no idea what exactly I was going through, you did your best to either be there or leave me alone as necessary. There were many sacrifices each 
of you had to make in order for me to be successful and I truly appreciate your willingness to make them.

Most importantly I need to thank my sister and best friend, Carren O'Keefe, father, Raymond O'Keefe, and stepmother, Crystal O'Keefe. Even from far away the three of you have been my biggest cheerleaders. When I did not believe I could do this, the three of you never doubted my capabilities. Everything I am is because of you three (and mom who has been watching over us). I can never thank you enough.

All of you, and many more, have empowered me to achieve this accomplishment. I am so lucky to have each and every one of you in my life. Thank you again from the bottom of my heart. 
ABSTRACT OF THE DISSERTATION

THE DEVELOPMENT AND INITIAL VALIDATION OF A SELF-ASSESSMENT

FOR GLOBAL LEADERSHIP COMPETENCIES

\author{
by \\ Sabrena O'Keefe \\ Florida International University, 2018 \\ Miami, Florida \\ Professor Hilary Landorf, Major Professor
}

Global leadership has been a growing area of research as our world becomes interconnected. The National Association for College Employers (NACE) Career Readiness Competencies Work Group even added an eighth competency: global/intercultural fluency. Employers have also expressed a skill crisis regarding students graduating from college without the necessary global leadership skills. However, there are often not enough resources at institutions of higher education to add specific co-curricular programs around global leadership. At the same time many institutions have begun to use the Student Leadership Competencies (Seemiller, 2013) as learning outcomes for their co-curricular programs.

This research study aimed to combine the concepts of global leadership and the Student Leadership Competencies so that students have the opportunity to develop global leadership competencies on their own. The researcher created a global leadership competencies self-assessment instrument mapped within the Student Leadership Competencies, then demonstrated the extent to which the instrument yields evidence that supports valid and reliable inferences about students' global leadership competencies. 
Validity evidence based on content was established through the use of 13 subject matter experts. Validity evidence based on cognitive process was established through cognitive interviews. Validity evidence based on internal structure was established by conducting an exploratory factor analysis. Specifically, a principal axis factor analysis with a varimax rotation was conducted on data gathered from 279 participants. Evidence supported the finding that the instrument yielded reliable inferences about students' global leadership competencies ( 30 items; $\alpha=.932$ ).

There were six constructs uncovered and measured through the validation process: Interpersonal Impact, Perspective-taking, Adapting, Diversity, Responding to Ambiguity, and Resiliency. The instrument created in this study provides self-awareness of a student's proficiency in these global leadership competencies, which enables them to seek out development opportunities for those competencies either on their campus or in other out-of-the-classroom activities based on their results. This instrument can now be used to guide a students' global leadership competency development journey. 


\section{TABLE OF CONTENTS}

CHAPTER

PAGE

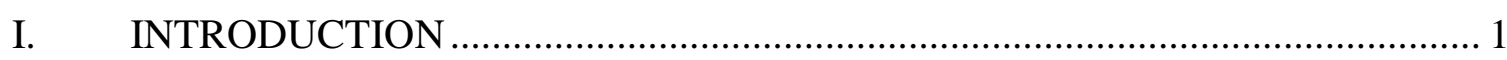

Background of the Study ………………………………............................. 1

Research Problem ............................................................................................ 11

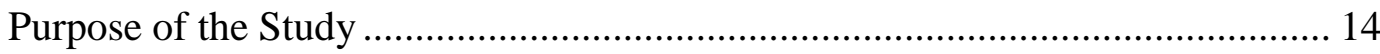

Research Questions ...................................................................................... 14

Theoretical Framework ……………................................................................. 14

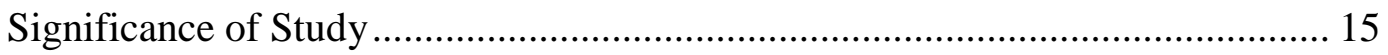

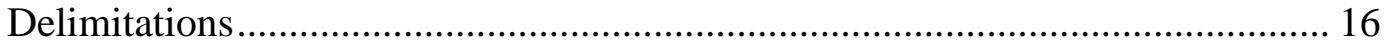

Assumptions............................................................................................ 16

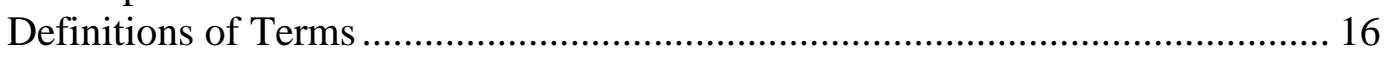

Overview of Succeeding Chapters ................................................................... 18

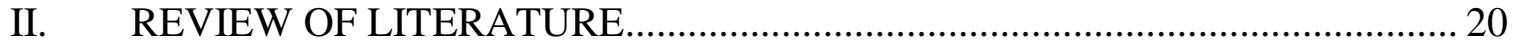

The Evolution of Global Leadership Literature................................................... 20

Global Leadership Competency Frameworks and Models ................................... 23

Global Leadership Assessments .................................................................... 30

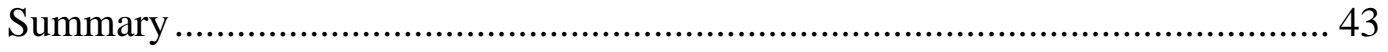

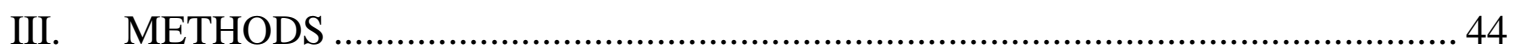

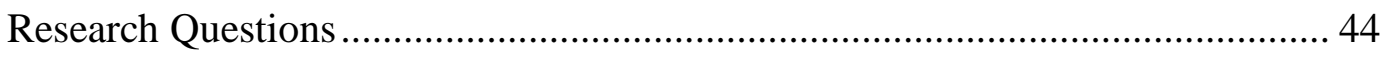

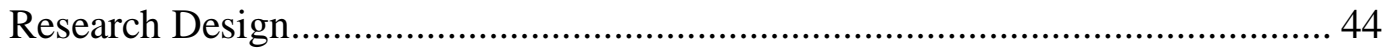

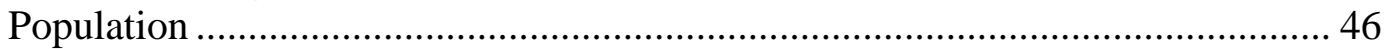

Relevant Conceptions of Validity and Reliability ................................................ 46

Data Collection Procedures and Methods ............................................................ 48

Data Analysis Procedures …………………………....................................... 59

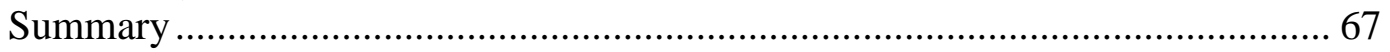

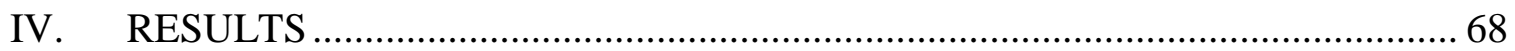

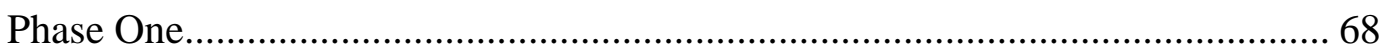

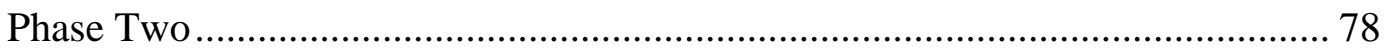

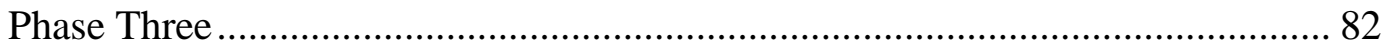

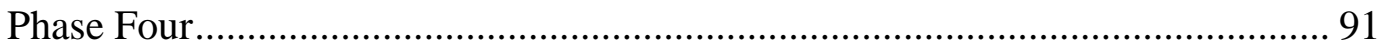

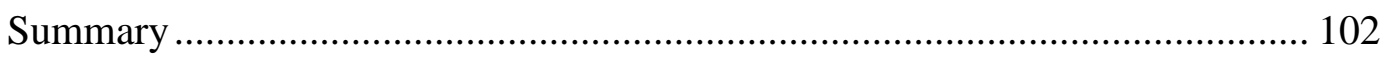

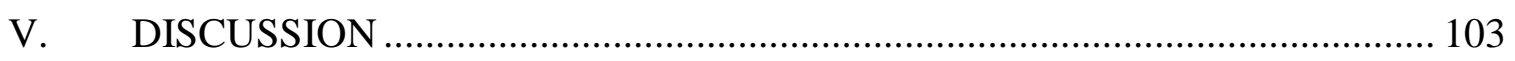

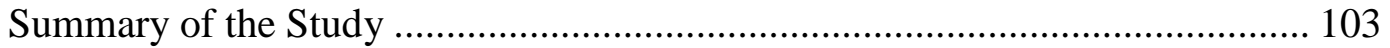

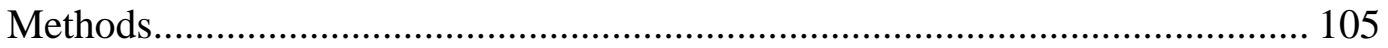

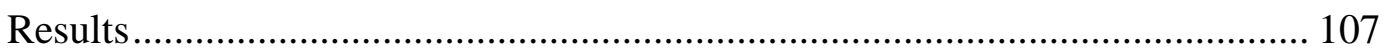

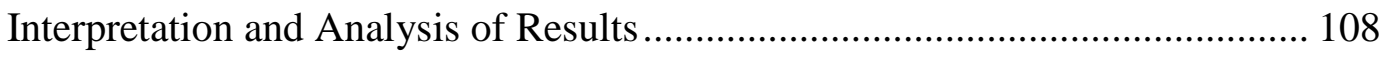

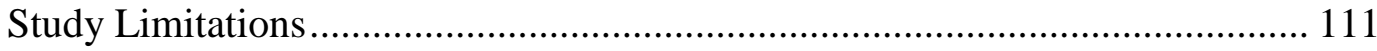

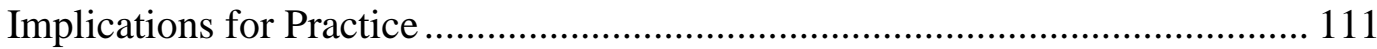


Recommendations for Future Research ................................................. 115

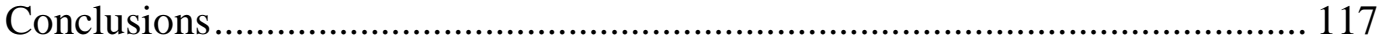

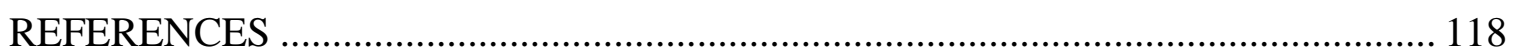

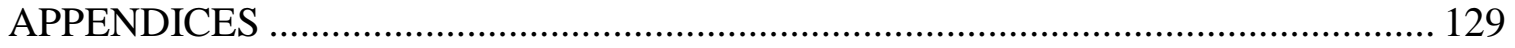

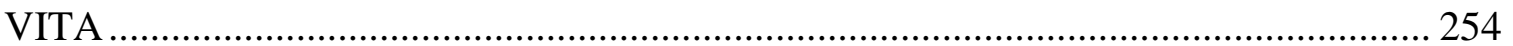




\section{LIST OF TABLES}

TABLE

PAGE

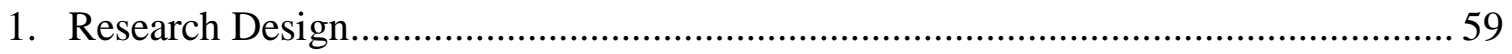

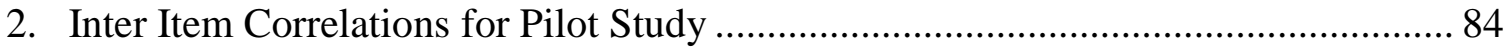

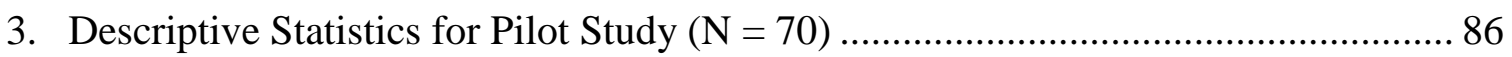

4. Summary of Exploratory Factor Analysis Results for Pilot Study $(\mathrm{N}=70)$............ 89

5. Descriptive Statistics for O'Keefe Global Leadership Assessment $(\mathrm{N}=279)$............. 92

6. Summary of Exploratory Factor Analysis Results for O’Keefe Global Leadership Assessment $(\mathrm{N}=279)$. 


\section{LIST OF FIGURES}

TABLE

PAGE

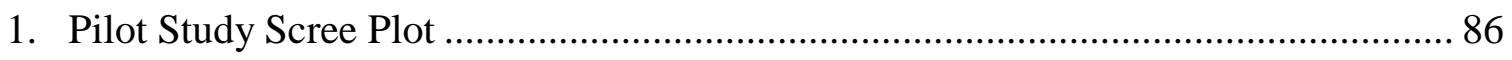

2. O'Keefe Global Leadership Assessment Scree Plot..................................................... 94

3. O'Keefe Global Leadership Assessment Collapsed Factors Scree Plot ..................... 95 


\section{LIST OF ACRONYMS}

ACE American Council on Education

AERA American Educational Research Association

APA American Psychological Association

BASIC Behavioral Assessment Scale for Intercultural Communication Effectiveness

BBC Biscayne Bay Campus of Florida International University

CAS Council for the Advancement of Standards

CCAI Cross-Cultural Adaptability Inventory

CCSS Cross-Cultural Sensitivity Scale

CLS Center for Leadership and Service

CQ Cultural Intelligence Scale

DiSC Personal Profile System

FIU Florida International University

GCI Global Competencies Inventory

GMI Global Mindset Inventory

IBA Intercultural Behavioral Assessment

ICAPS Intercultural Adjustment Potential Scale

ICC Intercultural Communication Competence

ICSI Cultural Intelligence Scale

IDI Intercultural Development Inventory

IS Intercultural Sensitivity Scale

LPI Leadership Practices Inventory

LSI Kolb Learning Style Inventory 


$\begin{array}{ll}\text { MBTI } & \text { Meyers-Briggs Typology Indicator } \\ \text { MMC } & \text { Modesto Maidique Campus of Florida International University } \\ \text { MPQ } & \text { Multicultural Personality Inventory } \\ \text { NACE } & \text { National Association for College Employers } \\ \text { NAFSA } & \text { Association for International Educators } \\ \text { NCME } & \text { National Council on Measurement in Education } \\ \text { SLC } & \text { Student Leadership Competencies } \\ \text { SRLS } & \text { Socially Responsible Leadership Scale } \\ \text { TC } & \text { True Colors }\end{array}$




\section{CHAPTER I}

\section{INTRODUCTION}

The purpose of this study is to develop a self-assessment instrument with psychometrically sound reliability and validity inferences to measure global leadership competencies. Specifically, it presents a two-stage approach: (a) the identification of scale items that reflect global leadership competencies, which addresses evidence of test content and cognitive response aspects of validity, and (b) the discovery of evidence for internal structure, as well as, reliability estimates of the developed scale items. Chapter 1 provides the background of the proposed study, the research problem and purpose, research questions, the theoretical framework, and the study's significance, assumptions, and delimitations. The chapter concludes with definitions of terms and an overview of succeeding chapters.

\section{Background of the Study}

For decades, institutions of higher education across the United States have offered co-curricular activities that help undergraduate students develop leadership capabilities (Astin \& Astin, 2000; Hamrick, Evans, \& Schuh, 2002; National Association of Student Personnel Administrators, 2016). Leadership seminars and workshops, mentoring programs, guest speakers, service projects, outdoor education, and leadership courses have been used to teach communication, problem-solving, personal and social responsibility, vision, conflict resolution, and other leadership related topics (Zimmerman-Oster \& Burkhardt, 2000). More recently this area has expanded to include leadership that incorporates global or intercultural competencies. In 2002, the American Council on Education (ACE) released a report that pointed out the shortcomings of the 
nation's international expertise and citizens' understanding of other cultures and global affairs. In 2007, the Association of American Colleges and Universities (AAC\&U) after conducting an employer survey, determined that there was a need for global competence development for students. A study by Ghasabeh, Soosay, and Reaiche on transformational leadership stated, “today's globalized nature of competitiveness is placing more pressure on organizations to employ effective leaders who are capable to develop a global vision for organizations" (2015, p. 460). The need for global vision was again confirmed in 2017 when the National Association for College Employers (NACE) Career Readiness Competencies Work Group expanded the career-readiness competencies research originally conducted in 2014 with the addition of an eighth competency - global/intercultural fluency (NACE, 2017). Examples of competencies that require a higher level of proficiency for people desiring to be global leaders include ability to adapt, willingness to learn, comfort with ambiguity, understanding other cultures, valuing diversity, and being able to recognize complex interconnections (Levy, Beechler, Taylor, \& Boyacigiller, 2007). As Keeling (2004) established in Learning Reconsidered, students do not simply absorb material presented to them; they need to be engaged with the material to integrate it into their personal development. Because cocurricular activities are based on personal engagement, they can therefore be essential to help students develop the skills necessary for global leadership.

Student Affairs is the name given to the group of services and the administration in higher education institutions that enhance student growth and development through cocurricular programs. One of the greatest challenges for this field is obtaining funding (Sandeen \& Barr, 2014), and as such creating a new program that specifically focuses on 
global leadership competencies may not be feasible for most institutions. However, many institutions have started to use the Student Leadership Competencies (SLC) to help identify learning outcomes for existing co-curricular programs. The SLCs are 60 leadership competencies derived from analyzing standards, models, and theories of leadership, and the outcomes of 522 accredited academic programs in higher education (Seemiller, 2013). Many institutions are publicizing the Student Leadership Competencies associated with each of their programs, so students can identify the competencies they wish to enhance and choose co-curricular activities that develop those competencies (Seemiller \& O'Keefe, 2016).

\section{Leadership}

There is no agreed upon research definition of leadership (Bass, 1990; Komives, Lucas, \&McMahon, 2007; Rost, 1993; Yukl, 2006). In fact, 60\% of the leadership research studies conducted from 1910 to 1990 did not offer a clear definition for leadership (Rost, 1993). The social scientists who did attempt definitions in their studies did not offer a holistic definition of leadership, but instead only defined the approach of the particular leadership they were studying (Komives et al., 2007; Yukl, 2006).

Yuki (2006) identified five general approaches to studying leadership: trait, behavior, situational, power-influence, and integrative. The trait approach studies the traits that make leaders different from their peers. The behavior approach focuses on what leaders actually do that is different from followers. The situational approach explores how the context and environment influences the effectiveness of a leader. The power-influence approach focuses on the processes that happen between a leader and 
his/her followers. Finally, the integrative approach involves studying combinations of the other four approaches (Bass, 1990; Komives et al., 2007).

Within each of these approaches there are leadership theories that have also been categorized by Yukl (2006): leader versus follower-centered theories, descriptive versus prescriptive theories, and universal versus contingency theories. Leader versus followercentered theories focus solely on the leader's behaviors and/or characteristics without much, if any, consideration of the followers (Bass, 1990). Descriptive versus prescriptive theories describe the typical activities of effective leaders. Universal versus contingency theories can be either prescriptive or descriptive ways that leadership issues are applied in different contexts (Yukl, 2006).

In general the goals of student affairs practitioners are to foster students' development in becoming self-aware and interpersonally sensitive individuals, democratic citizens, educated persons, skilled workers, and life skills managers (Hamrick et al., 2002). Student affairs practitioners use different leadership theories to assist in the design of programs that help to develop students' leadership potential. They accomplish this through leadership seminars/workshops, mentoring programs, guest speakers, service projects, outdoor education, and leadership courses (Zimmerman-Oster \& Burkhardt, 2000). As new leadership theories emerge, they try to incorporate them into existing programs or create new programs to help prepare students.

\section{Global Leadership}

The acceleration of globalization has created a need for an additional skill set not covered in the traditional leadership theories. The skill set is called global leadership (Blaess, Hollywood, \& Grant, 2012; Jokinen, 2005; Mendenhall \& Osland, 2002; J. S. 
Osland, Bird, Mendenhall, \& A. Osland, 2006; Rhinesmith, 1993). As with leadership, there is no agreed upon definition of global leadership. When trying to define global leadership, researchers focus on tasks and responsibilities, process, or both (Mendenhall, Reiche, Bird, and Osland, 2012). Suutari's (2002) definition is an example of tasks and responsibilities: "Global leaders are managers with global integration responsibilities in global organizations" (p. 229). An example of a process definition is "global leadership is the process of influencing the thinking, attitudes, and behaviors of a global community to work together synergistically toward a common vision and common goal" (Osland \& Bird, 2005, p. 123). A definition that includes both is "global leaders, defined as executives who are in jobs with some international scope, must effectively manage through the complex, changing, and often ambiguous global environment" (Caligiuri, 2006, p. 219).

Some of the new challenges for global leaders include, but are not limited to: working in different cultures; combining business practices to fit multinational needs; cultivating trust among team members that may not be of the same nationality or only work with each other remotely or both; overcoming communication barriers; creating clarity in team objectives where values may differ, dealing with different laws and regulations; overcoming stereotypes and prejudices; and managing through the complex, changing, and often ambiguous global environment (Caligiuri, 2006; Danielsson, 2015; Govindarajan \& Gupta, 2001; Hassanzadeh, Silong, Asmuni, \& Wahat, 2015; Holt, 2015; Levy et al., 2007; Taneja, Sewell, \& Odom, 2015; Voronchenko, Klimenko, \& Kostina, 2015). The Center for Creative Leadership handbook stated, "it is our contention that managers who fail to adapt how they lead, negotiate, make decisions, or share 
information to fit the global context are more likely to fail" (McCauley \& Van Velsor, 2004, p. 371). It has been argued that global leadership

...differs from domestic leadership in degree in terms of issues related to connectedness, boundary spanning, complexity, ethical challenges, dealing with tensions and paradoxes, pattern recognition, and building learning environments, teams and community and leading large-scale change efforts - across diverse cultures. (Osland \& Bird, 2006, p. 123)

For the purposes of this study, the researcher has used the definition of global leader of Mendenhall et al.:

global leaders are individuals who effect significant positive change in organizations by building communities through the development of trust and the arrangement of organizational structures and processes in a context involving multiple cross-boundary stakeholders, multiple sources of external crossboundary authority, and multiple cultures under conditions of temporal, geographical, and cultural complexity. (2013, p. 262)

As well as NACE's definition for the Global/Intercultural Fluency competency as "Value, respect, and learn from diverse cultures, races, ages, genders, sexual orientations, and religions. The individual demonstrates, openness, inclusiveness, sensitivity, and the ability to interact respectfully with all people and understand individuals' differences" (NACE, 2017).

\section{Global}

Mendenhall et al. (2012) discussed three dimensions of the global in global leadership - complexity, flow, and presence. Complexity is the contextual dimension. 
Lane, Maznevski, Mendenhall, and McNett, (2009) described four dimensions of complexity causing challenges in the face of globalization: multiplicity, interdependence, ambiguity, and flux. Multiplicity is the necessity of working with more and different competitors. Interdependence is managing complex and connected systems of human and technology interactions. Ambiguity is functioning with a lack of information. These three elements working together create the fourth, flux, which is the always changing element in explaining the complexities brought by an acceleration of globalization (Lane et al., 2009).

Flow is the relational dimension of global. There are two dimensions contained within flow: richness and quantity. Richness is "frequency of information flow, the volume of information flow, and the scope of information flow....Quantity refers to the magnitude or number of channels the global leaders must use to proactively boundary span in his/her role" (Mendenhall et al., 2012, p. 498). Technology allows resources and information to span across borders creating a global market instead of separate national markets (Caligiuri, 2006; Chase-Dunn, 1999; Govindarajan \& Gupta, 2001; Taneja et al., 2015). Beechler et al. (2004, p. 124) explained that effective individual global leaders "ensure that boundaries do not hamper the flow of essential knowledge and information. Interpersonal networks are vital in this effort because they serve as the glue that holds these vast geographically dispersed and internally differentiated organizations together."

Presence is the spatial-temporal dimension. Presence is how much time a person must physically be present with key stakeholders around the world in order to engage them. A low degree of presence is when there is little to no travel requirements. A medium level of presence is when occasional trips need to be made to other countries to 
visit stakeholders. A high degree is constant international business travel and to various locations (Mendenhall et al., 2012).

Being able to understand and work in a global environment where complexity, flow, and presence exist, and to be effective, requires a different set of leadership competencies. Identifying these specific leadership competencies is essential for, those persons who need to train and prepare others to successfully function in this type of environment and to help those desiring global positions educate themselves and enhance their skills in leadership.

\section{Student Leadership Competencies}

In 2008, researchers from the University of Arizona conducted a study that analyzed the Council for the Advancement of Standards (CAS) and identified outcomes related to leadership development, then created a framework of leadership competencies (Seemiller, 2016). They then used that framework to analyze contemporary leadership models: Relational Leadership Model, the Social Change Model of Leadership Development, and the Five Practices of Exemplary Leadership. After piloting this version of the framework in their programs, they assessed learning outcomes from academic programs and accrediting agencies. During the five year study, they analyzed learning outcomes in 413 academic programs from 49 different academic accrediting organizations affiliated with the Council for Higher Education Accreditation.

Subsequently they added 23 additional accrediting organizations from the Association of Specialized and Professional Accreditors and the U.S. Department of Education.

The result was identification of 60 competencies derived from the 72 different academic accrediting agencies was the result (Seemiller, 2013). After identifying these 
competencies which included such things as problem solving, self-development, group development, initiative, diversity, organization, conflict negotiation, and collaboration, Seemiller and Murray (2013) then conducted a study to statistically confirm the prevalence and frequency of the 60 competencies that existed among the 475 academic programs in the 72 accrediting organizations studied. These competencies are now being used by universities across the United States to help connect out-of-classroom activities to learning outcomes that resonate across all academic disciplines. Institutions are now redesigning current or creating new leadership programs based on these competencies. These programs range from workshops to semester long curricula (Seemiller, 2016). As institutions develop or adapt programs utilizing the Student Leadership Competencies, the competencies on which they focus are then specified in the various program learning outcomes. Different frameworks - academic, learning, retention, leadership, career, service, or professional, containing competencies derived from theory or research, are used to design programs and establish learning outcomes. These different frameworks generally focus on developing a smaller number of competencies because mastering 60 Student Leadership Competencies at one time is relatively unattainable. As well students may desire a different theory/research based framework than is being offered by their institution, so these resources are also being provided to students. The combination of advertising the learning outcomes for programs and utilizing frameworks enables students to create their own self-development plans (Seemiller \& O’Keefe, 2016). 


\section{Self-Assessments to Create Self-Awareness}

Becoming a self-aware, interpersonally sensitive individual, as well as an educated person is the focus in leadership education (Gehrke, 2006; Komives, Dugan, Owen, Slack, \& Wagner, 2011). A study of leadership identity development identified six stages of a student's experience: awareness, exploration/engagement, leader identified, leadership differentiated, generativity, and integration/synthesis (Komives et al., 2007, p. 395). Self-assessments are often used as the first step in creating selfawareness because they encourage students to begin to understand themselves by uncovering both strengths and; weaknesses that may have been unknown to them (Luft \& Ingham, 1961; Shertzer \& Doyle, 2006).

Assessments like the Meyers-Briggs Typology Indicator (MBTI; The Myers \& Briggs Foundation, 2016), StrengthsQuest (Gallup, 2016), Kolb Learning Style Inventory (LSI; McLeod, 2013), Leadership Practices Inventory (LPI; Wiley, 2016b), Personal Profile System (DiSC; Wiley, 2016a), Socially Responsible Leadership Scale (SRLS; National Clearinghouse for Leadership Programs, 2016), and True Colors (TC; True Colors Inc, 2016) are often used in leadership development programs not because they are great predictors of leadership ability (John \& Robins, 1993), but because their results facilitate self-reflection. Self-reflection then leads to better self-awareness, which allows people to better employ their strengths and improve their weaknesses (Drucker, 2005; Moore, Jenkins, Dietz, \& Feuerbaum, 1997; Luft \& Ingham, 1961; Pearman, 1999; Shertzer \& Doyle, 2006; Tjan, 2012; Travers, Morisano, \& Locke, 2015; ZimmermanOster \& Burkhardt, 2000). The Center for Creative Leadership claimed that assessments motivate because they create a "desire to close the gap between current self and ideal 
self" and bring "clarity about needed changes; clues about how the gap can be closed" (McCauley \& Van Velsor, 2004, p. 5).

Many of these assessments have associated developmental activities so as to give students the opportunity to further improve areas of weakness. As an example, the LPI gives results that refer to The Five Practices of Exemplary Leadership, a commonly used framework for emerging leadership programs (Kouzes \& Posner, 2016). When students take that inventory, they may identify that they need further development in Modeling the Way; and can then sign up for their institution's emerging leaders program to help develop that practice.

\section{Research Problem}

As the need for global leaders increases, employers are experiencing a skill crisis in that graduates are entering the workforce without the appropriate skills to perform in a global environment (Bersin, 2012; Elmore, 2013; Ficsher, 2015; Gillis, 2011; Zenger, Folkman, \& Evans, 2014). At the 2015 annual conference of NAFSA: Association of International Educators, Fanta Aw, NAFSA's president, stated that the demand for global competencies from graduates is high; we can no longer count on study abroad alone to prepare globally-minded students, and we need to be doing more at home to help them develop these skills (Fischer, 2015). Many universities have responded to the need for increasing global leadership skills by internationalizing the curriculum (ACE, 2013; Gacel-Ávila, 2005; Gibson, Rimmington, \& Landwehr-Brown, 2008; Global Learning, 2015; Grudzinski-Hall, 2007; Landorf \& Doscher, 2015; NAFSA, 2016).

Internationalizing the co-curriculum is also often considered part of this effort, though more could be done in this realm of the student's out-of-the-classroom education to help 
develop global leadership competencies (Commission on International Education, 1998; Ficsher, 2015; NAFSA, 2016).

Mendenhall (2006) stated, "people cannot develop global leadership competencies if they do not understand the degree to which they already possess these competencies" (p. 424). Specifically, there is a need for a global leadership competency assessment that can be used by student affairs practitioners to help students begin to understand their level of proficiency of global leadership competencies earlier in their development instead of once they enter the workforce (Zenger, 2014).

There are assessments currently available in the areas of global leadership but they have been developed for and by corporations as training and promotional tools for their top managers (Bird \& Stevens, 2013). Assessments developed by corporations are for their own particular global leadership assessments and models derived from the needs or observations of their employees in the context of the different countries in which they operate (Mendenhall et al., 2013). Some of these corporations have generalized global leadership assessments or created new entities to use the information they gained about global leadership competencies and market them to others. Such corporations include: Vangent; Global Leadership Excellence, LLC; Kozai Group, Inc; IDI, LLC; Van der Maesen Personnel Management; PAR, Inc; Thunderbird Schools of Global Management; Pfeiffer; and Aperian Global (Bird \& Stevens, 2013). While these global leadership assessments are useful for corporations, they were not designed to help provide selfawareness to college students. There are also no developmental activities associated with the results that are produced after taking the assessments. 
According to a comprehensive review of global leadership assessments conducted by Bird and Stevens (2013), there are several assessments designed for both education and/or business that have demonstrated reasonable validity and reliability measures. Of these the Global Mindset Inventory (GMI) and the Global Competencies Inventory (GCI) emerged as the most relevant for comparison against the objectives of the current study since they both take a more holistic approach to assessing global leadership. The GMI was designed for education and the GCI was designed for intercultural settings or crossculture encounters, whereas the other assessments were designed specifically for global leaders and expatriates in corporations (pp. 113-140).

The price, certification requirements, length of survey, and lack of developmental activities associated with the results of the current global leadership assessments are not conducive to their use by student affairs practitioners in leadership programs. The GMI costs about $\$ 150$ per individual user, requires the administrator to be certified, and takes about 15 minutes to complete. The GCI costs about $\$ 130$ per individual user, requires the administrator to be certified, and takes about 45 minutes to complete (Bird \& Stevens, 2013, p. 139).

There is a need for a global leadership competency assessment that is compatible with terminology currently being used by leadership development programs in university settings such as that used in the Student Leadership Competencies. Universities provide students with the opportunity to develop their leadership skills, but the emerging area of concentration - global leadership currently lacks appropriate resources and tools, such as a global leadership competency self-assessment instrument mapped within the Student Leadership Competencies. 


\section{Purpose of the Study}

The purpose of this study was to create a global leadership competencies selfassessment instrument mapped within the Student Leadership Competencies, and then demonstrate the extent to which the instrument yields evidence that supports valid and reliable inferences about students' global leadership competencies.

\section{Research Questions}

This study will address the following research questions:

1. Does the Global Leadership Competencies self-assessment instrument yield valid inferences about students' global leadership competencies?

2. Does the Global Leadership Competencies self-assessment instrument yield reliable inferences about students' global leadership competencies?

\section{Theoretical Framework}

Constructivism is the guiding theoretical framework of this study. Constructivism is grounded on the idea that human beings generate knowledge and meaning by reflecting on experience (Basseches, 1986; Baxter Magolda, 1992; Belenky, Clinchy, Goldberger, \& Tarule, 1997; Fisher, Rooke, \& Torbert, 2000; Kegan, 1982, 1994; Kitchener, 1986; Perry, 1968; Piaget, 1950). Kegan (1994) described continual adaptation as striving for the self-transforming mind or fifth order. The fifth order is the final level of consciousness where individuals see beyond themselves, others, and the systems of which they are a part, to form an understanding of how all people and systems interconnect. At the fifth level, humans are not just relying on their own thinking, but also relating to others while forming their own journey through life. Humans are constantly interpreting information presented to them then making decisions about to 
how to enhance themselves since it is in their genetic make up to develop intellectually (Piaget, 1950).

As people strive for the fifth order, they desire to be more self-aware because it helps them make sense of their journey. The instrument will ultimately be a tool that can be used in this process. Constructivism will help inform the analysis of the cognitive interviews where the researcher will be looking to identify and then address any elements

of the questions that could be contributing to response error (Willis, 2005). Specifically, interviewees will have different experiences they will reflect on as they answer the questions, so a similar understanding of what the question is asking will help decrease the response error. Additionally, it provides guidance in the analysis of the global leadership competencies literature since individuals will need to be functioning at the level of the fifth order to be a global leader.

\section{Significance of Study}

Currently, there are no appropriate tools for the university setting to help students develop global leadership competencies. Students, student affairs practitioners, and employers will all potentially benefit from the development of this instrument. Students will benefit from the self-reflection they may gain by utilizing the instrument. Student affairs practitioners will benefit by having a tool to help students develop global leadership competencies. Finally, employers will benefit because more students will graduate with global leadership competency skills. 


\section{Delimitations}

In this study, the sample for the cognitive interviews and pilot study was delimited to FIU students. The final sample was delimited to students who are enrolled in U.S. institutions of higher education.

\section{Assumptions}

The underlying premise of this study is that global leadership is a substantively different process than traditional leadership. The study stems from the idea that selfassessments are a useful educational tool that facilitates self-awareness.

\section{Definitions of Terms}

Cognitive Interviews. When participants verbalize their thoughts while they answer a survey question, so the interviewer can determine the inferences being made about the questions in the instrument.

Competencies. "Leadership competencies are knowledge, values, abilities, and behaviors that contribute to someone successfully completing a role or task" (Seemiller, 2013).

Constructivism. An epistemology based on the idea that human beings generate knowledge and meaning by reflecting on experience.

Exploratory Factor Analysis. A statistical method used to uncover the underlying structure of a relatively large set of variables. EFA is a technique within factor analysis whose overarching goal is to identify the underlying relationships between measured variables. 
Exploratory sequential mixed methods design. A mixed methods procedure where qualitative data are collected in early phases of a study; then, after that data is analyzed it is used to develop the instrument that will be used for the following quantitative data phase(s).

Framework. An underlying structure of a concept created by translating the components and/or research findings of models, theories, concepts, or studies to the 60 Student Leadership Competencies (Seemiller, 2016).

Globalization. "Globalization is a process of interaction and integration among the people, companies, and governments of different nations, a process driven by international trade and investment and aided by information technology" (The Levin Institute, 2015).

Global Leader. "Individuals who effect significant positive change in organizations by building communities through the development of trust and the arrangement of organizational structures and processes in a context involving multiple cross-boundary stakeholders, multiple sources of external cross-boundary authority, and multiple cultures under conditions of temporal, geographical, and cultural complexity" (Reiche \& Mendenhall, 2013, p. 262).

Reliability. "refers to the accuracy or precision of a measurement procedure" (Thorndike \& Thorndike-Christ, 2010, p. 118).

Student Leadership Competencies. Sixty leadership competencies derived from a 5year research study, conducted by Corey Seemiller, analyzing learning outcomes in 522 accredited academic programs in higher education (Seemiller, 2016).

Subject Matter Expert. A person who is an authority in a particular area or topic. 
Table of Specifications. A two-way chart used to identify relevant content of the items, which describes the topics to be covered by an instrument and the number of items or point values that will be aligned with each topic or response.

Theoretical Framework. A rationale for the study that provides the reader an understanding of the researcher's perception of the connection to theory.

Validity. "An evaluative judgment of the degree to which empirical evidence and theoretical rationales support the adequacy and appropriateness of interpretations and actions based on test scores or other modes of assessment" (Messick, 1996, p. 1). Validity evidence based on internal structure. An analysis that "can indicate the degree to which the relationships among test items and test components conform to the construct on which the proposed test score interpretations are based" (AERA, 2014, p. 16).

Validity evidence based on response processes. The evidence of validity based on information about the test takers' cognitive processes (AERA, 2014, p. 15).

Validity evidence based on test content. The evidence of validity based on the relationship between the content of the test and what it is intended to measure (AERA, 2014, p. 14).

\section{Overview of Succeeding Chapters}

This dissertation consists of four additional chapters. Chapter 2 presents a review of related literature including an overview of the global leadership literature and global leadership competency frameworks and models. Chapter 2 continues with a review of instruments used to assess global leadership and the validity and reliability studies conducted for these instruments. Chapter 3 describes the methods used for the study. It 
reviews the study's research questions, relevant conceptions of validity and reliability, the research design, descriptions of the sample, data collection procedures, data analysis procedures to be utilized to address the previously outlined research questions, and limitations. The results of the study and data analysis are presented in Chapter 4. Finally, Chapter 5 summarizes the study and research findings, provides an analysis of the results as they relate to the relevant literature, and presents study limitations, implications for practice, and recommendations for future research. 


\section{CHAPTER II}

\section{REVIEW OF LITERATURE}

Chapter 2 begins with an overview of the global leadership literature and global leadership competency frameworks and models. An examination of the literature is necessary to help identify and define current relevant global leadership competencies. Following this discussion, Chapter 2 continues with a review of instruments that have been used to assess global leadership and an exploration of validity and reliability studies conducted for these instruments.

\section{The Evolution of Global Leadership Literature}

Literature regarding global leadership began to emerge in the 1990s as a response to a need to distinguish the differences in the roles of domestic leaders, global managers, and expatriates as the world became more connected by the internet and proliferate of air travel. Domestic leaders are those who work within their own country, global managers are those who manage around the world, and an expatriate is someone whom lives outside their country. In the literature there was an evolving recognition that global leadership is more complicated than domestic leadership and required a different set of competencies in order for leaders to be successful (Mendenhall et al., 2013, p. 21). The concepts found throughout this evolution were used in helping to develop the Global Leadership Competencies framework.

In 1992, Tichy and his colleagues coined the term "true globalists" as those with a global mindset, global leadership skills and behaviors, energy, skills and talent for global networking, ability to build effective teams, and as having global change agent skills. Rhinesmith (1993) defined the term global mindset as those whom seek context, view life 
as a balance of contradictions, value diversity and team work, focus on process, see change as an opportunity, and strive for openness to the unexpected.

Yeung and Ready (1995) conducted the first quantitative cross-national global leadership study using 1,200 managers from eight different nations to discover their perceived most important global leadership capabilities. The eight different nations included in the study were Australia, France, Germany, Italy, Japan, Korea, United Kingdom, and the United States. The researchers found six common leadership competencies in the managers shared among these countries. Those leadership competences included: (a) the ability to articulate a tangible vision, values, and strategy, (b) being a catalyst for strategic change, (c) cultural change, (d) ability to empower others, (e) possession of both results and (f) customer orientation (p. 542).

In 1997, Brake presented a model called the Global Leadership Triad. The three characteristics he used to create the triad were (a) relationship management, (b) business acumen, and (c) personal effectiveness. The center of the triad consisted of transformational self, which is the "drive toward meaning and purpose through activity strengthened by reflection, personal mind management, and openness to change" (p.44). Each characteristic contained components. Business acumen's components were depth of field (an ability to switch perspectives from global to local), entrepreneurial spirit, professional expertise, stakeholder orientation, and total organizational astuteness. Relationship management included change agentry, community building, conflict management and negotiation, cross-cultural communication, and influencing. Lastly, personal effectiveness contained accountability, curiosity and learning, improvisation, maturity, and thinking agility. 
In 1999, Black, Morrison, and Gregersen devised the Global Explorer Model which identified four general capabilities of a global leader. They interviewed over 130 senior line and human resource executives in fifty companies in Europe, North America, and Asia and then interviewed forty nominated global leaders from these firms. The characteristics that emerged were: (a) inquisitiveness, (b) embracing duality, (c) exhibiting character, and (d) demonstrating savvy. Inquisitiveness is the love of learning and being intrigued by diversity. Embracing duality is when uncertainty is viewed as invigorating and a natural part of global business. Exhibiting character is the ability to connect emotionally with people of different backgrounds and cultures and consistently demonstrate personal integrity in a world full of ethical conflicts. Demonstrating savvy is being both business and organizational savvy (p. xi - xii). Harvey and Novicevic (2004) published a conceptual article that argued that global assignments like expatriation developed four types of global leader capital: (a) human capital, (b) cultural capital, (c) social capital, and (d) political capital. Human capital was the skills and competencies leaders need to have based on expert and referent power in their organization. Cultural capital was acceptance and social inclusiveness due to having tacit knowledge of how the organization operates. Social capital was the standing and concurrent ability to draw on standing to accomplish tasks in an organization. Finally, political capital was the ability to use power or authority and gain the support of constituents in a socially effective way (p. 1,177).

Marshall Goldsmith and a team of researchers gathered information from focus groups with $28 \mathrm{CEOs,} \mathrm{various} \mathrm{focus} \mathrm{groups/dialogue} \mathrm{forums} \mathrm{with} \mathrm{current} \mathrm{and} \mathrm{future}$ global leaders, 73 surveys, and over 200 interviews with high-potential leaders nominated 
by 120 international organizations. They discovered 15 dimensions of global leadership:

(a) demonstrating integrity, (b) encouraging constructive dialogue, (c) creating a shared vision, (d) developing people, (e) building partnerships, (f) sharing leadership,

(g) empowering people, (h) thinking globally, (i) appreciating diversity, (j) developing technical savvy, (k) ensuring customer satisfaction, (l) maintaining a competitive advantage, (m) leading change, (n) achieving personal mastery, and (o) anticipating opportunities. They predicted that thinking globally, appreciating cultural diversity, developing technological savvy, building partnerships and alliances, and sharing leadership would be especially important in the future as globalization increased (Goldsmith et al., 2003, p. 329 - 333).

The literature attempted to distinguish and define domestic leaders, global managers, and expatriates. As the literature on global leadership developed, it identified the traits and competencies needed to be a global leader. Frameworks and models of global leadership characteristics started to emerge as resources for corporations and individuals desiring to learn or teach global leadership.

\section{Global Leadership Competency Frameworks and Models}

As global leadership competencies have been identified throughout the years, researchers have attempted to group them into useable frameworks and models. Many similar competencies appear in different frameworks and models, which demonstrated some consensus on individual competencies, although there is still no complete agreement on a useful organization of those competencies. The frameworks and models below are examples of some of the latest research that attempted to organize global leadership competencies. 
In 2002, Mendenhall and Osland observed the efforts being made by corporations to create global leaders and uncovered a multidimensional construct of global leadership. They discovered some conceptual patterns after reviewing 56 different competencies being used by these corporations. They categorized those into six core dimensions: (a) cross-cultural relationship skills, (b) traits and values, (c) global business expertise, (d) global organizing expertise, (e) cognitive orientation, and (f) visioning. Crosscultural relationship skills referred to developing and maintaining interpersonal relationships in global/cross-cultural contexts. Traits and values are personality traits and habits. Global business expertise is knowledge about the global business practices. Global organizing expertise is the ability to organize and structure processes in a global context. Cognitive orientation referred to how one processes information and their world-view. Finally, visioning is knowing where the company should be heading and knowing how to get others to help achieve that vision (Osland, 2013).

In 2004, Bird and Osland developed a pyramid model of global leadership. Their premise was that one must have the base skills in order to have or learn the upper level skills. The foundation for their pyramid was global knowledge. Level one is traits, which include integrity, humility, inquisitiveness, and hardiness. These were also referred to as the threshold traits. Level two was attitudes and orientations, which include cognitive complexity and cosmopolitanism. Level two was referred to as global mindset. Level three was interpersonal skills including mindful communication and creating and building trust. Finally, at the top, level four was systems skills, which included making ethical decisions, span boundaries, and building community through change (Osland, 2013). 
In 2005, Jokinen reviewed all previous global leadership and expatriate competency models to create a more integrated global leadership competency model. The model had three tiers: (a) core global leadership competencies, (b) desired mental characteristics of a global leader, and (c) behavioral level global competencies. Core global leadership competencies were self-awareness, engagement in personal transformation and inquisitiveness. To be self-aware indicated that a person is knowledgeable of their strengths, weaknesses, desires, motivators, and typical reactions. Self-awareness is important because it helped the person assess a situation and figure out how they can assist with it. Engagement in personal transformation was the idea of trying to keep oneself up-to-date, always looking for new opportunities to learn and enhance oneself as well as being open to change. Inquisitiveness, on the other hand, was curiosity and the desire to find new information about old topics. It often helped motivate a person to take risks, initiative, and commit to new projects.

According to Jokinen, desired mental characteristics of a global leader are "characteristics that affect the way a particular individual attempts to influence others and approaches a certain task" (p. 206). The characteristics included optimism, selfregulation, social judgment skills, empathy, motivation to work in an international environment, cognitive skills, and acceptance of complexity and its contradictions. Optimism was the idea that good will prevail. A person will be more motivated to do something that they believe will happen, so if a person is remaining positive, a can-do attitude emerges during complicated situations. Self-regulation was the ability to control your impulses and moods. Social judgment skills referred to the ability to look beyond the situation to the bigger picture; a systems approach that allows a person to switch 
viewpoints and understand interdependence. Empathy was having a genuine concern for others' needs and perceptions and helped one develop and embrace cross cultural sensitivity. Motivation to work in an international environment was important or a person will lose motivation quickly if they never wanted to work with an international population in the first place. Cognitive skills determined how much one learns from a situation or environment. These skills also helped an individual to create new solutions to problems and allow them to quickly switch their concentration from one thing to another based on need. Acceptance of complexity and its contradictions is essential for a leader that is dealing with a culture that is different from their own. Many of the situations a leader encountered may seem ambiguous and unpredictable, so one must see these conditions as opportunities to succeed (Jokinen, 2005).

Behavioral level global competencies are "related to abilities to perform concrete actions and producing visible results" (Jokinen, 2005, p. 208). The behavioral level global competencies entailed social skills, network management skills, and knowledge. Social skills are more informal person-to-person interactions. A leader must be a change catalyst, visionary, good at building and leading teams, have strong communication and listening skills, be motivating, and able to manage conflict. Essentially, one must be good at bringing out the best in people. Network management skills referred to formal relationships created through organizations. One must actively pursue partnerships, create networks, and build connections so they have a community with which to work. Knowledge refers to the technical stuff. They must understand who has the power and influence in their company and what processes and procedures are important. They must 
also clearly know how to do their specific job. Most importantly, they must also do the research on the culture with which they are trying to work (Jokinen, 2005).

Javidan and Walker (2013) consolidated the prior research on global mindset and created three categories: intellectual capital, psychological capital, and social capital, which are further defined by three competencies in each category. The intellectual capital competencies included global business savvy, cosmopolitan outlook, and cognitive complexity. The psychological capital competencies were passion for diversity, quest for adventure, and self-assurance. Finally, the competencies that make up social capital were intercultural empathy, interpersonal impact, and diplomacy.

Intellectual Capital was the cognitive category of global mindset. The first component, global business savvy, consists of knowledge of global industry, global competitive business and marketing strategies, how to transact business and assess risks of doing business internationally, and supplier options in other parts of the world. Global business savvy concept was not just for business people, but demonstrated the need to understand the processes of whatever industry the person is working for globally. The next component was a cosmopolitan outlook, which includes knowledge of cultures in different parts of the world, geography, history, and important persons of several countries, economic and political issues, concerns, and hot topics of major regions of the world, and important world events. Finally, cognitive complexity comprised the ability to grasp complex concepts quickly, analyze and problem-solve, understand abstract ideas, and take complex issues and explain the main points simply and understandably (Javidan \& Walker, 2013). 
Psychological Capital was the affective component of global mindset. Passion for diversity referred to enjoyment of exploring other parts of the world, getting to know people from other parts of the world, living in another country, and traveling. Quest for adventure discussed the interest in dealing with challenging situations, willingness to take risk and test one's abilities, and enjoyment of dealing with unpredictable situations. Finally, self-assurance was being energetic, self-confident, comfortable in uncomfortable situations, and witty in touch situations (Javidan \& Walker, 2013).

Global Social Capital was the behavioral component of global mindset. Intercultural empathy is the ability to work well with people from other parts of the world, understand nonverbal expressions of people from other cultures, emotionally connect to people from other cultures, and engage people from other parts of the world to work together. Interpersonal impact is experience in negotiating contract/agreements in other cultures, having strong networks with people from other cultures and with influential people, and your reputation as a leader. Diplomacy is ease of starting conversation with a stranger, ability to integrate diverse perspectives and listen to what other have to say, and willingness to collaborate (Javidan \& Walker, 2013).

In 2013, Bird reviewed theoretical and empirical studies published from 1993 to 2012 in an effort to organize global leadership competencies into a framework of nested global leadership competencies. He found 160 separate competencies associated with global leadership. He grouped these competencies into three categories: (a) business and organizational acumen, (b) managing people and relationships, and (c) managing self. Each of these categories contain composite competencies, which provide more specific 
skills, abilities, knowledge bases, or orientations. Fifteen complex, multifaceted global leadership competencies were created.

The first category, business and organizational acumen, was the "practical understanding or business and organizational realities and how to get things done efficiently and effectively" (Bird, 2013). It contained composite competencies of vision and strategic thinking, business savvy, organizational savvy, managing communities, and leading change. Vision and strategic thinking encompasses the ability to comprehend and strategically think about the complexity of the environment, activities related to developing and articulating a global vision, and the ability to develop and implement a global strategic plan. Business savvy includes practical understanding and wisdom, as well as, an attitude toward finding efficient value adding solutions. Managing communities focuses on the ability to deal with the network of relationships through boundary-spanning, influencing stakeholders, and cultivating a community of stakeholders that help accomplish strategic objectives. Organizational savvy is the ability to design global organizational structures and processes. Leading change is the ability to implement change (Bird, 2013, p. 89 - 90).

The second category, managing people and relationships was "directed toward people and relationships" (Bird, 2013). It contained the composite competencies of cross-cultural communication, interpersonal skills, valuing people, empowering others, and teaming skills. Cross-cultural communication includes cultural awareness of both self and others, as well as, the ability to communicate across cultures through speaking the language, negotiating, and contextualizing communication in culturally appropriate ways. Interpersonal skills are broken into the broad definitions of emotional intelligence 
and relationship management skills. Valuing people is the foundation of this category, which includes respecting differences, the ability to understand people as individuals, and creating and maintaining trusting relationships. Empowering others is energizing individuals by increasing their self-efficacy. And finally, teaming skills is the ability to work in multicultural and global virtual teams (Bird, 2013).

The third category, managing self, is "directed inward to the predispositional, cognitive, and attitudinal processes in the mind of the global leader or involve aspects of personal management" (Bird, 2013, p. 92). The third category contained the composite competencies of resilience, character, inquisitiveness, flexibility, and global mindset. Resilience is the ability to cope with the highly stressful situations and incorporates work-life balance and maintenance of physical, social, and mental health. Character is a combination of integrity, maturity, and conscientiousness. Inquisitiveness is an innate curiosity, being open-minded, having humility, and life-long learning. Flexibility is the willingness to tolerate ambiguity and adapt to various situations. Lastly, global mindset includes having cognitive complexity and cosmopolitanism (Bird, 2013).

The various frameworks and models discussed her demonstrate some consensus on individual competencies, though not completely. These models and frameworks have attempted to organize the competencies of a global leader for use by corporations and individuals in training and learning. However, in order to use them there needed to be an ability to assess or measure those competencies and their identified attributes.

\section{Global Leadership Assessments}

As the research on global leadership progressed, researchers sought to measure and attempt to predict this phenomenon. Bird and Stevens (2013) conducted a review of 
global leadership assessment instruments and classified them into three categories: cultural difference assessments, intercultural adaptability assessments, and global leadership competency assessments. Cultural difference assessments were not used to directly measure global leadership competencies, but some companies have used them for indirect competency assessment. Intercultural adaptability assessments were often associated with global manager development programs since interactions with different cultures is essential. Global leadership competency assessments attempted a broader focus that is beyond just intercultural competence (Bird \& Stevens, 2013). The literature review further divides and discusses global leadership competency assessments most appropriate for use in a business setting and an educational setting.

\section{Cultural Difference Assessments}

Cultural difference assessments are instruments used to help identify differences in national cultural values. In 2009, Taras, Rowney, and Steel conducted an analysis of 121 instruments for measuring culture. They found most instruments utilized selfassessments to try to quantify culture. Sample size and procedures of the instruments varied and over 60 countries/societies representing the majority of the world's population were surveyed. They concluded that the major challenges with cultural surveys were that data need to be collected from multiple cultures to provide a basis for comparison, the researcher must choose between sample representativeness and cross-sample comparability, and use items that are familiar across multiple cultures.

In regard to reliability, they found that the average Cronbach's alpha to be 0.67 with a range of 0.41 to 0.82 . When examining validity reported for these instruments, only $30 \%$ reported on the validity of the measure. Most reported out on criterion-related 
validity where they analyze the correlations between the test scores and theoretically relevant external variables. Convergent validity was also reported out for the large-scale surveys that compared the national cultural data to data from prior instruments.

The researchers concluded that while there were improvements to conceptualizing and measuring culture as instruments progressed, the most notable developments "go beyond studying culture exclusively" (Taras et al., 2009, p. 369). So, intercultural adaptability assessments and global leadership assessments have emerged. While cultural difference assessments might be useful in some situations, they only provide information on one aspect of global leadership. These assessments will not be used in this study's comparison for these reasons.

\section{Intercultural Adaptability Assessments}

Intercultural adaptability assessments are instruments that focus on intercultural competence. In 2013 Matsumoto and Hwang conducted an analysis of instruments that measure cross-cultural competence. The ten assessments included in their analysis were Cross-Cultural Adaptability Inventory (CCAI), Cross-Cultural Sensitivity Scale (CCSS), Cultural Intelligence Scale (CQ), Intercultural Behavioral Assessment (IBA), Behavioral Assessment Scale for Intercultural Communication Effectiveness (BASIC), Intercultural Adjustment Potential Scale (ICAPS), Intercultural Communication Competence (ICC), Cultural Intelligence Scale (ICSI), Intercultural Development Inventory (IDI), Intercultural Sensitivity Scale (ISS), and Multicultural Personality Inventory (MPQ). They chose instruments that had empirical articles published in English in peer reviewed journals. 
They examined content validity, construct validity, and ecological validity for each of the 10 instruments. The ICC and BASIC surveys had questionable content validity, while the other instruments demonstrated reasonable validity, though measured different knowledge, skill, and ability domains. Construct validity was lacking for all instruments except MPQ and CQ where multiple studies had been conducted to confirm the factors being measured in each of those assessments. Additionally, it was found that the evidence for ecological validity was also inadequate for all the instruments except CQ, ICAPS, and MPQ, because "they lack the use of valid and reliable criterion variables of intercultural adjustment or adaptation, have limited breadth of cross-cultural samples, do not use mixed methodologies, and do not provide evidence of concurrent, predictive, and/or incremental ecological validity" (Matsumoto \& Hwang, 2013, p. 866).

Matsumoto and Hwang (2013) concluded that CQ, ICAPS, and MPQ emerged as the instruments demonstrating the most validity in measuring intercultural adaptability of the ten instruments analyzed. The domains measured in these three instruments will be included when forming the Global Leadership Competencies framework. The CQ domains included motivational $\mathrm{CQ}$, cognitive $\mathrm{CQ}$, metacognitive $\mathrm{CQ}$, and behavioral $\mathrm{CQ}$. The ICAPS domains include emotion regulation - emotional robustness, openness rigidity, flexibility and creativity, critical thinking and social conscientiousness. The MPQ domains included cultural empathy, open-mindedness, social initiative, emotional stability, and flexibility (Matsumoto \& Hwang, 2013). There are two other instruments that fall into the intercultural adaptability assessment category, not included in the study above but relevant to this study: Global Competence Aptitude Assessment and Global Perspectives Inventory. 
The Global Competence Aptitude Assessment (GCAA) was developed by Hunter, White, and Godbey (2006). There are two different domains: Internal Readiness and External Readiness. Each domain contained four components. Internal Readiness includes being self-aware, willing to take risks, being open-minded, being perceptive, and respectful of diversity. External Readiness consists of globally aware, knowledgeable about world history, interculturally competent, and effective across cultures. The GCAA is recognized by the American Council on Education (Global Leadership Excellence, LLC., 2016), so considering these domains when developing the Global Leadership Competencies framework will be important.

The Global Perspectives Inventory was developed under the direction of Larry Braskamp and contains three domains: Cognitive, Intrapersonal, and Interpersonal. Each domain contains two components. Cognitive includes knowing and knowledge. Intrapersonal comprises of identity and affect. Finally, Interpersonal consists of social responsibility and social interactions. The instrument has been being refined since 2007 to increase the estimates of both reliability and validity (L. Braskamp, D. Braskamp, Engberg, 2014). The Global Perspectives Inventory is also being used by several universities and will be important to include the domains when developing the Global Leadership Competencies framework.

\section{Global Leadership Competencies: Corporate}

The assessments described above focus on measuring cultural or international competence and/or adaptability, whereas the instruments included in this section measure a broader range of competencies. While cultural competence is important for global leadership, there are other specific leadership related competencies necessary to be 
successful in a global environment that are not measured by the instruments described above. The instruments included in this section contain these extended competencies, although are focused for use in a corporate setting. Because they are most important for corporate business, they may not be as necessary for all student programs but they have generalizability so can be used in an educational setting.

Global Executive Leadership Inventory. The instrument was designed from research done with executives involved in training programs at INSEAD, a global graduate business school. Twelve dimensions emerged in the research: (a) visioning, (b) empowering, (c) energizing, (d) designing and aligning, (e) rewarding and feedback, (f) team building, (g) outside orientation, (h) global mindset, (i) tenacity, (j) emotional intelligence, (k) life balance, and (l) resilience to stress. The instrument is a 360-degree feedback assessment that requires two observers such as a supervisor, coworker, direct report, or acquaintance to complete an observer's version of the instrument and the leader to complete a leader's version of the instrument (Kets de Vries, Vrignaud, \& FlorentTreacy, 2004).

GlobeSmart Leadership Assessment (GLA). The instrument was developed through research at Aperian Global (2016) and is used primarily in business settings. It measures 5 domains: (a) seeing differences, (b) closing gaps, (c) opening the system, (d) preserving balance, and (e) establishing solutions. It is also a 360-degree assessment to be used on individuals already in global leadership roles.

\section{Global Leadership Competencies: Education}

The Global Mindset Inventory and Global Competencies Inventory emerged as two of the top global leadership competency assessments most appropriate for education. 
They were designed with being used in an educational setting in mind. They are also more generalizable than the corporate global leadership assessments.

Global Mindset Inventory. The Global Mindset Inventory measures 3 categories and their facets in a 76-question survey. The conceptual basis for the inventory's categories and dimensions was derived from a combination of responses from over 1,000 global executives and input from a group of academicians. Respondents use self-evaluation on a five-point scale to express the degree they believe they possess each competency. There is a GMI certification needed in order to administer the assessment, costs $\$ 150$, and takes about 15 minutes to complete (Bird \& Stevens, 2013, p. 128 -139).

The researchers divided global mindset into three categories: intellectual capital, psychological capital, and social capital each of which have three competencies. The intellectual capital competencies included global business savvy, cosmopolitan outlook, and cognitive complexity. The psychological capital competencies were passion for diversity, quest for adventure, and self-assurance. Finally, the competencies that make up social capital were intercultural empathy, interpersonal impact, and diplomacy.

Intellectual capital was the cognitive component of global mindset. "It is your knowledge of and ability to understand international business, business processes, and the cultural underpinnings of multiple countries around the globe" (Javidan \& Walker, 2013, p. 17). The first component, global business savvy, consisted of knowledge of global industry, global competitive business and marketing strategies, how to transact business and assess risks of doing business internationally, and supplier options in other parts of the world. The next component was cosmopolitan outlook, which includes knowledge of cultures in different parts of the world, geography, history, and important persons of 
several countries, economic and political issues, concerns, and hot topics of major regions of the world, and important world events. Finally, cognitive complexity comprised the ability to grasp complex concepts quickly, analyze and problem-solve, understand abstract ideas, and take complex issues and explain the main points simply and understandably.

Psychological capital was the affective component of global mindset. "It refers to your motives and values, and it reflects your willingness and motivation to experience and to succeed in international settings" (Javidan \& Walker, 2013, p. 18). The three components contained of this category were passion for diversity, quest for adventure, and self-assurance. Passion for diversity referred to enjoyment of exploring other parts of the world, getting to know people from other parts of the world, living in another country, and traveling. Quest for adventure encompassed interest in dealing with challenging situations, willingness to take risk and test one's abilities, and enjoyment of dealing with unpredictable situations. Finally, self-assurance was being energetic, self-confident, comfortable in uncomfortable situations, and witty in touch situations.

Global social capital was the behavioral component of global mindset. "It reflects your ability to interact appropriately in cultures around the world and affects your ability to build trusting relationships with individuals who are different from you" (Javidan \& Walker, 2013, p. 19). The three components included intercultural empathy, interpersonal impact, and diplomacy. Intercultural empathy was the ability to work well with people from other parts of the world, understand nonverbal expressions of people from other cultures, emotionally connect to people from other cultures, and engage people from other parts of the world to work together. Interpersonal impact was 
experience in negotiating contract/agreements in other cultures, having strong networks with people from other cultures and with influential people, and your reputation as a leader. Diplomacy was ease of starting conversation with a stranger, ability to integrate diverse perspectives and listen to what other have to say, and willingness to collaborate (Javidan \& Walker, 2013).

The Thunderbird Global Mindset Institute conducted a multiphase multimethod research methodology that resulted in strong reliability and validity for the Global Mindset Inventory instrument. The instrument began with 91 questions and through a three-phased confirmatory factor analysis, ended with 76 questions - 50 addressed global mindset and 26 collect demographic information. The researchers piloted the instrument with 1,266 participants, which consisted of both MBA students from the Thunderbird School of Global Management and global managers from two different undisclosed companies (Javidan et al., 2010).

They discovered the three categories (Psychological, Social, and Intellectual Capital) were not distinct from each other, but the nine components that make up the three categories were significant and distinct because the correlational range for the components were $r=.34-.67$, which is moderate (Hu \& Bentler, 1999). They confirmed the significance of the nine components by running a second confirmatory factor analysis with a larger group, which resulted in a Chi-Square $=10110.52(d f=1139)$. Exploratory factor analysis of the component scores were used to determine internal consistency. The Cronbach's alpha reliability was determined to be over .70, which is strong, for all components separately in both the student and executive samples. Therefore, the three 
categories are discussed in a theoretical sense and the nine components were used as the actual indicators of global mindset (Javidan et al., 2010).

To support validity, they ran univariate analysis using the demographic information collected and compared it to leadership and cross-cultural theory and research. They examined effect on demographic information for: individual's level within the organization, size of the organization, education level, age, gender, individual obtainment of an international degree, English proficiency, number of languages spoken, number of countries lived in and length of stay, number of friends and family-friends from other countries, and board of director officer positions held. Criterion-related validity tests were also conducted to determine the instruments ability to predict top performance in global leaders. The two companies that participated in the study submitted performance related information, which was compared to the global mindset inventory results for those employees. The data provided by both companies were significant in validating criterion (Javidan et al., 2010).

The strengths of the Global Mindset Inventory are that it has shown high reliability and content validity and it has moderate predictive and face validity. Additionally, it was designed for use in education and only takes about fifteen minutes to complete. The weaknesses of the Global Mindset Inventory are that requires certification to administer it, it is moderately complex to use, and it is relatively expensive at $\$ 150$ per administration.

Global Competencies Inventory. Bird, Stevens, Mendenhall, and Oddou initially developed the Global Competencies Inventory in 2000 from an elaboration of an expatriate adjustment model. It measured seventeen dimensions that are grouped into 
three factors; perception management, relationship management, and self-management. Respondents to the 180-question survey use self-evaluation on a five-point scale to express the degree they believe they possess each competency. Certification is required to administer the assessment, it costs $\$ 130$, and takes about 45 minutes to complete (Bird \& Stevens, 2013).

The first of the three factors, perception management, addresses how people perceive differences, their ability to be flexible and manage those perceptions, as well as, their curiosity toward differences. The dimensions of that factor include nonjudgementalness, inquisitiveness, tolerance of ambiguity, cosmopolitanism, and interest flexibility. Nonjudgmentalness is the extent to which one is able to avoid quick judgments regarding unfamiliar people, situations, or behaviors. Inquisitiveness is the openness towards, and an active pursuit of understanding, ideas, values, norms, situations, and behaviors that are new and different. Tolerance of ambiguity reflects an ability to cope with uncertainty in new and complex situations. Cosmopolitanism refers to an interest in different countries and cultures, as well as an interest in world and international events. Interest flexibility is being willing to embrace interests different than their own in the host culture (Bird \& Stevens, 2013).

The second factor, relationship management is the ability to develop and maintain relationships through awareness of themselves and others. The dimensions are relationship interest, interpersonal engagement, emotional sensitivity, self-awareness, and behavior flexibility. Relationship interest is the degree someone is interested and aware of their social environment. Interpersonal engagement is the extent to which someone is able and willing to initiate and maintain relationships with other from a different culture. 
Emotional sensitivity is how much a person is aware of and sensitive to the feelings of others. Self-awareness is the extent to which people possess awareness of themselves in their interactions with others. Behavioral flexibility is adjusting and presenting oneself in a favorable impression to help build constructive relationships (Bird \& Stevens, 2013).

The third factor is self-management, which incorporates self-identity and being able to adapt while remaining mentally and emotionally healthy. The dimensions included are optimism, self-confidence, self-identity, emotional resilience, non-stress tendency, and stress management. Optimism ability to stay positive and see challenges as learning opportunities. Self-confidence refers to the trust and assurance people have in themselves, and to the inclination to believe that through persistence they can overcome obstacles. Self-identity is being able to maintain personal values no matter what situational factors, as well as, a sense of personal identity. Emotional resilience reflects the degree to which a person possesses the emotional strength and resilience to cope with stressful and challenging intercultural situation. Non-stress tendency reflects the scope of the dysfunctional stressors that may influence people in their daily work and social life in intercultural situations. Stress management is the extent to which individuals actively employ various techniques or practices to cope with and recover from stress and also the degree to which they effectively organize their time (Bird \& Stevens, 2013).

The Kozai Group conducted a study to prove reliability, convergence with other similar inventories, and predictability on the Global Competencies Inventory instrument. The pilot study included 2,308 subjects found through both random and convenience sampling to get diverse subjects in profession, education, ethic, and demographic groups. The instrument started with 327 items and ended with 171 items after running principle 
component analysis to discover the items that best represented the 16 subscales. The 171 items that remained showed factor loadings at least greater than .4, and subscale alphas were all above .72, which indicated strong reliability (Stevens et al., 2014). They also ran differential validity to be sure there was no test bias with 21 different subgroups. They found that while there were some differences in the subgroups, the individual score was more reflective of the person than the overall subgroups differences (Stevens, Bird, Mendenhall, \& Oddou, 2014).

To create construct validity, the researchers in the Korzai Group ran convergent validity against NEO PI-R as it assessed a five-factor model of personality with six distinct subfacets in each, which is closely related to the GCI in both format and constructs. There were several studies conducted to confirm internal consistency, reliability and validity across different populations. Four subject matter experts were recruited to independently determine where there was convergence between the two scales. At least three of four predicted convergence for 95 of the intercorrelations. This was later confirmed through administering both assessments to 179 graduate and undergraduate students from four different universities, where 91 of the 95 had a correlation of at least $r=.42$ indicating convergent validity between the two instruments (Stevens et al., 2014).

Three studies were conducted to prove criterion related validity. The GCI was used with 305 Japanese managers while on expatriate work assignments. This study found "predictive validity of GCI for global management competencies learning while on the overseas assignment $(r=.53)$, global management competencies transfer upon repatriation $(r=.42)$, and self-reports of job motivation upon repatriation $(r=.45)$ " (p.137). 
Another study conducted with Marines found that the higher their GCI score, the higher level of repatriate adjustment. The final study used a group of Japanese college students and found that the GCI was a strong predictor for foreign language acquisition (Stevens et al., 2014).

The strengths of the Global Competencies Inventory are that it is high in reliability, as well as, content, convergent, and face validity. It also shows moderate predictive validity, has tested to have no differential bias, and does a social desirability check. The weaknesses of the Global Competencies Inventory are that it requires a certification to administer it, costs $\$ 130$, takes 45 minutes to complete, and is meant for intercultural settings or cross-cultural encounters not in education.

\section{Summary}

The literature review established the theoretical development of the current relevant global leadership competencies. The literature review also demonstrated that the current assessments for global leadership were developed for the corporate setting and are not as useful in the university setting. However, as a result of the review of development, reliability, and validity data provided by the instruments that were designed for corporations, I was able to identify methods that could provide useful data for this study. These methods will be described in detail in Chapter 3. 


\section{CHAPTER III}

\section{METHODS}

Chapter 1 served as an introduction to this investigation, presenting information concerning the study's problem, purpose, research issues, and relevant theoretical influences. Chapter 2 reviewed the pertinent research. Chapter 3 reviews the methods that were used in this study. It includes the research questions, the relevant aspects of validity and reliability, and the research design, descriptions of the samples, data collection procedures, and data analysis procedures.

\section{Research Questions}

The study aimed to develop and validate a self-assessment instrument of global leadership competencies and addressed two following research questions:

1. Does the Global Leadership Competencies self-assessment instrument yield valid inferences about students' global leadership competencies?

2. Does the Global Leadership Competencies self-assessment instrument yield reliable inferences about students' global leadership competencies?

\section{Research Design}

The researcher used an exploratory sequential mixed methods design, which is a mixed methods procedure where qualitative data are collected in early phases of a study; then, findings from the qualitative data are used for the following quantitative data phase(s) (Teddlie \& Tashakkori, 2006). A meta-interpretation, which is a holistic interpretive synthesis of the results, is inferred at the end derived from the findings from both phases (I. Newman, D. Newman, C. Newman, 2011). Mixed methods were chosen for this study because the "qualitative-quantitative procedures need to be carried out in a 
manner that has credibility and will inform the results produced by the other, and not in isolation from each other" (I. Newman et al., p. 196). Mixed methods research also provides the strengths of both quantitative and qualitative research while allowing the researcher to generate or test a theory and "provide stronger evidence for a conclusion through convergence and corroboration of findings" (Johnson \& Onwuegbuzie, 2004, p. 21).

Exploratory sequential design is often referred to as the instrument development design. The separate phases of this design make it easier to explain, implement, and report. Though the emphasis is often on the qualitative phases, the quantitative phases are better received by some audiences, so using both makes the research findings more acceptable. Finally, one of the main strengths of this design is being able to "produce a new instrument" (Creswell \& Plano Clark, 2011, p. 106). In the current study, the qualitative data collected through the feedback of the experts and the cognitive interviews with students in the early stages of the study was the foundation for creating and refining the items on the instrument. Inferences were made from the results of analyzing the quantitative data collected from the instrument as well as from interpreting the feedback from the qualitative data of those two groups.

The process used in designing a survey in this study began with defining the research objectives, conducting a literature review and consulting experts' opinions to develop items. The items were then subjected to an expert review, which happened while choosing the mode of collection and choosing a sampling frame. Cognitive pretesting was conducted before executing a pilot test while simultaneously designing and selecting a sample. The next steps were to recruit and measure the sample, code and edit the data, 
make post survey adjustments, and perform an analysis (Gehlbach \& Brinkworth, 2011; Groves et al., 2011).

\section{Population}

The population for the study was undergraduate students enrolled in institutions of higher education across the United States of America (USA).

\section{Relevant Conceptions of Validity and Reliability}

According to the Standards of Educational and Psychological Testing, validity "is the degree to which all the accumulated evidence supports the intended interpretation of test scores for the proposed use" (AERA, 2014, p. 14). The focus of what exactly is validated has evolved over time; it began with the test itself, then focused was on the test in a certain setting with the emphasis on the ability to predict behavior, and now the Standards suggests that it is the scores of the individuals and the inferences and interpretations researchers make from those scores (Hubley \& Zumbo, 1996). Since this evolution, we are now not only attempting to validate the instrument, but also "the theory behind the inferences made of the test scores" (Hubley \& Zumbo, 1996, p. 212).

The Standards of Educational and Psychological Testing identify evidence sources that highlight different aspects of validity: test content, response processes, internal structure, and relations to other variables. Evidence based on test content is the relationship between the content of the test and what it is intended to measure. Test content refers to "themes, wording, and format of the items, tasks, or questions on a test" (AERA, 2014, p. 14). Evidence based on response processes are essentially the cognitive processes engaged in by test takers. This "can provide evidence concerning the fit between the construct and the detailed nature of the performance or response actually 
engaged in by test takers" (AERA, 2014, p. 15). Evidence based on internal structure is an analysis that "can indicate the degree to which the relationships among test items and test components conform to the construct on which the proposed test score interpretations are based" (AERA, 2014, p. 16). Evidence based on relations to other variables is when the "intended interpretation for a given use implies that the construct should be related to some other variables, and, as a result, analyses of the relationship of test scores to variables external to the test provide another important source of validity evidence" (AERA, 2014, p. 16).

The evidence for validity needed depends on the "proposition that underlies a proposed test interpretation for a specific use" (AERA, 2014, p. 14). Messick (1989), who provided the basis on which the current standards were mainly developed, also believed that validity was not "all or none" (p. 13) and that it is actually a process, explained as "constructing and evaluating arguments for and against the intended interpretation of the test scores and their relevance to the proposed use" (AERA, 2014, p. 11). Thus, validation is "essentially a matter of making the most reasonable case to guide both current use of the test and current research to advance understanding of what the test scores mean" (Messick, 1989, p. 13).

Reliability is the "consistency of scores across replications of a testing procedure" (AERA, 2014, p. 33). There are three types of reliability: stability, consistency, and dependability. Stability describes the instrument's susceptibility to extraneous factors from one administration to the next. Dependability describes the ability of the instrument to behave predictably each time it is used (Thorndike \& Thorndike-Christ, 2010). Internal-consistency estimates of reliability, such as Cronbach's coefficient alpha, are 
"based on the relationships/interactions among scores derived from individual items or subsets of items within a test, all data accruing from a single administration" (AERA, 2014, p. 37). Cronbach's coefficient alpha can also determine if a set of items are unidimensional and describes the extent to which the items measure a construct on an instrument (Tavakol \& Dennick, 2011).

\section{Data Collection Procedures and Methods}

The intended audience for the interpretation of the instrument's results is for students who can utilize their score to create awareness of their own global leadership competencies. Therefore, this research study identified three evidence sources of construct validity: test content, response processes, and internal structure. These three sources of evidence of validity are sufficient to demonstrate inferences for the intended use of the instrument (AERA, 2014). This was conducted in a four-phase process. Phase one established validity evidence based on test content. Phase two established validity evidence based on response processes. Phase three was a pilot study of the instrument. Phase four established validity evidence based on internal structure as well as reliability.

\section{Phase One}

Evidence based on test content "can include logical or empirical analyses of the adequacy with which the test content represents the content domain and of the relevance of the content domain to the proposed interpretation of test scores" (AERA, 2014, p. 14). Essentially, the question is do the items on the instrument sufficiently reflect the concepts it was intended to measure. Subject matter experts evaluate the alignment of items to the subject of the assessment to establish this evidence (AERA, 2014; Messick, 1995). 
Validity evidence based on test content was established by utilizing the specialized knowledge of 13 subject matter experts (AERA, 2014). The subject matter experts were chosen based on their knowledge of the Student Leadership Competencies (2015), global leadership, practical application of leadership programming, a combination of those areas, or a related focus area. The first expert was Dr. Corey Seemiller who is the original researcher and author of the Student Leadership Competencies, an Assistant Professor in the Department of Leadership Studies in Education and Organizations at Wright State University, and was also formerly a director of leadership programs at the University of Arizona. The expert focusing mostly on the global aspects was Dr. Hilary Landorf who is an Associate Professor for International and Intercultural Education, the Director for the FIU Office of Global Learning Initiatives and Executive Director for the Comparative and International Education Society. Two other members of the FIU Global Learning Initiatives office were also included as experts related to the global aspects, Dr. Stephanie Paul Doscher, Associate Director and Eric Feldman, Program Manager. Members of FIU's Center for Leadership and Service (CLS) served as experts as the office uses the Student Leadership Competencies, incorporates global connections because of FIU's Quality Enhancement Plan (Global Learning), and these members are student affairs practitioners. These experts included Dr. Beverly Dalrymple who was the former Executive Director of FIU's CLS and who has researched global leadership, Patricia Lopez- Guerrero, Director, Joanna Garcia, Associate Director, Nashira Williams, Assistant Director, Shannonlee Rodriguez, Assistant Director, Kaleen Martinez, Coordinator, and Peter Melnik, Coordinator. Finally, two other members of my dissertation committee were asked for their perspectives based on their areas of expertise. 
Dr. Thomas Reio is the Assistant Dean of Graduate Studies with research interests in learning motivation-cognitive and sensory curiosity, risk-taking, workplace socialization, workplace incivility, entrepreneurship, and school-to-work transition. Dr. Maria Lovett, Clinical Assistant Professor in the School of Education and Human Development, was asked to take a social justice perspective on the analysis to assist in making the instrument appropriately accessible.

The 13 experts were emailed a letter (see Appendix A) that explained the purpose and process of reviewing the table of specifications, as well as, an excel sheet that contained a table of specifications with two tabs (See Appendices B and C) (I. Newman et al., 2013). Tab one of the table of specifications contained the analysis of the proposed global leadership competencies, which was a breakdown of the global leadership competencies literature (See Appendix B1) and Seemiller's Student Leadership Competencies (See Appendix B2). Tab two contained the hypothesized final ten competencies with their definitions (See Appendix C1) and potential questions to measure those competencies in an unidentified random order (See Appendix C2). The questions were placed in an unidentified random order so that the experts would make their own assumptions about what items matched the competencies instead of following what the researcher was proposing, which strengthens the estimates of content validity (Groves et al., 2011; I. Newman et al., 2013; Thorndike \& Thorndike-Christ, 2010). Dr. Corey Seemiller who had experience in attempting to validate an instrument with questions that were similar to those on tab two, recommended that the questions should be rewritten to focus more on the global leadership literature language than the Student Leadership Competencies language. An email was sent to all experts asking them to only 
give feedback on tab one and to ignore tab two until further notice (See Appendix D). They had approximately a month to complete their analysis.

After receiving and analyzing the feedback of tab one from the 13 experts, new behavioral statements were created for the actual final ten competencies that were determined to be the global leadership framework (AERA, 2014). The new tab two was first sent to Dr. Corey Seemiller to ensure the original concerns were addressed. After some minor revisions based on her feedback, tab two was then sent to the methodologist Dr. Haiying Long, Assistant Professor in Leadership and Professional Studies, who suggested a few more minor revisions. The final version of tab two was then sent out to the 13 subject matter experts (See Appendices E and F). Once their feedback was received, reviewed, and compared, the item pool for the instrument was revised accordingly (I. Newman et al., 2013; Thorndike \& Thorndike-Christ, 2010).

Since there were several wording changes to items based on expert feedback, three experts were requested to review the final results prior to moving into the next phase (AERA, 2014). Dr. Seemiller, Dr. Dalrymple, and Dr. Reio were originally consulted, but due to the time constraint, Dr. Seemiller was unable to assist, and Dr. Doscher was asked instead (See Appendices G and H). The verified statements were then paired with a five-point anchored Likert scale (1 "strongly disagree"; 5 "strongly agree") to estimate the strength of agreement with the behavioral statements for participants (Thorndike \& Thorndike-Christ, 2010).

\section{Phase Two}

Evidence based on response processes comes from individuals in the sample. This is typically established through cognitive interviews, when participants verbalize 
their thoughts while they answer a question in a concurrent think-aloud with probing questions to follow-up on statements that were unclear. Depending on the participant, a retrospective think-aloud was also used, when the participant described how they arrived at their answer. The verbalized thoughts allowed the researcher to help determine if the respondents were interpreting the items on the instrument the way the designer intended by comparing their examples to how they scored themselves, listening for verbal cues of uncertainty or confusion regarding the question, and watching for body language that may also indicate lack of understanding of what is being asked. Cognitive interviews have been widely used to help alleviate misunderstandings in survey questions since 1983, when the concept was introduced at a workshop by the U.S. National Research Council (AERA, 2014; Groves et al., 2011; Messick, 1995). If this method is not used, a pilot may "detect overt problems that disrupt the response elicitation process, but they often do not provide evidence of causes, nor do they provide evidence of covert problems" (Collins, 2003, p. 231).

Cognitive interviews were conducted with 10 FIU students to help determine understanding of the questions. The cognitive interviews helped to determine clarity of the behavioral statements, appropriateness of the statements for the population, whether the language/terminology is understandable, if the questions are appropriate for the scope of the instrument, and whether the information being asked is easily accessible in the students' thought processes (Willis, 2005).

The sample of the students was considered in relation to national demographics. According to the latest report from the National Center for Educational Statistics, in the United States the total fall enrollment in degree-granting postsecondary institutions by 
race/ethnicity of student was 59.3\% White, $15.8 \%$ Hispanic, $14.7 \%$ Black, $6.4 \%$ Asian or Pacific Islander, and 3.8\% are of other origin (Snyder et al., 2016, p. 458). The National Center for Educational Statistics also reported that $57 \%$ of the students were female and 43\% were male (Snyder et al., 2016, p. 407).

To diversify this sample of ten FIU students in age, major, gender, race, and ethnicity so as to reflect the demographics of the population, the executive board members from LEAD Team and Alternative Breaks, which are major FIU CLS programs at both MMC and BBC, as well as office student staff and members of my first-year experience class, were utilized to recruit participants. The researcher asked participants personally to participate and recruited five White, two Hispanic, two Black, and one Asian undergraduate students to be representative of the national breakdown.

Additionally, to represent the national statistics for gender four of the students were men and six were women. There was also an effort to make sure the participants were of varying majors (Communication Arts, Hospitality Management, Criminal Justice and Psychology, Nursing, History, Biology, and Sport Recreation) and ages (18-24).

The cognitive interviews were conducted following the revisions made to the item pool for the instrument based on the feedback from the subject matter experts. The items and scale were inputted into a Qualtrics Survey Software account administered by FIU along with the consent to participate, and examples of activities that help increase the ten global leadership competencies, which appeared after participants received their results (see Appendix I). The ten students were asked to take the instrument on an iPad in front of the researcher and Emani Jerome, a colleague from the Center for Leadership and Service, in an office setting while being verbally recorded. Emani was asked to assist 
with the process to minimize investigator bias, which is where the researcher only sees what they want to see (Willis, 2005). A cognitive interview process guide was created to help Emani as he assisted since the process was new to him (See Appendix J).

Participants were introduced to both researchers, reminded of the purpose of the study, asked if they were comfortable being recorded, assured that both positive and negative comments were helpful, told to ask questions whenever they needed, and then participated in a warm-up activity to help further clarify the think-aloud process. During the warm up activity participants were instructed to "try to visualize the place where you live, and think about how many windows there are in that place. As you count the windows, tell me what you are seeing and thinking about" (Willis, 1994). The researcher then explained that the number of windows was not actually the most useful information in the process. Instead, the way the participant verbally visualized the counting of the windows, such as "now going upstairs, my bedroom is on the right. I see blue walls, no curtains, open window blinds, showing the tree outside" was going to help the most in the cognitive interview process. The participants were told that how they scored themselves on each question was not as important as the reasons they made those scoring decisions.

The researcher began recording after the student completed the demographic information, which is when the participants were asked to read the question out loud then verbalize all the thoughts they were having and any examples they were considering that helped them chose their answer. These thoughts were audio recorded with the participant's permission and the researchers also took notes regarding comments and body language. If either interviewer was unsure about a comment a participant made, follow-up questions or probes were used to help clarify, such as "I haven't heard of that 
program, could you tell us more about it and your participation in it?" or "could you give an example of why you scored yourself that way?" The cognitive interviews lasted between 20 and 40 minutes. The feedback from the cognitive interviews was then used to revise the items on the instrument as needed (Groves et. al., 2011, p. 263-265). The researcher was looking to identify and then address any elements of the questions that could be contributing to response error through this process (Willis, 2005).

\section{Phase Three}

Once both the feedback from subject matter experts and the results of the cognitive interviews were incorporated into the creation of an item pool, a pilot of the instrument was then conducted with a convenience sample of FIU students. The sample was first recruited from two classes taught by the researcher: a first-year experience class and an exploring leadership class. To encourage participation, five extra credit points were given to students that sent a screen shot of their results to the professor within a week. Students were recruited through email for this process (See Appendix K).

The data collected from this pilot were intended to help determine question clarity, questionnaire format, variance in responses and internal validation of items (Babbie, 1990). This sample validation included item-scale correlations, item variance, and reliability. Dimensionality was to be examined and the item pool narrowed based on the all the sample validation results through the use of an exploratory factor analysis (DeVellis, 2016). The first set of results from the pilot test yielded only 28 students. The extra credit assignment was extended by another week and two more classes were asked to participate at this point: another first-year experience class, also offered extra credit, 
and a survey design graduate class, was asked on the basis that there were helping a fellow researcher.

\section{Phase Four}

Evidence based on internal structure is important because it demonstrates how items on the instrument are related to each other and how different parts of an instrument are related. "The conceptual framework for a test may imply a single dimension of behavior, or it may posit several components that are each expected to be homogeneous, but that are also distinct from each other" (AERA, 2014, p. 16). Internal structure evidence can be established through a multivariate statistical method called factor analysis (AERA, 2014; Groves et al., 2011).

Since no major implications, other than having too small of a sample, were discovered during the pilot, the instrument was distributed to a larger sample. Validity evidence based on internal structure utilized the data collected from the larger sample (AERA, 2014). The final sample was recruited by utilizing a snowballing technique (Fraenkel \& Wallen, 2006). An email was sent to 178 professional contacts located all over the USA (see Appendix M) and an announcement was made on my personal Facebook page (see Appendix N). A few days later a graphic was created to help entice people to take the instrument (see Appendix O). The graphic was then posted to social media with a clickable link to the instrument and included in a reminder email sent to the same group of professional contacts a week and a half after the initial email to them (see Appendix P).

The second recruitment email included an emphasis on anyone, not just undergraduate students, taking the instrument. After the first email the researcher had 
many conversations with professional staff who had taken the instrument prior to distributing it to their students, so that they knew what they were sending their students. They found that they learned about themselves in the process then wanted to debrief with me about their results. Consequently, it was decided that collecting data from undergraduate students, graduate students, and others that were college age and above could be beneficial.

To continue to recruit nation-wide for participants, a post was created in the Facebook group for Student Affairs Professionals that has 29,597 members (see Appendix Q). Two comments were made on the post related to the gender options on the instrument indicating that just having the option "other" is seen as non-inclusive. A box beside "other" was added immediately because it could be done mid-data collection since there was no analysis of gender intending to be conducted. Dr. Bronwen Bares Pelaez, Director of FIU's Women's Center and Dr. Gisela P. Vega, Associate Director of Multicultural Programs \& Services, LGBTQA Initiatives were consulted so that the final version of the instrument's gender options could be altered appropriately.

After 719 responses were collected in Qualtrics, an exploratory factor analysis with a principal axis factoring extraction method and a varimax rotation was conducted to discover how many factors/dimensions were present. The analysis was first conducted on all completed responses $(\mathrm{N}=566)$ then the researcher filtered the responses and ran an exploratory factor analysis on different subsets of the data to see how age, being from a different country, and education affected the results. Those subsets included all under 35 $(\mathrm{N}=450)$, all non-international $(\mathrm{N}=543)$, undergraduate and graduate students $(\mathrm{N}=396)$, non-international undergraduate and graduate students $(\mathrm{N}=377)$, undergraduate students 
$(\mathrm{N}=279)$, and non-international undergraduate students $(\mathrm{N}=265)$. All subsets met the recommended sample size minimum of 200 (Meyers, Gamst, \& Guarino, 2013) and KMO and Bartlett's test was also used to ensure sampling adequacy (AERA, 2014; Meyers et al., 2006).

The final sample used in the analysis was undergraduate students $(\mathrm{N}=279)$. Participants were able to choose as many of the race demographics as appropriate, which provided a breakdown of 214 White, 53 Black or African American, 4 American Indian or Alaska Native, 20 Asian, 3 Native Hawaiian or Pacific Islander, and 28 Other. Additionally, 136 also identified as Spanish, Hispanic, or Latinos. The gender breakdown was 78 males, 200 females, and 1 other. Age include 3 under 21, 245 were 18-24, 20 were 25-34, and 11 were over 35. Participants attended 29 different institutions of higher education from across the United States (see Appendix R). There were approximately 145 different majors ranging from Accounting to Women's and Gender Studies.

Cronbach's coefficient alpha was run in SPSS to test for reliability on the same data set (AERA, 2014; Meyers et al., 2006). Table 1 below summarizes the different aspects of validity and reliability, their definition, how evidence for each aspect is provided, and then how that data was used or analyzed in this study. 
Table 1

Research Design

\begin{tabular}{lccc}
\hline \multicolumn{1}{c}{ Aspect } & Definition & $\begin{array}{c}\text { How evidence will be } \\
\text { provided }\end{array}$ & Data Use/Analysis \\
\hline $\begin{array}{l}\text { Validity evidence } \\
\text { based on test } \\
\text { content }\end{array}$ & $\begin{array}{c}\text { Evidence of content } \\
\text { relevance, } \\
\text { representativeness, and } \\
\text { technical quality }\end{array}$ & $\begin{array}{c}\text { Use of subject matter } \\
\text { experts }\end{array}$ & $\begin{array}{c}\text { Compared experts' } \\
\text { feedback on the fit of the } \\
\text { items to the competencies } \\
\text { then revised instrument }\end{array}$ \\
$\begin{array}{l}\text { Validity evidence } \\
\text { based on cognitive } \\
\text { response processes }\end{array}$ & $\begin{array}{c}\text { Theoretical rationales for } \\
\text { the observed consistencies } \\
\text { in test responses }\end{array}$ & $\begin{array}{c}\text { Use of cognitive } \\
\text { interviews }\end{array}$ & $\begin{array}{c}\text { Identified elements of the } \\
\text { items that were } \\
\text { contributing to response } \\
\text { error then revised } \\
\text { instrument }\end{array}$ \\
$\begin{array}{l}\text { Validity evidence } \\
\text { based on internal } \\
\text { structure }\end{array}$ & $\begin{array}{c}\text { The fidelity of the scoring } \\
\text { structure to the structure of } \\
\text { the construct domain at } \\
\text { issue }\end{array}$ & $\begin{array}{c}\text { Results of } \\
\text { exploratory factor } \\
\text { analysis }\end{array}$ & $\begin{array}{c}\text { Determined the number of } \\
\text { factors/dimensions present }\end{array}$ \\
Reliability & The consistency of a & Results of & Cronbach's \\
& measurement procedure & Value of above .7 & \\
\hline
\end{tabular}

\section{Data Analysis Procedures}

The study used an exploratory sequential mixed methods design, where the findings from the qualitative data were used in quantitative data phases. This section is organized by the four phases of research.

\section{Phase One}

Phase one was a qualitative phase used to help establish test content validity evidence, in order to determine if the inferences of the score from the instrument sufficiently reflect the theory it was intended to measure (AERA, 2014). To analyze the feedback from the subject matter experts regarding tab one of the table of specifications, which was my analysis of the global leadership competencies literature and Seemiller's Student Leadership Competencies, all comment columns were placed in one excel 
document (see Appendix R). The experts were asked to comment on both the extent of the fit of the Student Leadership Competency with the global leadership literature in the row and if they thought it was important to address each of the NACE Global/Intercultural Fluency competencies. While each expert made various comments, the researcher determined if the expert agreed with the analysis of the row's data or not. If they did not, the researcher marked the block red. When there was a part of the row that an expert did not agree with although overall seemed to think the competency should be included, the researcher marked those blocks yellow and considered the comments when writing the questions in phase two.

To determine how to categorize the block, the researcher looked for keywords. Experts that agreed included phrases such as "yes," "agree," "relevant," "no further comment," and "fit." Experts that were not in agreement used words such as "no," "missing," "don't," and "not." Blocks that were turned yellow included comments like "partially," "not directly," "some," "seems," and "must also." To help determine which competencies to keep, knowing the researcher wanted ten or less due to survey length considerations (Thorndike \& Thorndike-Christ, 2010), the color-coded blocks were used. The rows that had only one red block were kept since that meant that only one of the experts did not think the competency should be included. The ten competencies that emerged through this process became the global leadership framework mapped within the SLCs.

After determining the ten competencies in the global leadership framework, items were written to measure each competency. There were five items written for each competency (Thorndike \& Thorndike-Christ, 2010). If there was a yellow block, the 
comment made by the expert was considered when writing the item for that competency. For example, if the expert made a comment that the Student Leadership Competencies definition was missing an aspect of the global leadership definitions included in the row, the researcher was sure to include an item that specifically addressed the global leadership aspect that was perceived as missing from the Student Leadership Competencies definition. These 50 items were put into a table of specifications and sent back to the same 13 experts (Newman et al., 2013).

As the responses from the experts came in, they were input into an Excel spreadsheet with the questions color coded to be the same as the competency they were written to evaluate. If an expert indicated that the question could measure a competency, it was tallied. If they had a comment about the question, that was also included in the tally box on the analysis sheet. If at least nine of 12 experts (three-fourths) thought that the item could measure the competency, it was color coded. If it was the competency the item was intended to measure, it was colored purple. If it was not the competency the item was intended to measure, it was colored teal. A $13^{\text {th }}$ expert, who served as the social justice expert, commented only on the wording of the questions instead of matching the questions to competencies. Her remarks are found in the last column on the right and were considered when revising items (see Appendix S).

The Excel spreadsheet was then sorted so all the questions that were intended to measure each competency were grouped together for analysis (see Appendix T). Only three of the five items for each question were desired for the final instrument because of survey length considerations (Thorndike \& Thorndike-Christ, 2010). The decision process for which items were to be included on the pilot instrument were similar for each 
competency. First, at least three-fourths of the experts had to agree that the competency and the item that was written to measure it matched (this was coded purple). Second, the teal blocks were considered. If an item had too many teal blocks, then it was determined that the experts were not certain that the item measured the intended competency since a significant number of them thought it could also measure those other competencies.

Third, the number of experts or tallies in the purple boxes was used to help decide which of the questions were the strongest. Nine experts was the threshold; having all 12 experts agree and match the item and competency was the stronger indicator that the item could measure what it was intended to measure. For three of the competencies four questions were kept for phases two and three because there was a tie during this analysis.

The comments from the experts were then considered and a few wording choices were altered based on the experts' suggestions. This final list of items was then sent to three of the 13 experts for final confirmation of the items being able to measure the intended competency.

\section{Phase Two}

Phase two was another qualitative phase used to help establish validity evidence based on cognitive response processes through cognitive interviews (AERA, 2014). Throughout the cognitive interviews researchers attempted to identify elements of the questions that may contribute to response error (Willis, 2005). While the student was speaking, researchers were looking at both their speech and their body language for indicators such as their understanding of the statements the way it was intended, any words that seemed difficult to understand, words being interpreted differently than 
intended, strong hesitations while trying to recall / answer the question, and very rapid response time - an indication of not giving the question actual consideration.

Positive body language indicators were leaning forward, eye contact with the researchers, and smiling. Negative body language indicators were leaning back, avoiding eye contact, and concern in the face. The hesitations or rapid response time indicators were determined through measuring the time elapsed between the presentation of the question and the indication of a response. These indicators were combined with what the participants were saying to determine if their interpretation was correct or if they were having difficulty with the question. If the participants had positive body language and moderate response time, it allowed the researchers to believe the examples they were giving. The example given then allowed the researcher to compare what the participants thoughts were to what the question was trying to measure. At the end of every cognitive interview, the participants were also asked if they had any comments or concerns about any of the questions or the scale and if they had any other thoughts regarding the survey that they thought might be useful to the researchers (Collins, 2003; Desimone \& Le Floch, 2004).

\section{Phase Three}

The third phase of this research study was to pilot the instrument to help determine questionnaire format, item-scale correlations, item variance, reliability and dimensionality of items (Babbie, 1990; DeVellis, 2016). An examination of the KaiserMeyer Olkin measure was used to determine sampling adequacy, then an exploratory factor analysis with a principal axis factoring extraction method was conducted with the 78 responses to see if there was a need to reduce the number of variables. At first, a 
varimax rotation was unsuccessful, so at this point, three items were removed. When analyzing the results of the experts regarding the items that measured each competency in phase one, Self-Development, Diversity, and Empathy had four questions that were strong, while the other seven competencies had three. Though the researcher was desiring three items per competency to have an appropriate test length (Thorndike \& Thorndike-Christ, 2010), it was decided to keep the four items for those four competencies, then allow the cognitive interviews and possibly the item reduction during the pilot to determine the strongest three items for each competency at that point. When the pilot results did not alter the number of items, the cognitive interview data was then consulted to determine which questions to remove. See Appendix L for the final Qualtrics form.

After removing these questions, another exploratory factor analysis using a principal components extraction method was conducted and a varimax rotation was successfully applied to assist in interpreting the factors (Reio \& Shuck, 2015).

Cronbach's coefficient alpha was also run to test for reliability and used to discover inter item correlations by examining alpha if we were to delete each question. If alpha increased after an item was deleted, the item was not correlated with the other items. Conversely, if alpha decreased, the item was correlated with the other items. (AERA, 2014; Meyers et al., 2006).

\section{Phase Four}

The final phase of this research study was to establish validity evidence based on internal structure as well as evidence of reliability (AERA, 2014). An exploratory factor analysis with a principal axis factoring extraction method with a varimax (orthogonal) 
rotation was conducted in SPSS on data gathered from 279 participants. This process demonstrated how the items relate to each other as well as to the theoretical framework and led to future implications for the instrument since establishing validity is a process (Fabrigar, et al., 1999; Messick, 1989; Williams, et al., 2010).

Factor analysis is a process that assumes that there are measurable variables that can be reduced to fewer latent variables (Yong \& Pearce, 2013). It "can be used to determine what theoretical constructs underlie a given data set and the extent to which these constructs represent the original variables. Of course, the meaningfulness of latent factors is ultimately dependent on researcher definition" (Henson \& Roberts, 2006, p. 396). Exploratory factor analysis (EFA) is "more appropriate during instrument development" (Henson \& Roberts, 2006, p. 409) because "theory often drives item development, and these items are often subsequently assessed with EFA to help refine the assessment” (Henson \& Roberts, 2006, p. 407).

The Kaiser-Meyer-Olkin measure and Bartlett's test of sphericity was first run to determine sampling adequacy. The Kaiser-Meyer-Olkin measure provided an indicator of how adequate the correlations of the sample size are for factor analysis. A value of .70 or higher was needed to help ensure there were enough responses collected to determine if there are factors present. Bartlett's test of sphericity examined the null hypothesis to ensure that none of the variables are significantly correlated. The result of this test needed to be statistically significant (Meyers et al., 2006).

Principal axis factoring (PAF) was chosen as the extraction method because it "explicitly focuses on the common variance among the items, and, therefore, focuses on the latent factor" (Henson \& Roberts, 2006, p. 398). PAF was chosen over a principal 
components analysis because a principal components analysis focuses on explaining the total variance of the variables and only summarizes components into smaller components. These smaller components created in a principal components analysis are calculated without regard to any underlying structure. PAF in contrast is more related to theory development (Costello \& Osborne, 2005; Henson \& Roberts, 2006; Meyers et al., 2006).

Rotations pivot "the first $\mathrm{n}$ number of extracted factors around their point of intersection" (Meyers et al., 2006, p. 667) making the results easier to interpret because it simplifies the data structure. It was decided to use a varimax rotation because it is an orthogonal rotation, which produce factors that are uncorrelated (Costello \& Osborne, 2005). Additionally, varimax rotations are focused on the factors, so it "minimizes the number of variables that have high loadings on each factor and works to make small loadings even smaller" (Yong \& Pearce, 2013, p. 84).

In the SPSS output, the eigenvalues indicated the amount of variance each factor accounted for. In deciding what components to retain, Kaiser's (1960) criterion was used. Kaiser's criterion suggests retaining only those components whose eigenvalues are greater than 1.0. The total variance explained by those values was then examined to see if it was over 50\%. Additionally, the scree test was consulted to examine where the graph was still reasonably dropping and determine if it showed the same number of factors identified in the table. Factor loadings were analyzed. Usually the item with the largest factor loading is what is put into that the item. While the factor loading numbers are used for the decision of keeping or deleting the item, the theory must also be considered in final decisions. New labels were created as needed (Costello \& Osborne, 2005; Meyers et al., 2006; Yong \& Pearce, 2013). 
Cronbach's coefficient alpha was run to test for reliability. It was examined for the instrument overall and each of the factors discovered. A value of 7 or above was strived for as according to Meyers et al., (2006)

.90 or better is outstanding, high to middle $.8 \mathrm{~s}$ is very good, .80 or the low $.8 \mathrm{~s}$ is good, high to middle $.7 \mathrm{~s}$ is acceptable, .70 or the low $.7 \mathrm{~s}$ is borderline acceptable, high to middle .6s may be ok for research purposes, the low .6s are problematic, and anything below that is not acceptable (p. 722).

\section{Summary}

This chapter reviewed the methods that were used in this study. It included the research questions, the relevant aspects of validity and reliability, and the research design, descriptions of the samples, data collection procedures, and data analysis procedures. The study used an exploratory sequential mixed methods design. An instrument was developed from four phases - two qualitative and two quantitative. Chapter 4 reviews the results of the research using these methods. 


\section{CHAPTER IV}

\section{RESULTS}

The purpose of this study was to develop a self-assessment instrument with psychometrically sound reliability and validity inferences to measure global leadership competencies. Data were collected and analyzed to answer the study's two research questions:

1. Does the Global Leadership Competencies self-assessment instrument yield valid inferences about students' global leadership competencies?

2. Does the Global Leadership Competencies self-assessment instrument yield reliable inferences about students' global leadership competencies?

The study used an exploratory sequential mixed methods design, where the findings from the qualitative data were used for the subsequent quantitative data phases. The results are organized by the four phases of research conducted. Chapter 4 presents a description and analysis of the data collected to test each of the research questions. It includes analysis of the results for each of the four phases of the study and a summary of findings.

\section{Phase One}

Phase one was a qualitative phase used to help establish test content validity evidence, which determined if the inferences of the score from the instrument sufficiently reflect the theory it was intended to measure (AERA, 2014). Tab one of the spreadsheet had a table of specifications on which was recorded in the column boxes the opinions and thoughts the 13 experts on how each of the Student Leadership Competency definition in the column on the left two fit with the global leadership concepts in that same row. Their 
comments were analyzed then their comment box was color coded to red if they did not agree, yellow if they partially agreed, and white if they agreed (see Appendix S).

The first component/construct, Responding to Change, had one red, two yellow, and ten white boxes after the analysis. The expert that was coded as red commented "I don't think this competency relates to nonjudgmentalness as indicated in some of the definitions. It concerns situations much more than people." The two experts that were maybes wrote, "The items in red appear to be more about suspending judgment or having open perspectives rather than responding to change" and "This definition does not get at the nonjudgmental part; one needs to be both flexible and nonjudgmental when responding to change. This would be important to include." The overall comments of those that agreed were strong such as "Yes -The SLC mentions abilities (being flexible and positive) and actions (adapting quickly, creating smooth transitions and moving forward). Similar abilities and actions are mentioned in the other definitions," "I agree that this definition subsumes the literature in this row," and "I agree that the definition of responding to change adequately reflects the information contained in this row." With overall agreement, it was decided to include this competency in the framework.

Self-Development, was also coded to have one red, two yellow, and ten white boxes. The red box comment reads, "These descriptors fit more with the SLC, Other Perspectives, rather than Self-Development." The two comments coded yellow were "This definition does not get at the curiosity and openness to new ideas that selfdevelopment entails. Self-development must also consist of self-directed learning and it is lifelong. The development part is missing from the current definition." And "For GCI, GMI, ISS, ICAPS and MPQ all speak about openness. I'm not sure how well openness 
fits into the definition of self-development. I think openness is a component of selfdevelopment I wonder if openness would fit better under diversity." Comments coded as white read "Consistent through all of the explanations"" "Yes I like this point that leaders are always learning too. It is an intentional, strategic aspect of their practice," and "Yes the desire to learn is evident in the other definitions." This competency was kept for the framework, but when writing the items the comments about "life-long learning" were consulted and used.

Responding to Ambiguity had one yellow and 12 white boxes after analysis. The yellow comment was "confused about this one with regards to risk. Growth comes from the unknown experiences but enjoying the challenge depends on the individual. Box D I don't agree with." The rest of the comments were along the lines of "Consistent through all of the explanations," "Besides respond I believe there is some value to include the wording of adapt," and "Yes, I think embracing and welcoming the uncertainty is an important part of leadership. The quest for 'adventure' comments make me pause however, sounds self-indulgent and I question the motive then.” This competency was kept for the framework and the word "adventure" was avoided when writing the items.

Systems Thinking had four red, two yellow, and seven white boxes. The red comments were stronger than the white comments for this competency. Some red comments were "I think this definition is associated with the literature here, but I don't think it subsumes it in the way that the literature is subsumed by the SLC definitions above. Although 'systems thinking' is not necessarily associated with globalization or global dynamics in the definition, I think you have to be a systems thinking to grasp the meaning and effects of globalization and, if you "get" globalization, you are probably a 
systems thinker in terms of organizational leadership. This is the case even if the leader is only thinking about systems within one complex organization dealing with only local or domestic issues." And "Columns M and P are most relevant because they explicitly reference systems, structures, and organization. Others refer to diverse ways of thinking which may be a predecessor to systems thinking but don't address the systems and how to lead within them (most of these are probably better for the other perspectives' definition below)." Whereas the white comments were vague such as "This is reflective of some roles I believe. As a department head you may be thinking Globally or big picture. In many positions, the role may limited itself one scoop is limited because of access and what you will or can be exposure too." White boxes comments included "No additional comments" or no comments at all. This competency was not included in the framework.

Others' Perspectives had one red, two yellow, and ten white boxes. The box coded red stated, "I do not get a sense of a global attitude here. Leaders need to both seek and embrace other perspectives. This would be important to include when thinking about the NACE definition." The boxes coded yellow included "Partially - All of the definitions recognize the "other" but the SLC also includes for what end ("to develop better solutions/approaches"). This aspect does not appear to be represented in the other definitions." And "The CCAI explanation seems to be too basic for what the SLO is asking from a leader. The valuation is not the same as 'truly considering other options' and isn't as strong as the diplomacy and, cosmopolitanism and other examples used." Some boxes coded white stated "Yes well said and very important point. You don't know what you don't know...so you have to seek experiences and people to teach you." And 
"incorporate respect or respectfulness and appreciation of differences". With most of the experts agreeing that this competency did fit, this competency was kept, though culture was used as a focus when writing items for this to address the experts' comments here and in other places.

Diversity also had one red, two yellow and ten white boxes. The red box declared "The intercultural part seems to be missing, as well as the curiosity and passion for diversity. We cannot move forward in any significant way if we do not honor diversity. Period." The yellow boxes read "I do not think that the literature from the "integrated global leadership competency model' falls under this definition. I think that one can be inquisitive about some things but not be so about other things, such as others' backgrounds, beliefs, or experiences." And "Column P is too focused on geography people from the same place as you can be diverse. The green definition itself seems flawed because it says people will find themselves working with different people but does not say how they will be effective at it." The white boxes included comments such as 'Yes - SLC describes ability to work with others who are 'different.' This is represented in the other definitions." This competency was included, but culture was used instead of the word diversity to address some of the intercultural concerns when writing the items and the word curiosity was also included in an item.

Productive relationships had two yellow coded boxes and 11 white boxes. The comments in the yellow boxes were "I think that some of the literature in this section address the SLC, but some are developmental to it. The SLC specifically says that simply interacting with people does not constitute a relationship; some of the literature in the section describe effective interaction, but only interaction, nonetheless. I think the 
literature that closely aligns with the outcome are in columns C, M, and N." And "Highlight ability and willingness to listen and be respectful. What does meaningful mean? Extraversion really does not fit because it is not a competency. Cross-cultural part is missing." Examples of comments in the white boxes were "Yes - SLC describes this as the ability to make meaningful connections. Collectively, the other definitions support effective interactions." And "Consistent through all of the explanations." This competency was kept for the framework.

All experts agreed on empathy. Comments read "Yes - the combined definitions address the ability to understand the feelings/emotions of others." As well as, "this literature is all closely aligned with the SLC." This competency was included in the framework.

Self-Understanding only had one yellow box that stated "Columns C and O apply. The others are not reflected by the definition of self-understanding provided here." The rest were coded white and the comments were "Yes - self-awareness is represented in the other definitions." And "Yes well stated. Mindfulness may also be a word to include and intentional self-reflection." This competency was also included.

Others' Circumstances had four red boxes, two yellow boxes, and seven white boxes. The red boxes had strong comments like "No - the understanding of the situations/conditions that affect others is not clearly described by the other definitions. This SLC appears to be different from Others' Perspectives." "I do not think that any of the literature addresses this SLC. According to my understanding, this SLC does not deal with culture per se, but rather the conditions within which one is living. This involves such things as family issues, living conditions, economic struggles, and other contextual 
factors that influence people's ability to work and interact with others. This goes beyond culture to a more personal understanding of others' lived circumstances." The white boxes contained no comments or simply indicated that the experts agreed. This competency was not kept.

Positive attitude had one red, two yellow, and ten white boxes. The comment in the red box stated "This does not get at optimism. Fostering a sense of optimism is not the same as being optimistic." The yellow coded comments declared "Partially - Being hopeful or optimistic is mentioned in the other definitions. However, the SLC also includes the ability to inspire or have an impact on others. This aspect of the SLC is not represented." And "The idea of having a positive outlook does not mean that it may lead to positive results. Optimism is the idea that good will prevail. The can-do attitude emerges during complicated situations will help with the current situations but Openness should be considered as well." It should be noted that at this point in the table of specification and onwards, if the experts agreed they would to just say that they agree or left the boxes blank instead of explaining why. This competency was kept.

Confidence had three red, two yellow, and eight white boxes. Some of the red box coded comments were "I'm not crazy about this one. Per my comment above regarding Moses and Baker (who I am sure people would describe as confident) but this definition separates the individual from the group and I definitely don't like the use of the word followers. Re-work this. Or take it out. I think similar competencies are reflected in other areas." And "The only thing that stood out was the usage of he in one for the CCAI. This SLO is aligned more with the first sentence of global/intercultural fluency. I think the Western understanding of confidence does not necessarily comply with 
'demonstrates, openness, inclusiveness, sensitivity, and the ability to interact respectfully with all people and understand individuals' differences', but I think including the CCAI 'ability to respect differing cultural values' is helpful to the definition or description of the SLO." And the yellow coded box comment was "Competence is the precursor to confidence. Thus, intercultural confidence must follow being interculturally competent. Competence is a must in this definition." Without any strong reasons to keep this competency in any of the white boxes, it was decided not to include this competency in the framework.

Resiliency had one yellow and 12 white coded boxes. The yellow box comment read "All definitions are relevant. A difference though is that the green definition seems to focus on failures (something you tried and didn't succeed at) and the others focus on difficulties more broadly including ones that were not a result of one's leadership endeavors." There was some positive reasoning included in the white box comments for this competency such as "Resiliency is being able to roll with the punches and move positively forward, especially when confronted with those issues arising when intercultural conflicts occur." And "Yes excellent. I think of perseverance, and of tenacity too." This competency was included in the framework.

There were 12 other competencies in the table, but most of those twelve only had one of the global leadership theories in the row, so experts did not analyze them. Those competencies included personal values, organization, problem solving, group development, verbal, vision, motivation, scope of competence, research, organizational behavior, ethics, and power dynamics. 
From this analysis, the researcher concluded that the following competencies comprise the global leadership framework for the Student Leadership Competencies:

(a) Responding to Change, (b) Self-Development, (c) Responding to Ambiguity,

(d) Others Perspectives, (e) Diversity, (f) Productive Relationships, (g) Empathy,

(h) Self-Understanding, (i) Positive Attitude, and (j) Resiliency.

After the framework was decided, five items for each competency were written and placed into another table of specifications. The results from the experts were tallied; if a comment was made it was included, and then color coded. Purple indicated that three-fourths of the experts agreed and it was the item written for that competency. Teal indicated that three-fourths of the experts agreed, but the item was not written for that competency (See Appendix U).

All five of the Responding to Change items were coded purple, but three of them were coded teal for Responding to Ambiguity and one was coded teal in Empathy. Though all five were coded purple, the three with the highest tallies were kept. All five of the items written for Self-Development were also purple and no other blocks were teal. Four of the questions had 11 or 12 in the tallies, so all four were kept. Four of the five Responding to Ambiguity items were purple with one item coded teal in Resiliency. The three questions that were purple and did not cross load into Resiliency were kept. One of the experts commented "what is appropriately," so it was decided to change that phrase with "remain open to new information."

Three of the Others' Perspectives questions were coded purple, but four of them were coded teal for Diversity. "Culture" was originally used instead of "Others' Perspectives" to attempt to address the comments made by experts regarding that piece 
being missing, but the phrases were swapped out when the word culture had the questions leaning too much toward Diversity. The three purple questions, with adjusted wording, were kept. Four of the Diversity questions were coded purple, but those same questions were also coded teal for others' perspectives. Since the substitution was made for the items in Others' Perspectives, it was decided to keep all four with no wording changes.

Productive Relationships also had all five items coded purple, though one item loaded teal for both Others' Perspectives and Diversity. Another was also coded teal for Others' Perspectives. The three questions that did not have a teal block were kept. Four of the items for Empathy were coded purple. The one not coded purple was teal for Others' Perspectives and Diversity. One question also had a teal block for Other's Perspectives and a different question was teal for Diversity. All four purple Empathy items were kept because they had such high tallies. All five of the Self-Understanding items were coded purple with one having a teal block. The three with the highest tallies were kept, which did not include the question that had the teal block for Diversity. All five of the Positive Attitude items were purple with not teal blocks. The four questions that all experts agreed on were kept. Four of the Resiliency questions were coded purple. Two of the questions had teal blocks for both Responding to Change and Ambiguity and, these were removed. There were two other questions that had teal blocks for Responding to Change and the other question had a teal block for Positive Attitude.

This slightly modified final list of items was then sent to three of the experts for final review. All three experts agreed that the items were appropriate. That final list of items can be found in Appendix H. 


\section{Phase Two}

Phase two was also a qualitative phase used to establish validity evidence based on cognitive response processes through cognitive interviews. This allowed the researcher to help determine if the respondents are interpreting the items and evaluating themselves appropriately the way the designer intended (AERA, 2014; Groves et al., 2011; Messick, 1995).

Response time can be one indicator of question cognition. In this study, participants tended to have similar response times for all questions. If they took their time / hesitated to answer, they did that for most questions. If they answered quickly, they also did that for most questions. The ten participants varied on their response times.

Researchers determined that the participants could understand and appropriately respond to all the statements. They came to this conclusion by comparing the examples given by participants to the competency being measured to decide if the participants understood the statement and assessed themselves reasonably. An example that reflected understanding of the statements they answered as well as an appropriate self-evaluation is when answering the question "I am aware of my emotions during interactions with other people" one participant ranked themselves as "slightly agree" because "my face does not always go with what I am thinking, so I send mix messages." This was one of the items intended to measure Empathy. In items related to Empathy several participants also commented about emotional intelligence as they were ranking themselves. They were either very comfortable or very uncomfortable with this concept as they discussed how it related to how they thought they scored. This comfort or discomfort was further confirmed through their body language. 
Items measuring Productive Relationships allowed participants to talk about situations from work, school, family, and intimate partner relationships. One participant answered, "I am building a network of people that can support and guide me" as "strongly agree" because they are in their "fourth year at FIU and have been purposeful on what involvements and leadership positions I've taken each year. Each experience has given me a different group of individuals that support and guide me in different ways."

When participants were responding to questions that related to the Responding to Change competency, they talked about how they felt - either being uneasy or relaxed, discussed the amount of effort needed depending on the situation, and described tactics they use when faced with change. Their comfort or discomfort with this set of questions was also reflected in their body language - either leaning in and excited or backing away from the table with the iPad on it as they spoke about it. This again helped the researcher analyze if the example and self-score matched the action.

The items related to the Self-Development competency produced responses such as "learning is the only thing that will allow you to excel." For these items most students responded instantly and we had to probe to have them explain or give an example of why they gave themselves that score, such as "give us an example of a new experience you exposed yourself to." At that point the participants affirmed that their participation in college or leadership co-curricular activities was related to improving themselves, such as "I joined SGA because it was a different type of involvement compared to my other community service focused organizations, so I knew I would learn from it."

The Others' Perspectives" items had participants talking about how they only know the world as they see it so it is important to solicit views or opinions from others to 
make a result stronger. Probes such as, "how did you use that perspective once you got it?" were used to help the researchers understand if they were not only aware of needing the perspective but also able to use it appropriately as well. Some of the participants discussed these questions with a social justice lens. For example, one participant responded, "it is so important to not take peoples' voices away."

Participants reactions to the Diversity items included examples of interactions they had with people they identified as different from themselves and appreciating learning from them. Two of the participants who came from the middle-west claimed that the diversity present at FIU was one of the main reasons they chose to attend. Probes were used such as "how has being around diversity impacted you?" on the basis that simply being surrounded by diversity is not the same as engaging in or learning from it. One participant said, "in group projects I notice that we each bring a different perspective because of our diversity, while it makes working together challenging, it also makes our project better."

Notably, the Self-Understanding items solicited negative examples of the ways the participants had gone through a situation, but all came out of that situation better for going through it. The phrase "out of my comfort zone" was also used by several of the participants in describing their examples. The body language of the participants as they answered was almost opposite of the discomfort indicators some of the other questions solicited. They were giving negative examples, but demonstrating positive body language by leaning forward and making eye contact with the researchers. This led us to believe they had genuinely learned from those situations, making them confident when telling their story. For example, a participant stated, "I understand my strengths and 
weaknesses, but I find that I learn more later and sometimes they conflict. SGA is a more formal leadership position and while I consider my flowery, friendly nature a strength normally, it is a weakness when people use it against me."

The Positive Attitude items allowed participants to describe how they make situations better through both actions and changing their thinking. Responding to the item about having a "can do attitude" one participant said, "I am motivated to the best I can even when it is hard." Several of the responses from participants were "I try" or "I want to" or "I know I should" and then gave an excuse as to why they do not stay positive consistently. While they may have been hard on themselves in their explanations, they did score themselves appropriately as slightly agree or agree.

After all the cognitive interviews were conducted, the researchers decided two words should be altered because participants needed clarification on them or that they were interpreted differently than intended. Five of the ten participants asked for clarification on what "ambiguity" meant, although they understood the phrase "ambiguous situation" to mean an uncertain situation. Four of the ten participants struggled with the word "resilient" either needing clarification or interpreting it to mean something it did not. A few of the participants that did not verbally express their uneasiness with either or both of those words indicated discomfort with their body language. They made puzzled faces or sudden body movements before responding. While the participants understood the overall statement after the researchers clarified those words, it became clear that those two words needed be replaced.

Therefore, "I react comfortably to ambiguity" was changed to "I react comfortably to uncertainty." "I am resilient when things don't go the way I hoped" was 
changed to "I am able to rise again when things don't go the way I hoped." The researcher asked two additional students to do a think aloud process for just those two questions. These students were recruited because they were in the Center for Leadership and Service office waiting for a program to begin. They demonstrated understanding of the questions. See Appendix V for the full question list after the cognitive interviews.

The cognitive interviews in this phase also helped narrow down the item pool during the pilot study in phase three. From Self-Development the item "I enjoy learning about new things" was removed because several participants reacted "of course" instead of giving an example that demonstrated the competency like the other items. From Diversity the item "I interact with individuals who have different backgrounds, beliefs, and/or experiences than I do" was removed because a few participants indicated that they were forced into these interactions through work or class, which is not as good a measure of their competency level as other items where they voluntarily interacted. From Empathy the item "I consider other people's feelings when making decisions" was removed because some participants reused the same example they used when answering a previous item in two different competency groups. Finally, from Positive Attitude the item "I maintain a positive outlook" was removed because this is the question that participants were the hardest on themselves about not doing it all the time, though they otherwise demonstrated it in a competent way.

\section{Phase Three}

The third phase of this research study was to pilot the instrument so as to determine questionnaire format, item-scale correlations, item variance, reliability and dimensionality of items (Babbie, 1990; DeVellis, 2016). The item that got the highest 
score was "I understand my personal values and beliefs" with a mean of 5.53 and standard deviation of .675. The item "I react comfortably to uncertainty" had the lowest score and also had the largest standard deviation with a mean of 3.80 and standard deviation of 1.246. Table 3 shows the rest of the descriptive statistics for the pilot study. An examination of the Kaiser-Meyer Olkin measure of sampling adequacy demonstrated that the sample was factorable $(\mathrm{KMO}=.795)$ since Kaiser $(1970)$ recommends a value of .70 or above, but it did not meet a minimum suggested sample of 200 (Meyers et al., 2006). When an exploratory factor analysis with a principal axis factoring extraction was conducted on the data, eight factors emerged, but when a varimax rotation was applied the rotation failed. At this point, four questions were removed based on the cognitive interview data and after reviewing the inter item correlations. The items removed were "I enjoy learning about new things," "I interact with individuals who have different backgrounds, beliefs, and/or experiences than I do," "I consider other people's feelings when making decisions," and "I maintain a positive outlook." Removing the recommended four questions based on the cognitive interviews neither helped nor hurt Cronbach's Alpha, which is shown in Table 2. Cronbach's Alpha for the entire instrument was .931. See Appendix W for the final list of items included on the instrument. 
Table 2

Inter Item Correlations for Pilot Study

Cronbach's

Item

Alpha if Item

Deleted

I adjust my behavior when something occurs that is out of my control.

.932

I am committed to life-long learning.

.932

I react comfortably to uncertainty.

I consider others' perspectives when making decisions.

.931

I am curious about different cultures.

.934

I am willing to take time to develop productive relationships.

.932

I emotionally connect with people who have experiences different from my own.

I am aware of my emotions during interactions with other people.

.935

I demonstrate a can-do attitude.

I quickly bounce back from failures.

I implement a new plan when a change occurs.

I enjoy learning about new things.

.931

I remain open to new information in uncertain situations.

.931

I show interest in others' perspectives.

.932

I appreciate the differences of other cultures.

.931

I am building a network of people that can support and guide me.

.932

I put myself in others' situations.

I understand my personal values and beliefs.

I maintain a positive outlook. 
Item

Cronbach's

Alpha if Item

Deleted

I look for new opportunities to improve myself.

I respond calmly in ambiguous situations.

I am willing to adapt my perspective to incorporate others' perspectives when necessary.

I enjoy exploring cultures other than my own.

I maintain productive relationships.

I understand my strengths and weaknesses.

I remain positive in challenging situations.

I am able to rise again when things don't go the way I hoped.

I expose myself to new experiences.

I interact with individuals who have different backgrounds, beliefs, and/or experiences than I do.

I consider other people's feelings when making decisions.

I role model a positive attitude for others in difficult situations.

.930

Note: The bolded items were removed from the final instrument.

After removing the four items, another exploratory factor analysis with a principal axis factoring extraction was run with the pilot data again. This time seven factors emerged and a varimax rotation could be applied. The rotated eigen values showed that the first factor explained $12.505 \%$ of the variance of the factor, the second factor $10.506 \%$ of the variance, the third factor $10.377 \%$ of the variance, the fourth factor $8.861 \%$ of the variance, the fifth factor $7.203 \%$ of the variance, the sixth factor $6.857 \%$ of the variance, and the seventh factor $3.584 \%$ of the variance. These seven factors accounted for $59.894 \%$ of the total variance. Additionally, the scree test was consulted and it yielded a similar seven components (Figure 1). 


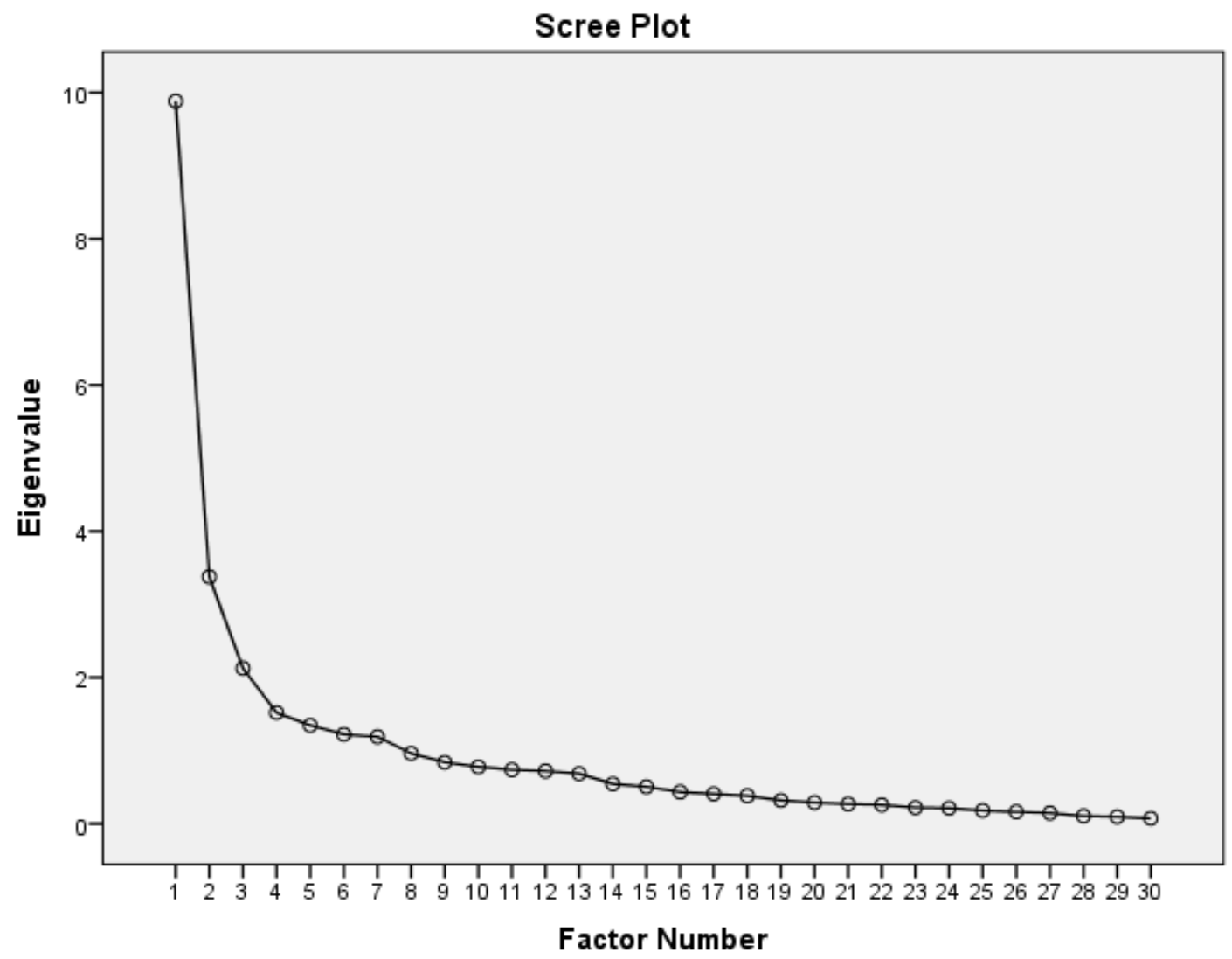

Figure 1. Pilot Study Scree Plot. This figure illustrates the pilot data's scree plot output from SPSS.

Table 3

Descriptive Statistics for Pilot Study $(N=70)$

\begin{tabular}{lcc}
\hline \multicolumn{1}{c}{ Item } & Mean & $\begin{array}{l}\text { Std. } \\
\text { dev. }\end{array}$ \\
\hline $\begin{array}{l}\text { I adjust my behavior when something occurs that is out of my } \\
\text { control. }\end{array}$ & 4.93 & .644 \\
I am committed to life-long learning. & 5.47 & .696 \\
I react comfortably to uncertainty. & 3.80 & 1.246 \\
I consider others' perspectives when making decisions. & 5.16 & .810
\end{tabular}


I am willing to take time to develop productive relationships.

I emotionally connect with people who have experiences different from my own.

$\begin{array}{llll}\text { I am aware of my emotions during interactions with other people. } & 5.04 \quad 806\end{array}$

I demonstrate a can-do attitude.

I quickly bounce back from failures.

I implement a new plan when a change occurs.

I remain open to new information in uncertain situations.

I show interest in others' perspectives.

I appreciate the differences of other cultures.

I am building a network of people that can support and guide me.

I understand my personal values and beliefs.

I recover from setbacks.

I can be flexible when a change occurs.

I look for new opportunities to improve myself.

I respond calmly in ambiguous situations. 
(Table 3 continued)

Item

Mean

Std.

dev.

I enjoy exploring cultures other than my own.

$5.27 \quad .931$

I maintain productive relationships.

$5.20 \quad .734$

I am empathetic toward others.

$5.40 \quad .668$

I understand my strengths and weaknesses.

5.17

.659

I remain positive in challenging situations.

$4.91 \quad 1.004$

I am able to rise again when things don't go the way I hoped.

5.01

.825

I expose myself to new experiences.

I role model a positive attitude for others in difficult situations.

4.97

.851

The factor loadings presented in Table 4 shows that some items clearly loaded on some factors and several of the items cross-loaded into multiple factors. Based only on the primary loading, which is bolded in Table 4, Positive Attitude, Responding to Change, Others' Perspectives, and Self-Development items load into factor one. Factor two included items from Diversity, Empathy, Self-Understanding, and Productive Relationships. Factor three had items from Resiliency, Responding to Ambiguity, and Responding to Change. Factor four loaded items from Responding to Ambiguity, Diversity, and Self-Development. Factor five included items from Self-Understanding and Productive Relationships. Factor six had items from Others' Perspectives, and SelfUnderstanding. Finally, the seventh factor loaded an item from Self-Understanding. Reliability on the overall instrument was excellent with Cronbach's coefficient alpha 
being .934 (Meyers et al., 2006). Since the sample size was small, but some of the theoretical factors were still emerging and reliability was good, it was decided to move on to the final phase of research (Fabrigar, et al., 1999; Henson \& Roberts, 2006;

Williams, et al., 2010; Yong \& Pearce, 2013).

Table 4

Summary of Exploratory Factor Analysis Results for Pilot Study $(N=70)$

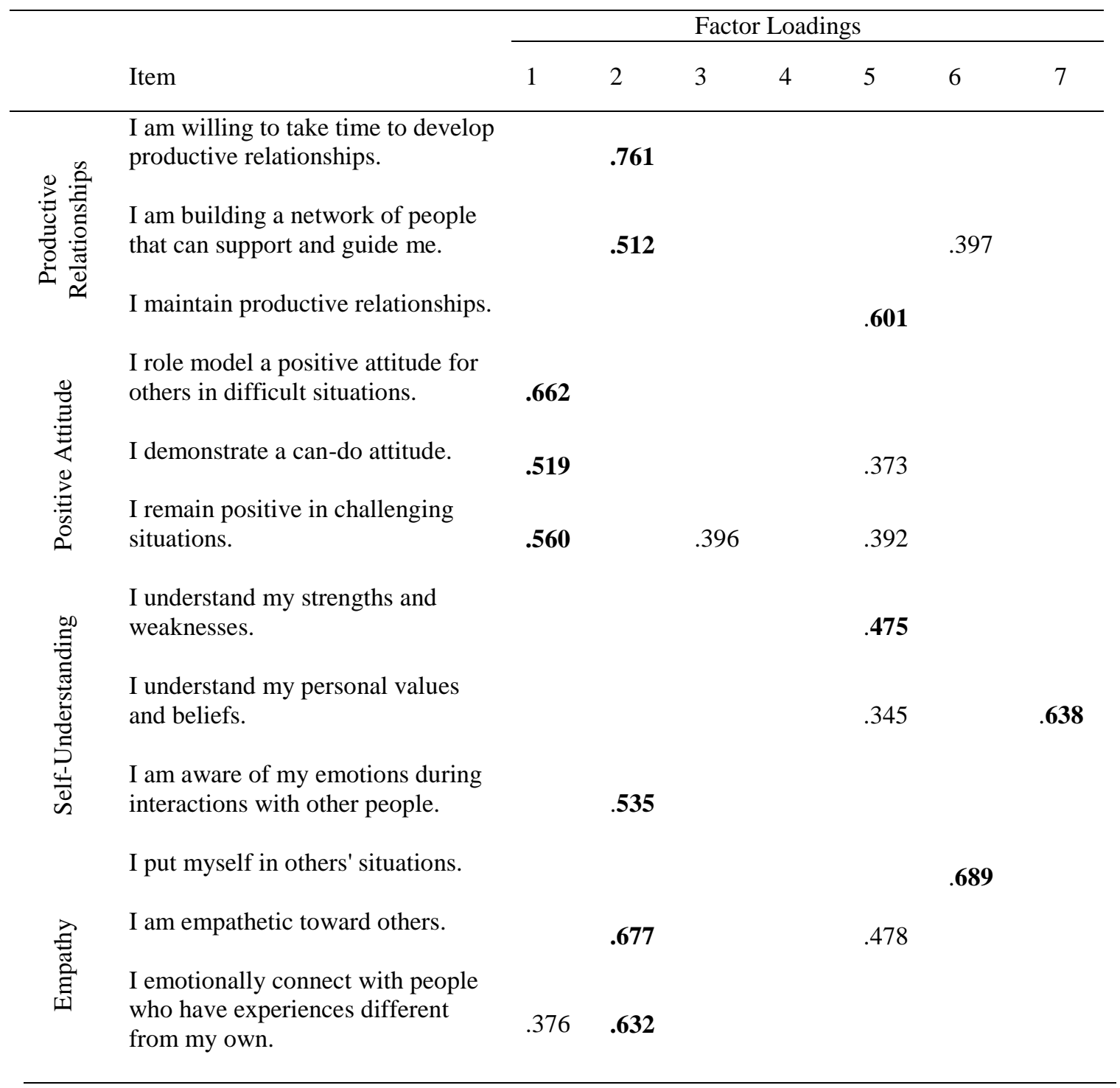

(Table 4 Continues) 


\begin{tabular}{|c|c|c|c|c|c|c|c|c|}
\hline & & & & & or Loa & ings & & \\
\hline & Item & 1 & 2 & 3 & 4 & 5 & 6 & 7 \\
\hline 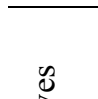 & $\begin{array}{l}\text { I consider others' perspectives } \\
\text { when making decisions. }\end{array}$ & & .398 & & & & .409 & \\
\hline 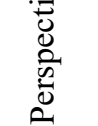 & $\begin{array}{l}\text { I show interest in others' } \\
\text { perspectives. }\end{array}$ & & & & .351 & & .657 & \\
\hline in & $\begin{array}{l}\text { I am willing to adapt my } \\
\text { perspective to incorporate others' } \\
\text { perspectives when necessary. }\end{array}$ & .492 & & & & & & \\
\hline 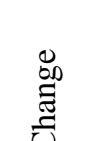 & $\begin{array}{l}\text { I adjust my behavior when } \\
\text { something occurs that is out of my } \\
\text { control. }\end{array}$ & .757 & & & & & & \\
\hline$\stackrel{\ominus}{\stackrel{0}{0}}$ & $\begin{array}{l}\text { I implement a new plan when a } \\
\text { change occurs. }\end{array}$ & .537 & & & .330 & & & .424 \\
\hline 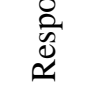 & $\begin{array}{l}\text { I can be flexible when a change } \\
\text { occurs. }\end{array}$ & .318 & & .421 & & .396 & & \\
\hline$\vec{\Xi}$ & $\begin{array}{l}\text { I am committed to life-long } \\
\text { learning. }\end{array}$ & .493 & & & & .350 & .306 & \\
\hline $\begin{array}{l}\frac{0}{0} \\
\stackrel{0}{ \pm}\end{array}$ & $\begin{array}{l}\text { I look for new opportunities to } \\
\text { improve myself. }\end{array}$ & .514 & & & .468 & & & \\
\hline$\overline{\bar{D}}$ & I expose myself to new experiences. & .383 & & .320 & .403 & & & \\
\hline & $\begin{array}{l}\text { I am curious about different } \\
\text { cultures. }\end{array}$ & & & & .806 & & & \\
\hline 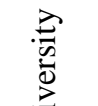 & $\begin{array}{l}\text { I appreciate the differences of other } \\
\text { cultures. }\end{array}$ & & .559 & & .482 & & & \\
\hline & $\begin{array}{l}\text { I enjoy exploring cultures other } \\
\text { than my own. }\end{array}$ & & .397 & & .674 & & & \\
\hline & I react comfortably to uncertainty. & & & .418 & & & & -.332 \\
\hline 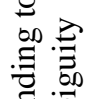 & $\begin{array}{l}\text { I remain open to new information } \\
\text { in uncertain situations. }\end{array}$ & .387 & & & .443 & & .316 & .369 \\
\hline $\begin{array}{l}0^{2} \\
2 \\
2\end{array}$ & $\begin{array}{l}\text { I respond calmly in ambiguous } \\
\text { situations. }\end{array}$ & & & .509 & & .322 & & \\
\hline
\end{tabular}




\begin{tabular}{|c|c|c|c|c|c|c|c|c|}
\hline & \multirow[b]{2}{*}{ Item } & \multicolumn{7}{|c|}{ Factor Loadings } \\
\hline & & 1 & 2 & 3 & 4 & 5 & 6 & 7 \\
\hline \multirow{6}{*}{ 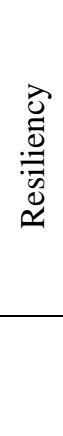 } & $\begin{array}{l}\text { I quickly bounce back from } \\
\text { failures. }\end{array}$ & & & .877 & & & & \\
\hline & I recover from setbacks. & & & .778 & & & & \\
\hline & $\begin{array}{l}\text { I am able to rise again when things } \\
\text { don't go the way I hoped. }\end{array}$ & & & .616 & & & & \\
\hline & Eigenvalues & 9.88 & 3.38 & 2.13 & 1.52 & 1.35 & 1.22 & 1.19 \\
\hline & $\%$ of variance & 12.51 & 10.51 & 10.38 & 8.86 & 7.20 & 6.86 & 3.58 \\
\hline & Cumulative \% & 12.51 & 23.01 & 33.39 & 42.25 & 49.45 & 56.31 & 59.89 \\
\hline
\end{tabular}

Note: Factor loadings $<.30$ are suppressed.

\section{Phase Four}

The final phase of this research study was to establish validity evidence based on internal structure as well as evidence of reliability (AERA, 2014). An exploratory factor analysis principal axis factor with a varimax rotation was conducted in SPSS on data gathered from 279 participants. Reliability was also examined in this phase using Cronbach's Alpha on the overall instrument as well as each construct that emerged. The item with the highest score in this phase was "I am committed to life-long learning" with a mean of 5.55 and a standard deviation of .780. "I react comfortably to uncertainty" was also the lowest score and highest standard deviation with a mean of 3.78 and standard deviation of 1.365 as seen in Table 5. 
Table 5

Descriptive Statistics for O'Keefe Global Leadership Assessment $(N=279)$

\begin{tabular}{|c|c|c|}
\hline Item & Mean & $\begin{array}{l}\text { Std. } \\
\text { dev. }\end{array}$ \\
\hline $\begin{array}{l}\text { I adjust my behavior when something occurs that is out of my } \\
\text { control. }\end{array}$ & 4.63 & 1.019 \\
\hline I am committed to life-long learning. & 5.55 & .780 \\
\hline I react comfortably to uncertainty. & 3.78 & 1.365 \\
\hline I consider others' perspectives when making decisions. & 5.22 & .798 \\
\hline I am curious about different cultures. & 5.48 & .877 \\
\hline I am willing to take time to develop productive relationships. & 5.43 & .769 \\
\hline $\begin{array}{l}\text { I emotionally connect with people who have experiences } \\
\text { different from my own. }\end{array}$ & 4.95 & .984 \\
\hline $\begin{array}{l}\text { I am aware of my emotions during interactions with other } \\
\text { people. }\end{array}$ & 5.04 & .936 \\
\hline I demonstrate a can-do attitude. & 5.23 & .938 \\
\hline I quickly bounce back from failures. & 4.64 & 1.056 \\
\hline I implement a new plan when a change occurs. & 5.02 & .804 \\
\hline I remain open to new information in uncertain situations. & 5.18 & .793 \\
\hline I show interest in others' perspectives. & 5.32 & .707 \\
\hline I appreciate the differences of other cultures. & 5.44 & .815 \\
\hline $\begin{array}{l}\text { I am building a network of people that can support and guide } \\
\text { me. }\end{array}$ & 5.22 & .928 \\
\hline I put myself in others' situations. & 5.14 & .930 \\
\hline I understand my personal values and beliefs. & 5.42 & .782 \\
\hline I recover from setbacks. & 4.99 & .863 \\
\hline I can be flexible when a change occurs. & 4.95 & .936 \\
\hline I look for new opportunities to improve myself. & 5.38 & .822 \\
\hline I respond calmly in ambiguous situations. & 4.58 & 1.090 \\
\hline
\end{tabular}

(Table 5 Continues) 
(Table 5 Continued)

\begin{tabular}{lcc}
\hline Item & Mean & $\begin{array}{c}\text { Std. } \\
\text { dev. }\end{array}$ \\
\hline I am willing to adapt my perspective to incorporate others' & 5.07 & .839 \\
perspectives when necessary. & 5.33 & .951 \\
I enjoy exploring cultures other than my own. & 5.15 & .873 \\
I maintain productive relationships. & 5.34 & .854 \\
I am empathetic toward others. & 5.10 & .908 \\
I understand my strengths and weaknesses. & 4.92 & 1.029 \\
I remain positive in challenging situations. & 5.08 & .785 \\
I am able to rise again when things don't go the way I hoped. & 5.16 & .916 \\
I expose myself to new experiences. & 5.06 & .917 \\
I role model a positive attitude for others in difficult situations. & & \\
\hline
\end{tabular}

Before exploring the factor structure of the 30 items created for the global leadership assessment, sampling was first examined. The Kaiser-Meyer-Olkin measure of sampling adequacy was .910 , above the commonly recommended value of .7 (Meyers et al., 2006), and Bartlett's test of sphericity was significant $\left(\chi^{2}(435)=4243.925, \mathrm{p}<\right.$ .000), indicating the sample was good. To decide what to retain, Kaiser's (1960) criterion was used, which suggests retaining only those components whose eigenvalues are greater than 1.0. Six components with an eigenvalue of 1.0 or greater explain $61.658 \%$ of the total variance (see Table 6). Additionally, the scree test was consulted and it yielded a similar six components (Figure 2), which was more easily interpreted after collapsing any factors under 3\% (Figure 3) since there were several clustered together close to the bend (Costello \& Osborne, 2005; Yong \& Pearce, 2013). 


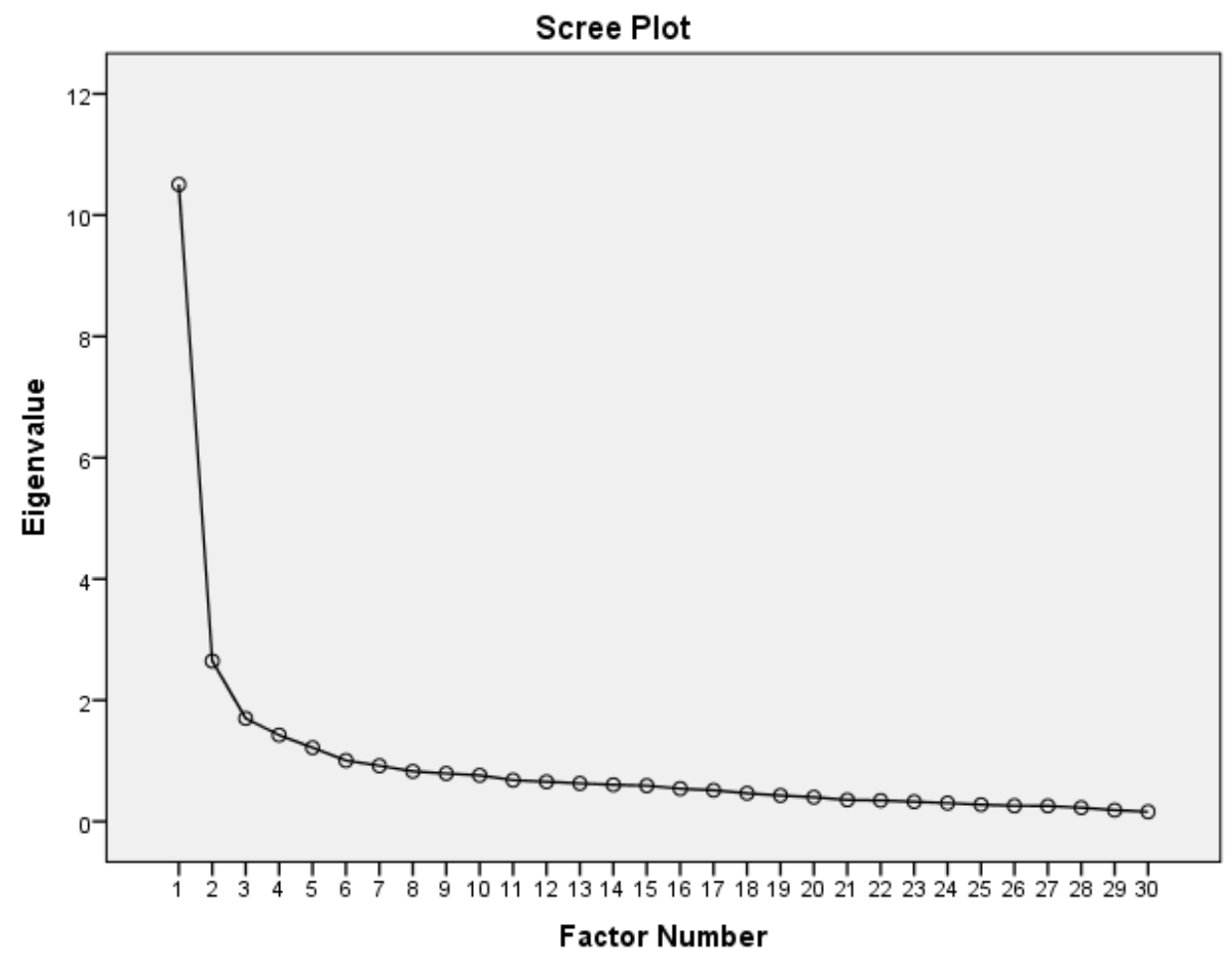

Figure 2. O'Keefe Global Leadership Assessment Scree Plot. This figure illustrates the original scree plot output from SPSS. 


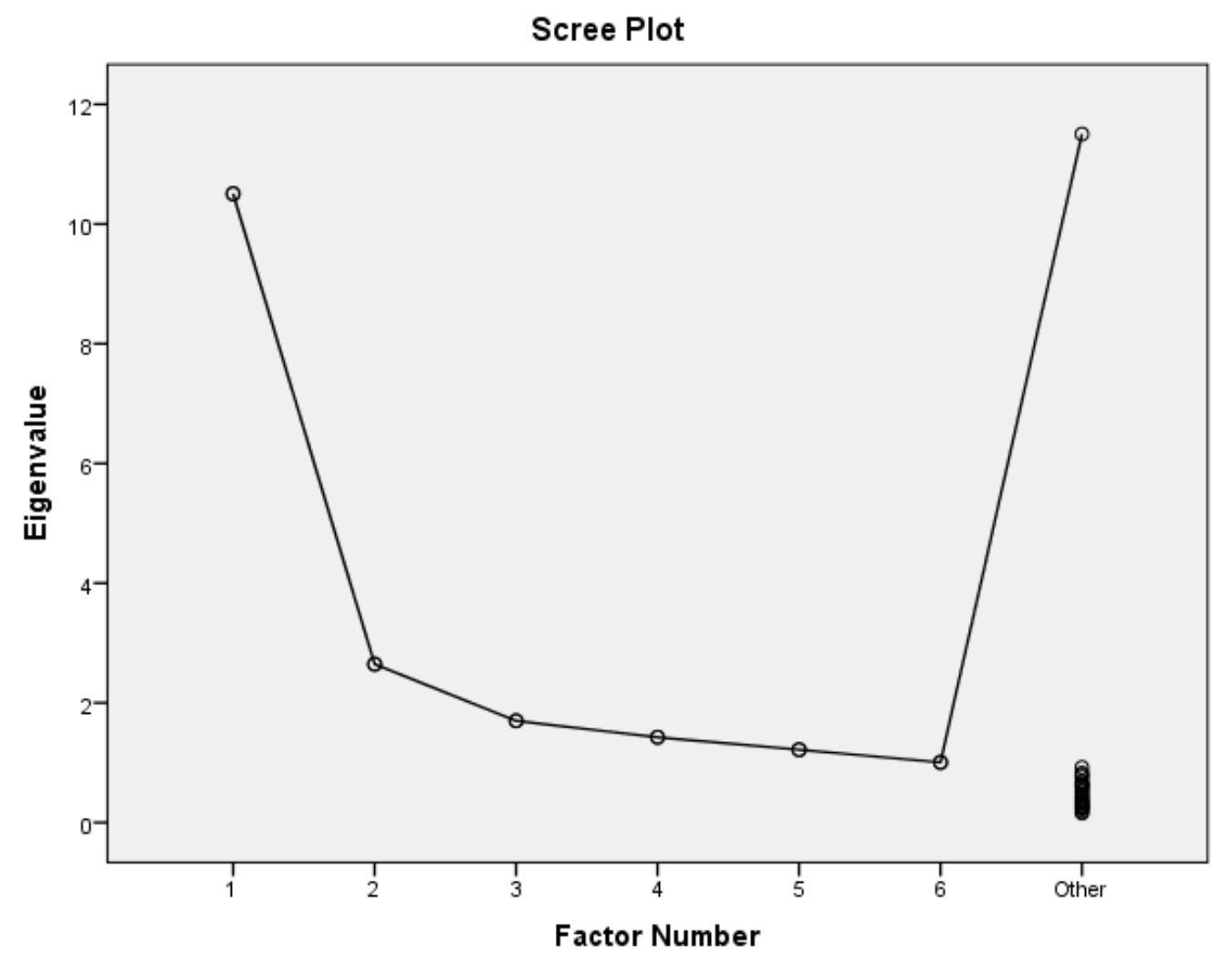

Figure 3. O'Keefe Global Leadership Assessment Collapsed Factors Scree Plot. This figure illustrates the scree plot output from SPSS after collapsing factor categories under $3 \%$.

As presented in Table 6, some items load more neatly than other items on the factors, for instance, productive relationships, empathy, others' perspectives, selfdevelopment, diversity and resiliency have the items primarily load as expected. But other items have some higher cross factor loadings, such as positive attitude, selfunderstanding, responding to change, and responding to ambiguity. The first factor had five items $(1,2,3,4$, and 6$)$ that had primary loadings over .4 with two (items 4 and 6 ) that were cross-loaded. Item 5 is being kept in the first factor, even though it primarily loaded into factor three, because the theory suggests that item is a stronger aspect of factor one than in factor three. The second factor is incorporating nine items (7-15), 
though item 7 did not load into this factor. The researcher tried removing this item and its removal skewed all factor results to be almost uninterpretable. Instead, the researcher is again following the theory when including that item in the second factor. The other eight items primarily load into factor two with item 8 cross-loading into two other factors and items 12,14, and 15 cross-loading into one other factor. The third factor includes six items (16-21). Five of the six items primarily load into this factor though item 17 crossloads into one other factor, item 21 cross-loads into two different factors, and item 18, which does not primarily load into this factor, though theory again suggests it remain in this factor anyway, cross-loads into two other factors. The fourth factor includes three items (22-24) that all primarily load into it with only item 23 cross-loading into another item. The fifth factor also includes three items (25-27). Two of them primarily load here, while item 26 that does not still fit the theory and is cross-loaded to only one other factor. Finally, factor six has three items (28-30) that all primarily load in it, though two of the items (28 and 30) cross-load into two different factors.

The six factors that resulted from the loadings required new labels for three of the underlying constructs discovered, while the other three constructs maintained their originally hypothesized label. The global leadership competency frameworks discussed in the literature review in Chapter 2 provided guidance in naming the domains that emerged (DeVellis, 2016; Reio \& Shuck, 2015). 
Table 6

Summary of Exploratory Factor Analysis Results for O'Keefe Global Leadership Assessment $(N=279)$

\begin{tabular}{|c|c|c|c|c|c|c|c|}
\hline & \multirow[b]{2}{*}{ Item } & \multicolumn{6}{|c|}{ Factor Loadings } \\
\hline & & 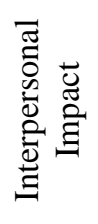 & 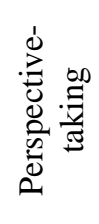 & 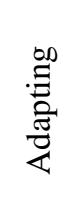 & $\begin{array}{l}\stackrel{\overrightarrow{0}}{0} \\
\frac{0}{0} \\
\stackrel{\overrightarrow{0}}{0}\end{array}$ & 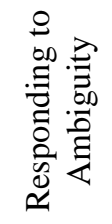 & 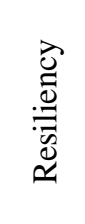 \\
\hline \multirow{3}{*}{ 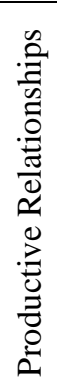 } & $\begin{array}{l}\text { 1. I am willing to take time to } \\
\text { develop productive relationships. }\end{array}$ & .649 & & & & & \\
\hline & $\begin{array}{l}\text { 2. I am building a network of } \\
\text { people that can support and guide } \\
\text { me. }\end{array}$ & .578 & & & & & \\
\hline & $\begin{array}{l}\text { 3. I maintain productive } \\
\text { relationships. }\end{array}$ & .678 & & & & & \\
\hline \multirow{3}{*}{ 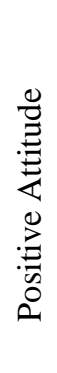 } & $\begin{array}{l}\text { 4. I role model a positive } \\
\text { attitude for others in difficult } \\
\text { situations. }\end{array}$ & .457 & & .440 & & & \\
\hline & $\begin{array}{l}\text { 5. I demonstrate a can-do } \\
\text { attitude. }\end{array}$ & .381 & & .546 & & & \\
\hline & $\begin{array}{l}\text { 6. I remain positive in } \\
\text { challenging situations. }\end{array}$ & .422 & & & & .395 & \\
\hline \multirow{3}{*}{ 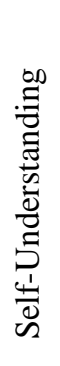 } & $\begin{array}{l}\text { 7. I understand my strengths and } \\
\text { weaknesses. }\end{array}$ & .418 & & & & & .311 \\
\hline & $\begin{array}{l}8 \text {. I understand my personal } \\
\text { values and beliefs. }\end{array}$ & .326 & .335 & .304 & & & \\
\hline & $\begin{array}{l}\text { 9. I am aware of my emotions } \\
\text { during interactions with other } \\
\text { people. }\end{array}$ & & .371 & & & & \\
\hline
\end{tabular}




\begin{tabular}{|c|c|c|c|c|c|c|c|}
\hline & \multirow[b]{2}{*}{ Item } & \multicolumn{6}{|c|}{ Factor Loadings } \\
\hline & & 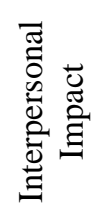 & 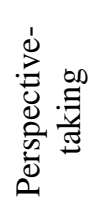 & 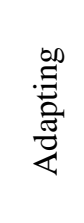 & $\begin{array}{l}\stackrel{\overrightarrow{0}}{\infty} \\
\stackrel{0}{0} \\
\stackrel{\overrightarrow{0}}{\Delta}\end{array}$ & 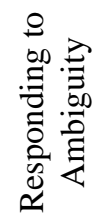 & 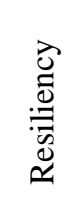 \\
\hline \multirow{3}{*}{ 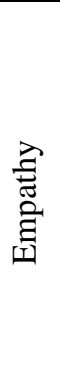 } & $\begin{array}{l}\text { 10. I put myself in others' } \\
\text { situations. }\end{array}$ & & .506 & & & & \\
\hline & $\begin{array}{l}\text { 11. I am empathetic toward } \\
\text { others. }\end{array}$ & & .646 & & & & \\
\hline & $\begin{array}{l}\text { 12. I emotionally connect with } \\
\text { people who have experiences } \\
\text { different from my own. }\end{array}$ & & .330 & & .320 & & \\
\hline \multirow{3}{*}{ 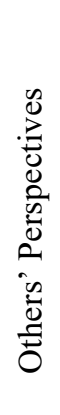 } & $\begin{array}{l}\text { 13. I consider others' } \\
\text { perspectives when making } \\
\text { decisions. }\end{array}$ & & .543 & & & & \\
\hline & $\begin{array}{l}\text { 14. I show interest in others' } \\
\text { perspectives. }\end{array}$ & & .529 & & .357 & & \\
\hline & $\begin{array}{l}\text { 15. I am willing to adapt my } \\
\text { perspective to incorporate others' } \\
\text { perspectives when necessary. }\end{array}$ & & .641 & & & .322 & \\
\hline \multirow{3}{*}{ 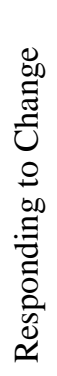 } & $\begin{array}{l}\text { 16. I adjust my behavior when } \\
\text { something occurs that is out of } \\
\text { my control. }\end{array}$ & & & .385 & & & \\
\hline & $\begin{array}{l}\text { 17. I implement a new plan } \\
\text { when a change occurs. }\end{array}$ & & & .525 & & & .438 \\
\hline & $\begin{array}{l}\text { 18. I can be flexible when a } \\
\text { change occurs. }\end{array}$ & & & .334 & & .482 & .425 \\
\hline \multirow{3}{*}{ 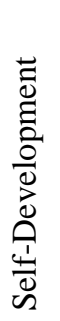 } & $\begin{array}{l}\text { 19. I am committed to life-long } \\
\text { learning. }\end{array}$ & & & .645 & & & \\
\hline & $\begin{array}{l}\text { 20. I look for new opportunities } \\
\text { to improve myself. }\end{array}$ & & & .627 & & & \\
\hline & $\begin{array}{l}\text { 21. I expose myself to new } \\
\text { experiences. }\end{array}$ & .316 & & .360 & .306 & & \\
\hline
\end{tabular}




\begin{tabular}{|c|c|c|c|c|c|c|c|}
\hline & \multirow[b]{2}{*}{ Item } & \multicolumn{6}{|c|}{ Factor Loadings } \\
\hline & & 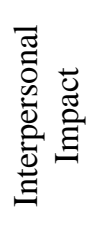 & 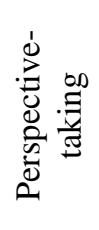 & 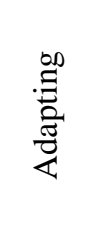 & 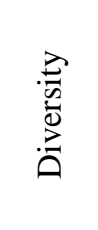 & 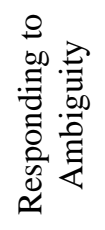 & 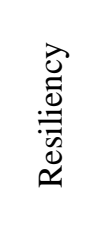 \\
\hline \multirow{3}{*}{ 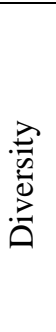 } & $\begin{array}{l}\text { 22. I am curious about different } \\
\text { cultures. }\end{array}$ & & & & .840 & & \\
\hline & $\begin{array}{l}\text { 23. I appreciate the differences } \\
\text { of other cultures. }\end{array}$ & & .364 & & .666 & & \\
\hline & $\begin{array}{l}\text { 24. I enjoy exploring cultures } \\
\text { other than my own. }\end{array}$ & & & & .865 & & \\
\hline \multirow{3}{*}{ 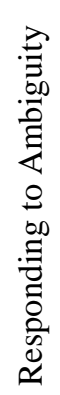 } & $\begin{array}{l}25 \text {. I react comfortably to } \\
\text { uncertainty. }\end{array}$ & & & & & .691 & \\
\hline & $\begin{array}{l}\text { 26. I remain open to new } \\
\text { information } \\
\text { in uncertain situations. }\end{array}$ & & .336 & & & .302 & \\
\hline & $\begin{array}{l}\text { 27. I respond calmly in } \\
\text { ambiguous situations. }\end{array}$ & & & & & .692 & \\
\hline \multirow{6}{*}{ 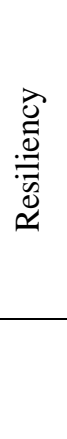 } & $\begin{array}{l}\text { 28. I quickly bounce back from } \\
\text { failures. }\end{array}$ & & & & & .400 & .532 \\
\hline & 29. I recover from setbacks. & & & & & & .747 \\
\hline & $\begin{array}{l}\text { 30. I am able to rise again when } \\
\text { things don't go the way I hoped. }\end{array}$ & .364 & & & & & .511 \\
\hline & Eigenvalues & 10.51 & 2.65 & 1.70 & 1.42 & 1.22 & 1.01 \\
\hline & $\%$ of variance & 9.97 & 9.97 & 9.32 & 8.56 & 7.81 & 7.49 \\
\hline & Cumulative \% & 9.96 & 19.65 & 28.98 & 37.54 & 45.35 & 52.84 \\
\hline
\end{tabular}

Note: Factor loadings <.30 are suppressed. 
The Six Topic Factors. Six items (1-6) loaded onto factor one labeled "Interpersonal Impact." The hypothesized competencies for those items were "Productive Relationships" and "Positive Attitude." When referring to the global leadership literature, it was discovered that several of the theories cited for these competencies in the analysis included the word or concept of "interpersonal" (Bird, 2013; Bird \& Osland, 2004; Javidan \& Teagarden, 2011; Javidan \& Walker, 2013; Mendenhall \& Osland, 2002; Stevens, et al., 2014; Van Der Zee \& Van Oudenhoven, 2000). As the researcher reread the global leadership definitions in each category, it was also recognized that when discussing optimism, it was the "impact" optimism had on the group that was being referenced (Javidan \& Teagarden, 2011; Jokinen, 2005; Sinicrope, et al., 2007; Stevens, et al., 2014). Therefore, the label "Interpersonal Impact" was created. The items included under this title measure how a leader's positive outlook and actions create the ability to develop strong productive relationships.

Nine items (7-15) are included in factor two labeled "Perspective-taking," which includes the hypothesized competencies Self-Understanding, Empathy, and Others' Perspectives. In the global leadership literature awareness was a base used in all three competencies although the focus may have differed the definitions did not stop at just awareness per se but was described variously as awareness of self, awareness of emotions, awareness of others, and awareness of perspectives. The literature showed how awareness should be used to be most effective - by starting with these types of awareness's and then to aid in leadership (Bird, 2013; Javidan \& Teagarden, 2011; Javidan \& Walker, 2013; Jokinen, 2005; Koester \& Olebe, 1988; Matsumoto \& Hwang, 2013; Sinicrope, et al., 2007; Stevens, et al., 2014; Van Der Zee \& Van Oudenhoven, 
2000; Van Dyne, et al., 2008). Therefore, "Perspective-Taking" was appropriated as the most appropriate label, because a leader must first be aware of where they stand on an issue (Self-Understanding), be conscious of how others may be feeling regarding that issue (Empathy), then take the time to understand perspectives different from their own prior to deciding on the issue (Others' Perspectives).

Six items (16-21) loaded onto factor three labeled "Adapting." The hypothesized competencies for those items were Responding to Change and Self-Development. The global leadership definitions associated with these two competencies discuss the ability to be open to new information, though the type of information differed, and then adapt as necessary (Bird, 2013; Bird \& Osland, 2004; Javidan \& Teagarden, 2011; Jokinen, 2005; Koester \& Olebe, 1988; Matsumoto \& Hwang, 2013; Mendenhall \& Osland, 2002; Sinicrope, et al., 2007; Stevens, et al., 2014; Van Der Zee \& Van Oudenhoven, 2000; Van Dyne, et al., 2008).

Three items (22-24) loaded onto factor four, Diversity, which is the first of the three factors that maintained the hypothesized definition. Three items (25-27) loaded onto factor five, Responding to Ambiguity, another that fit its hypothesized definition. Finally, three items (28-30) also loaded onto factor six, Resiliency, the last of the hypothesized definitions that were kept.

Reliability for each of the new components was also examined using Cronbach's alpha. The alphas were good: .829 for Interpersonal Impact (6 items), .835 for Perspective-taking ( 9 items), .803 for Adapting (6 items), .878 for Diversity (3 items), .664 for Responding to Ambiguity ( 3 items), and .815 for Resiliency ( 3 items). The reliability for the instrument was high (30 items; $\alpha=.932$ ) (Meyers et al., 2006). 


\section{Summary}

Chapter 4 presented an explanation of how the results of each phase were used in the next phase of research. It also presented the qualitative and quantitative methods used to determine the results of each phase. Phase one was a qualitative phase that utilized 13 subject matter experts to determine the global leadership framework mapped within the Student Leadership competencies. It also established validity evidence based on test content through those same experts matching items to competencies in a table of specifications. Phase two was also a qualitative phase that established validity evidence based on cognitive processes through cognitive interviews. Phase three was a pilot of the quantitative phase of the study, which resulted in three items being removed through use of phase two data. The fourth and final phase was a quantitative phase that established validity evidence based on internal structure and reliability evidence with Cronbach's alpha. 


\section{CHAPTER V}

\section{DISCUSSION}

Chapter 5 begins with a summary of the study, including a presentation of answers to each of the study's research questions. The chapter continues with an interpretation and analysis of the results as they relate to the theoretical framework and existing literature. The chapter concludes with study limitations, implications for practice, and recommendations for future research.

\section{Summary of the Study}

For decades, institutions of higher education across the United States have offered co-curricular activities that help students develop leadership capabilities (Astin \& Astin, 2000; Hamrick et al, 2002; National Association of Student Personnel Administrators, 2016). As the need for global leaders increases, employers are expressing a skill crisis whereby graduates are entering the workforce without the appropriate leadership skills for a global environment (Bersin, 2012; Elmore, 2013; Ficsher, 2015; Gillis, 2011; Zenger et al., 2014). As established in Learning Reconsidered, students do not simply absorb material presented to them; they need to be engaged with the material to integrate it into their personal development (Keeling, 2004). Co-curricular activities are essential to help students develop the skills necessary for global leadership.

One of the greatest challenges for student affairs, the field that enhances student growth and development through co-curricular programs, is funding (Sandeen \& Barr, 2014). Consequently, creating a new program that specifically focuses on global leadership competencies may not be feasible for most institutions. To identify learning outcomes for their current co-curricular programs many institutions are using Seemiller's 
Student Leadership Competencies (Seemiller \& O'Keefe, 2016). However, the global aspects of leadership were not yet acknowledged by the existing Student Leadership Competencies research. Self-assessments are often used in leadership development programs not because they are great predictors of leadership ability (John \& Robins, 1993), but because their results facilitate self-reflection. Self-reflection leads to better self-awareness, which allows people to better employ their strengths and improve their weaknesses (Drucker, 2005; Moore et al., 1997; Luft \& Ingham, 1961; Pearman, 1999; Shertzer \& Doyle, 2006; Tjan, 2012; Travers et al., 2015; Zimmerman-Oster \& Burkhardt, 2000). A literature review on global leadership showed that the global leadership assessments currently available were developed for corporations to use as training and promotional tools for their top managers, not to provide self-awareness to college students (Bird \& Stevens, 2013).

This study aimed to combine the concepts of global leadership and the Student Leadership Competencies so that students would have opportunities to develop global leadership competencies on their own. Specifically, it created a global leadership competencies self-assessment instrument mapped within the Student Leadership Competencies, then demonstrated the extent to which the instrument yields evidence that supports valid and reliable inferences about students' global leadership competencies. This study addressed the following research questions:

1. Does the Global Leadership Competencies self-assessment instrument yield valid inferences about students' global leadership competencies?

2. Does the Global Leadership Competencies self-assessment instrument yield reliable inferences about students' global leadership competencies? 


\section{Methods}

The study used an exploratory sequential mixed methods design, which is a mixed methods procedure where qualitative data are collected in early phases of a study; then, findings from the qualitative data are used for the following quantitative data phase(s) (Teddlie \& Tashakkori, 2006). There were four different phases of research.

\section{Phase One}

Validity evidence based on test content was established through utilizing the specialized knowledge of 13 subject matter experts (AERA, 2014). The subject matter experts were chosen based on their knowledge of the Student Leadership Competencies (2015), global leadership, practical application of leadership programming, a combination of those, or a related focus area. These experts analyzed two different tables of specifications. The first compared the global leadership research against the Student Leadership Competencies definitions (Seemiller, 2013), which created a global leadership framework mapped within the Student Leadership Competencies. The second was a random list of potential items for the instrument, to be matched with the definitions of the competencies in the newly established global leadership competencies framework. In order to be included on the instrument during the next phase of research, items had to be correctly matched to the intended competency by at least three-fourths of the experts.

\section{Phase Two}

Validity evidence based on cognitive response processes was established through cognitive interviews (AERA, 2014). These interviews were conducted with ten FIU students to help determine understanding and clarity of each of the items on the instrument. The students were recruited from varying majors with an effort to reflect the 
demographics of the population with respect to gender, race, and ethnicity according to the National Center for Educational Statistics. The race/ethnicity breakdown of the participants was five White, two Hispanic, two Black, and one Asian. The gender breakdown was four males and six females. The participants took the instrument from the Qualtrics platform on an iPad in a closed-door office setting with two researchers present. They were asked to use a think-aloud process as they went through the instrument and to give examples that helped them decide how to rate themselves. The researchers looked for clarity of the behavioral statements, appropriateness of the statements for the population, if the language/terminology was understandable, if the questions were appropriate for the scope of the instrument, and if the information being asked was easily accessible in the students' thought processes (Willis, 2005). A few wording adjustments were made before moving on to the next phase of research.

\section{Phase Three}

A pilot of the instrument was then conducted with a convenience sample of 78 FIU students. The data collected from this pilot was intended to help determine the feasibility of the larger study (Babbie, 1990; DeVellis, 2016). An exploratory factor analysis with a varimax rotation was ran in SPSS. Some dimensionality emerged, indicating that there were underlying common factors to uncover, so it was decided to

move on to the next phase of research. Four items were removed using data from phase two after no item reduction emerged from the statistical analysis during this phase (Costello \& Osborne, 2005; Henson \& Roberts, 2006; Yong \& Pearce, 2013). 


\section{Phase Four}

Validity evidence based on internal structure and evidence of reliability were established during this phase. (AERA, 2014). The final sample was recruited by utilizing a snowballing technique, which resulted in a sample of 279 undergraduate students from 30 different institutions of higher education across the USA representing approximately 145 different majors. Participants could choose as many of the race demographics as appropriate, which provided a breakdown of 214 White, 53 Black or African American, 4 American Indian or Alaska Native, 20 Asian, 3 Native Hawaiian or Pacific Islander, and 28 Other. Additionally, 136 also identified as Spanish, Hispanic, or Latino. The gender breakdown was 78 males, 200 females, and 1 other. For age ranges 3 were under 21; 245 were 18-24; 20 were 25-34; and 11 were over 35 . An exploratory factor analysis using a principle axis factoring extraction with a varimax rotation was conducted in SPSS. Kaiser-Meyer-Olkin and Bartlett's test of sphericity were used to examine sampling adequacy. In the decision of which factors to keep, eigenvalue of 1.0 or greater, total variance, and a scree test were used. The global leadership research was consulted to name three of the six factors that emerged and the hypothesized names were kept for the other three factors. Cronbach's alpha was used to establish reliability on the instrument and each of the six factors.

\section{Results}

\section{Research Question 1}

Evidence supported the finding that the Global Leadership Competencies selfassessment instrument yielded valid inferences about students' global leadership competencies. Validity evidence based on content was established through the use of 13 
subject matter experts. Validity evidence based on a cognitive process was established through cognitive interviews. Finally, validity evidence based on internal structure was established by conducting an exploratory factor analysis. Specifically, an exploratory factor analysis using a principal axis factoring extraction with a varimax rotation was conducted in SPSS on data gathered from 279 participants. The Kaiser-Meyer-Olkin measure of sampling adequacy was .910 , above the commonly recommended value of .7 (Meyers et al., 2006), and Bartlett's test of sphericity was significant $\left(\chi^{2}(435)=\right.$ $4243.925, \mathrm{p}<.000)$. Six components with an eigenvalue of 1.0 or greater explained $61.658 \%$ of the total variance, which was also verified with a scree test. The results of the loadings from the principal axis factoring for the six factors required new labels for three of the underlying constructs discovered, while the other three constructs maintained their originally hypothesized label.

\section{Research Question 2}

Evidence supported the finding that the Global Leadership Competencies selfassessment instrument yielded reliable inferences about students' global leadership competencies (30 items; $\alpha=.932$ ). Reliability for each of the scales was also examined using Cronbach's alpha: .829 for Interpersonal Impact (6 items), .835 for Perspectivetaking (9 items), .803 for Adapting (6 items), .878 for Diversity (3 items), .664 for Responding to Ambiguity (3 items), and .815 for Resiliency (3 items).

\section{Interpretation and Analysis of Results}

This study has provided a tool to help facilitate a student's out-of-the-classroom or co-curricular education specifically related to developing global leadership competencies. The study's methodology was based on an integrated concept of validity 
and reliability, where test content, cognitive response process, internal structure, and reliability were used as sources of evidence regarding the interpretation and use of the results from the global leadership assessment instrument (AERA, 2014; Messick, 1996). The combined results from all four research phases of the study provided evidence that the instrument yields valid and reliable inferences about students' global leadership competencies.

Internationalizing the co-curriculum is just as important as internationalizing the curriculum as educators try to address the global leadership skill crisis being expressed by employers (ACE, 2013; Bersin, 2012; Commission on International Education, 1998; Elmore, 2013; Ficsher, 2015; Gacel-Ávila, 2005; Gibson et al., 2008; Gillis, 2011; Global Learning, 2015; NAFSA, 2016; Grudzinski-Hall, 2007; Landorf \& Doscher, 2015; Zenger et al., 2014). The instrument created by the study aids students in identifying cocurricular activities appropriate for their personal global leadership development needs. Using the results of the test will allow students to understand their level of proficiency of global leadership competencies throughout their educational development and better employ their strengths and improve their weaknesses from the self-awareness created by their results (Drucker, 2005; Moore et al., 1997; Luft \& Ingham, 1961; Pearman, 1999; Shertzer \& Doyle, 2006; Tjan, 2012; Travers et al., 2015; Zimmerman-Oster \& Burkhardt, 2000).

It is important to note that because the global leadership competency instrument has the terminology currently being used by leadership development programs in university settings - the Student Leadership Competencies (Seemiller, 2013), students can use their results from this instrument and using the SLC put together their own global 
leadership competency development plan. This is particularly relevant if their university is unable to offer dedicated global leadership competency development programming for them. And, even if their university does not use the Student Leadership Competencies for learning outcomes, there are still online resources on these competencies easily searchable and accessible for students (Seemiller, 2017). The competency labels are also intuitive and simple enough that students could also find developmental resources without formal reference to the Student Leadership Competencies.

While there may not be an agreed upon definition of global leadership, the concept is still widely realized and actualized in today's societies throughout the world (Blaess et al., 2012; Jokinen, 2005; Mendenhall et al., 2012; Mendenhall \& Osland, 2002; Osland et al., 2006; Rhinesmith, 1993). Institutions of higher education need to prepare students for global leadership situations such as working in different cultures; combining business practices to fit multinational needs; cultivating trust among team members that may not be of the same nationality and/or only work with each other remotely; overcoming communication barriers; creating clarity in team objectives where values may differ, dealing with different laws and regulations; overcoming stereotypes and prejudices; and being able to manage through the complex, changing, and often ambiguous global environment (Caligiuri, 2006; Danielsson, 2015; Govindarajan \& Gupta, 2001; Hassanzadeh et al., 2015; Holt, 2015; Levy et al., 2007; Taneja et al., 2015; Voronchenko et al., 2015). The instrument created in this study is designed to be a starting point for students on their global leadership competency development journey. 


\section{Study Limitations}

There is an inherent subjectivity in the decisions necessary to conduct an exploratory factor analysis; consequently, other researchers may have made different choices. Generalizability of this study to the larger population was limited by the demographic characteristics of the study's convenience samples during the cognitive interviews in phase two and data collection during phase four.

\section{Implications for Theory}

This study was focused on determining measurable global leadership competencies that could be achieved or learned by a person, which then provided the content for the resulting instrument. The study derived its theoretical foundation from constructivism, which guided its approach to analysis of the global leadership competencies literature and research methods. The idea of striving for a selftransforming mind, where individuals see beyond themselves, others, and the systems of which they are a part, to form an understanding of how all people and systems interconnect, provided guidance when analyzing the global leadership literature and when creating the self-assessment instrument intended to be used a self-reflection, which leads to better self-awareness (Drucker, 2005; Moore et al., 1997; Kegan, 1994; Luft \& Ingham, 1961; Pearman, 1999; Shertzer \& Doyle, 2006; Tjan, 2012; Travers et al., 2015; Zimmerman-Oster \& Burkhardt, 2000). A constructivist approach was used in both phase one, when the subject matter experts provided guidance on the test content validity, and in phase two during the cognitive interviews when participants provided different experiences to answer the questions. Both groups - subject matter experts and cognitive interview participants - provided their own understanding and knowledge based on their 
experience with the global leadership content and reflection on those experiences, which was incorporated into the design of the instrument.

Additionally, the construct of "Adapting", which combined the originally hypothesized competencies of Responding to Change and Self-Development, was explained as global leaders needing the ability to be open to new information and then adapt as necessary. This confirms the continual adaptation described in constructivism where humans generate knowledge and meaning by reflecting on experience (Kegan, 1994).

The instrument that was developed in this study can also be used to support global education theory. In Attaining a Global Perspective, a seminal article in global education written in 1982, author Robert Hanvey set forth a suite of five capacities that individuals can develop in their lives to reach a global perspective, one of these capacities, the linchpin of the others, is "perspective consciousness," which Hanvey defined as, the recognition or awareness on the part of the individual that he or she has a view of the world that is not universally shared, that this view of the world has been and continues to be shaped by influences that often escape conscious detection, and that others have views of the world that are profoundly different from one's own. (p. 162)

Although this article was not covered in the literature review in this study, the construct "Perspective-Taking," can be found as a combination of three of the originally hypothesized competencies of the study, Self-Understanding, Empathy, and Others' Perspectives. Together, these three competencies make up perspective consciousness, supporting Hanvey's definition. 


\section{Implications for Practice}

This study resulted in an instrument that can be utilized by undergraduate students at institutions of higher education in the USA to help them assess and reflect on their global leadership competencies (See Appendix W) and more specifically in relation to a student's out-of-the-classroom or co-curricular education.

The study adds to the research literature on global leadership competencies with the identification of a global leadership framework mapped within the Student Leadership Competencies. The Student Leadership Competencies are now being used by institutions of higher education across the United States to help connect out-of-classroom activities to learning outcomes that resonate across all academic disciplines; creating a common language of leadership for employers, academics, student affairs professionals, and students (Seemiller, 2016). This study created a global leadership framework for the Student Leadership Competencies - Responding to Change, Self-Development, Responding to Ambiguity, Others' Perspectives, Diversity, Productive Relationships, Empathy, Self-Understanding, Positive Attitude, and Resiliency, which are the underlying competencies required for a person desiring to be a global leader.

Additionally, through the internal structure validation process three underlying constructs that combined some of the competencies were uncovered. The study shows that to improve on Interpersonal Impact a student should look for activities that develop both Productive Relationships and Positive Attitude. If a student desires to increase their Perspective-taking, they should look for activities that include Self-Understanding, Empathy, and Others' Perspectives. And, finally, if they want to work on Adapting, they should look for both Responding to Change and Self-Development activities. The 
constructs that emerged in this study suggest programs that address these competency combos should be developed by global leadership educators.

This study could also apply to another body of research on competencies that many practitioners in higher education are focusing on - the National Association for College Employers (NACE) Career Readiness Competencies. The NACE Career Readiness Competencies were created by a task force of college career services and $\mathrm{HR} /$ staffing professionals that conducted extensive research among employers to identify competencies associated with career readiness. The eighth competency Global/Intercultural Fluency - is defined as "Value, respect, and learn from diverse cultures, races, ages, genders, sexual orientations, and religions. The individual demonstrates, openness, inclusiveness, sensitivity, and the ability to interact respectfully with all people and understand individuals' differences" (NACE, 2017). The instrument created in the study can be utilized as a pre / post assessment of that competency and the percent change calculated from those administrations can help to demonstrate the impact a program may be having toward that competency.

Other researchers interested in global leadership competencies can also use this tool in a similar way. For example, human resource professionals could utilize the instrument with expatriates. In addition to using the instrument as a direct research tool, the data being collected as individuals utilize the instrument will be a databank that can be used for future research.

In summary, students, student affairs practitioners, leadership educators, researchers interested in global leadership, and employers can all benefit from the results of this study. Students now have a tool that facilitates self-reflection on global leadership 
competencies. Student affairs practitioners benefit because they can offer this instrument as a resource to their students to assist them in developing global leadership competencies without any additional resources and programs. Leadership educators can develop programs related to the combined competency constructs. Global leadership researchers have a tool for direct measurement and a potential databank. Finally, employers benefit from the results of the study because more students will graduate with the necessary underlying global leadership competency skills as the global leadership framework and instrument are utilized.

\section{Recommendations for Future Research}

Additional research is recommended on this instrument as establishing validity is a process (Messick, 1989). It is recommended that a Confirmatory Factor Analysis (CFA) be conducted on the instrument to test the six factors uncovered during the exploratory factor analysis in this study (Costello \& Osborne, 2005; Fabrigar, et al., 1999; Henson \& Roberts, 2006; Yong \& Pearce, 2013; Williams, et al., 2010). CFAs are "used to test theory when the analyst has sufficiently strong rationale regarding what factors should be in the data and what variables should define each factor" (Henson \& Roberts, 2006).

Expanding the research to other populations is recommended. During the bulk data collection during phase four before the subset of undergraduate students was extracted for this study's use, graduate students and professional staff members were included, which allowed the researcher to run some preliminary exploratory factor analyses for those populations. Communalities in constructs emerged for the different 
groups and the groups combined, but the competencies of Self-Understanding and SelfDevelopment did not load the same in every sample population.

It may also be valuable to run additional exploratory factor analyses with a larger sample without those two competencies (Self-Understanding and Self-Development) to examine the effect that has on the loadings for those different populations. Theoretically, if a person is choosing to take a global leadership competencies self-assessment, they are already demonstrating that they have a desire to develop themselves and know the need to also understand themselves. So, those competencies may not be essential to include in the framework in different populations.

Utilizing qualitative techniques to help explain why Self-Understanding and SelfDevelopment were not consistent in all populations is another option for future research. The varying levels of experience with global leadership in the other populations may explain why the quantitative sample from the study was unclear. Targeting graduate students in a major with a global focus versus graduate students in a non-global focused major and comparing their results from the instrument then interviewing them regarding their results is an example of a study that may provide this understanding.

Further research can also be conducted with the data collected from the instrument being utilized. Multivariate analysis of the global leadership competencies and gender, race, and higher education institutions, as well as other statistical analyses may uncover useful insights. Patterns from these analyses may emerge that will continue to inform the global leadership research. 


\section{Conclusions}

As colleges and universities across the United States continue to help their students become career ready, global leadership is an area of competence that employers have identified as essential (NACE, 2017). Although they may have limited resources, universities still need to be able to provide students opportunities to develop their global leadership competencies. Co-curricular activities that use the Student Leadership Competencies (Seemiller, 2013) as learning outcomes combined with the global leadership competencies instrument that was a result of this study provide students an opportunity to be proactive in their own global leadership competency development. The instrument created in this study was designed to provide self-awareness of a student's proficiency in the various global leadership competencies. After receiving their results from this instrument students are then able to seek out opportunities available either on their campus or in other out-of-the-classroom activities to grow or enhance their competencies in different aspects of global leadership. Using this instrument could significantly contribute to the beginning of their journey as global leaders. 


\section{REFERENCES}

ACE. (2013, December). Internationalization in Action: Internationalizing the Curriculum, Part 1 - Individual Courses. Retrieved from https://www.acenet.edu/news-room/Pages/Intlz-in-Action-2013-December.aspx

American Council on Education. Center for Institutional, \& International Initiatives. (2002). Beyond September eleventh: A comprehensive national policy on international education. Washington, DC: American Council on Education, Center for Institutional and International Initiatives.

American Educational Research Association (AERA), American Psychological Association (APA), \& National Council on Measurement in Education (NCME). (2014). Standards for educational and psychological testing. Washington, DC: American Educational Research Association.

Aperian Global. (2016). Cultural competence assessments and surveys. Retrieved from http://www.aperianglobal.com/learning-solutions/assessments-surveys/

Association of American Colleges and Universities [AAC\&U] (2007). Global learning for the new global century: Executive summary with findings from employer survey. Washington, DC: Association of American Colleges and Universities.

Astin, A. W., \& Astin, H. S. (2000). Leadership reconsidered: Engaging higher education in social change. Battle Creek, MI: Kellogg Foundation.

Babbie, E. R. (1990). Survey research methods ( $2^{\text {nd }}$ ed.). Belmont, CA: Wadsworth Publishing Company.

Bass, B. M. (1990). Bass and Stogdill's handbook of leadership: Theory, research and managerial applications ( $3^{\text {rd }}$ ed.). New York, NY: Free Press.

Basseches, M. (1986). Dialectical thinking and young adult cognitive development. In R. A. Mines \& K. S. Kitchener (Eds.), Adult cognitive development: Methods and models (pp. 33-56). New York, NY: Praeger.

Baxter Magolda, M. B. (1992). Knowing and reasoning in college: Gender-related patterns in students' intellectual development. San Francisco, CA: Jossey-Bass.

Belenky, M. F., Clinchy, B. M., Goldberger, N. R., \& Tarule, J. M. (1997). Women's ways of knowing: The development of self, voice, and mind. New York, NY: Basic Books.

Bersin, J. (2012, December 10). Growing gap between what business needs and what education provides. Retrieved from http://www.forbes.com/sites/joshbersin/2012/12/10/growing-gap-between-whatbusiness-needs-and-what-education-provides/\#2b6d7d8d76bf 
Bird, A. (2013). Mapping the content domain of global leadership competencies. Global leadership: Research, practice, and development, 80-96. New York, NY:

Routledge.

Bird, A., \& Osland, J. (2004). Global competencies: An introduction. In H. Lane, J. McNett, M. Mendenhall \& M. Maznevski (Eds.), The Blackwell handbook of global management: A guide to managing complexity (pp. 57-80). Malden, MA: Blackwell Publishing.

Bird, A. \& Stevens, M. (2013). Assessing global leadership competencies. Global leadership: Research, practice, and development, (pp. 113-140) New York, NY: Routledge.

Blaess, D., Hollywood, K., \& Grant, C. (2012). Preparing the professoriate to prepare globally competent leaders. Journal of Leadership Studies, 6, 88-94.

Black, J., Morrison, A., \& Gregersen, H. (1999). Global explorers: the next generation of leaders. New York, NY: Routledge.

Beechler, S., Sondergaard, M., Miller, E.L., \& Bird, A. (2004). Boundary spanning. In H. Lane, M. Maznevksi, M.E. Mendenhall \& J. McNett (Eds.), The handbook of global management: A guide to managing complexity (pp. 121-133). Oxford, UK: Blackwell.

Brake, T. (1997). The global leader: Critical factors for creating the world class organization. Chicago, IL: Irwin Professional Pub.

Braskamp, L., Braskamp, D., \& Engberg, M. (2014, August). Global Perspective Inventory (GPI): Its Purpose, Construction, Potential Uses, and Psychometric Characteristics. Retrieved from http://www.gpi.hs.iastate.edu/documents/BraskampBraskampEngberg2014GPIPs ychometrics.pdf

Caligiuri, P. (2006). Developing global leaders. Human Resource Management Review, $16,219-228$.

Chase-Dunn, C. (1999). Globalization: A world-systems perspective. Journal of WorldSystems Research, 2, 187-215.

Cole, K. (2003). Globalization: Understanding complexity. Progress in Development Studies, 3, 323.

Commission on International Education. (1998). Educating for global competence: America's passport to the future. Washington, DC: Author. 
Costello, A. B., \& Osborne, J. W. (2005). Best practices in exploratory factor analysis: Four recommendations for getting the most from your analysis. Practical assessment, research \& evaluation, 10(7), 1-9.

Creswell, J. W., \& Plano Clark, V. L. (2011). Choosing a mixed methods design. Designing and conducting mixed methods research, 53-106.

Danielsson, L. (2015). Leadership in international projects: A study of the cultural dimension. (Master thesis, DiVA).

DeVellis, R. F. (2016). Scale development: Theory and application (Vol. 26). Newbury Park, CA: Sage.

Drucker, P. F. (2005). Managing oneself. Harvard Business Review, 83, 100-109.

Elmore, T. (2013, February 12). One big way to bridge the gap between education \& employment. Retrieved from http://growingleaders.com/blog/bridge-gapbetween-education-and-employment/

Fabrigar, L. R., Wegener, D. T., MacCallum, R. C., \& Strahan, E. J. (1999). Evaluating the use of exploratory factor analysis in psychological research. Psychological methods, 4(3), 272.

Fischer, K. (2015, May 29). A global education opens doors but leaves many shut out. Retrieved from http://chronicle.com/article/A-Global-EducationOpens/230511/?cid=at

Fisher, D., Rooke, D., \& Torbert, B. (2000). Personal and organisational transformations: Through action inquiry. Boston, MA: Edge/Work Press.

Fraenkel, J. R. \& Wallen, N. E. (2006). How to design and evaluate research in education (Vol. 6). New York, NY: McGraw-Hill.

Gacel-Ávila, J. (2005). The internationalisation of higher education: A paradigm for global citizenry. Journal of Studies in International Education, 9, 121-136.

Gallup. (2016). StrengthsQuest. Retrieved from http://www.strengthsquest.com/content/141728/index.aspx

Gehrke, S. (2006). "Student learning in leadership programs." In Komives, S. R., Dugan, J. P., Owen, J. E., Slack, C., \& Wagner, W. (2011). The handbook for student leadership development. Hoboken, NJ: John Wiley \& Sons.

Gehlbach, H., \& Brinkworth, M. E. (2011). Measure twice, cut down error: A process for enhancing the validity of survey scales. Review of General Psychology, 15(4), 380 . 
Ghasabeh, M. S., Soosay, C., \& Reaiche, C. (2015). The emerging role of transformational leadership. The Journal of Developing Areas, 49, 459-467.

Gibson, K., Rimmington, G., \& Landwehr-Brown, M. (2008). Developing global awareness and responsible world citizenship with global learning. Roeper Review, $30,11-23$.

Gillis, J. (2011). Global leadership development: An analysis of talent management, company types and job functions, personality traits and competencies, and learning and development methods. (Doctoral dissertation, University of Pennsylvania).

Global learning. (2015). Retrieved from http://goglobal.fiu.edu

Global Leadership Excellence, LLC. (2016). Global competence aptitude assessment: EDUCATION. Retrieved from http://www.globallycompetent.com/education.GCAA.htm

Goldsmith, M., Greenberg, C., Robertson, A., \& Hu-Chan, M. (2003). Global leadership: The next generation. Upper Saddle River, NJ: Prentice-Hall.

Govindarajan, V., \& Gupta, A. K. (2001). Building an effective global business team. MIT Sloan Management Review, 42, 63.

Groves, R. M., Fowler Jr, F. J., Couper, M. P., Lepkowski, J. M., Singer, E., \& Tourangeau, R. (2011). Survey methodology (Vol. 2). Hoboken, NJ: John Wiley $\&$ Sons.

Grudzinski-Hall, M. N. (2007). How do college and university undergraduate level global citizenship programs advance the development and experiences of global competencies? (Doctoral dissertation, Drexel University).

Hamrick, F. A., Evans, N. J., \& Schuh, J. H. (2002). Foundations of student affairs practice: How philosophy, theory, and research strengthen educational outcomes. Hoboken, NJ: John Wiley \& Sons.

Hanvey, R. G. (1982). An attainable global perspective. Theory into practice, 21(3), 162167.

Harvey, M. \& Novicevic, M. M. (2004). The development of political skill and political capital by global leaders through global assignments. International Journal of Human Resource Management, 15, 11173-1188.

Hassanzadeh, M., Silong, A. D., Asmuni, A., \& Wahat, N. W. A. (2015). Global leadership and diversity. Journal of Educational and Social Research, 5, 161. 
Henson, R. K., \& Roberts, J. K. (2006). Use of exploratory factor analysis in published research: Common errors and some comment on improved practice. Educational and Psychological measurement, 66(3), 393-416.

Holt, S. (2015). The role of emotional intelligence in global leadership. In Global Enterprise Management (pp. 121-137). New York, NY: Palgrave Macmillan US.

Hu, L., \& Bentler, P. M. (1999). Cutoff Criteria for Fit Indices in Covariance Structure Analysis: Conventional Criteria versus New Alternatives. Structural Equation Modeling, 6(1), 1-55.

Hubley, A. M., \& Zumbo, B. D. (1996). A dialectic on validity: Where we have been and where we are going. The Journal of General Psychology, 123, 207-215.

Hunter, W. D., White, G. P., \& Godbey, G, C. (2006). What does it mean to be globally competent? Journal of Studies in International Education, 10, 267-285.

Javidan, M., Hough, L., \& Bullough, A. (2010). Conceptualizing and Measuring Global Mindset@: Development of the Global Mindset Inventory. Glendale, AZ: Global Mindset Institute at Thunderbird School of Global Management.

Javidan, M., \& Walker, J. (2013). Developing your global mindset. Edina, MN: Beaver's Pond Press, Inc.

John, O. P., \& Robins, R. W. (1994). Accuracy and bias in self-perception: individual differences in self-enhancement and the role of narcissism. Journal of Personality and Social Psychology, 66, 206.

Johnson, R. B., \& Onwuegbuzie, A. J. (2004). Mixed methods research: A research paradigm whose time has come. Educational researcher, 33(7), 14-26.

Jokinen, T. (2005). Global leadership competencies: A review and discussion. Journal of European Industrial Training, 29, 199-216.

Kaiser, H. F. (1960). The application of electronic computers to factor analysis. Educational and psychological measurement, 20, 141-151.

Kaiser, H. F. (1970). A second generation little jiffy. Psychometrika, 35, 401-415.

Kegan, R. (1982). The evolving self: Problem and process in human development. Cambridge, MA: Harvard University Press.

Kegan, R. (1994). In over our heads: The mental demands of modern life. Cambridge, MA: Harvard University Press. 
Keeling, R. P. (2004). Learning reconsidered: A campus-wide focus on the student experience. Washington, DC: National Association of Student Personnel Administrators and American College Personnel Association.

Kets de Vries, M. F., Vrignaud, P., \& Florent-Treacy, E. (2004). The global leadership life inventory: Development and psychometric properties of a 360-degree feedback instrument. The International Journal of Human Resource Management, $15,475-492$.

Kitchener, K. S. (1986). The reflective judgment model: Characteristics, evidence, and measurement. In R. A. Mines \& K. S. Kitchener (Eds.), Adult cognitive development: Methods and models (pp. 76-91). New York, NY: Praeger.

Komives, S. R., Dugan, J. P., Owen, J. E., Slack, C., \& Wagner, W. (2011). The handbook for student leadership development. Hoboken, NJ: John Wiley \& Sons.

Komives, S. R., Lucas, N., \& McMahon, T. R. (2007). Exploring leadership: For college students who want to make a difference. San Francisco, CA: Jossey-Bass.

Kouzes, J.\& Posner, B. (2016). Leadership practices inventory. Retrieved from http://www.leadershipchallenge.com/professionals-section-lpi.aspx

Landorf, H., \& Doscher, S. (2015). Defining global learning at Florida International University. Retrieved July 30, 2016, from https://www.aacu.org/diversitydemocracy/2015/summer/landorf

Lane, H. W., Maznevski, M., Mendenhall, M. E., \& McNett, J. (Eds.). (2009). The Blackwell handbook of global management: A guide to managing complexity. Malden, MA: Blackwell Publishing Ltd.

Levy, O., Beechler, S., Taylor, S., \& Boyacigiller, N. A. (2007). What we talk about when we talk about 'Global Mindset': Managerial cognition in multinational corporations. Journal of International Business Studies, 38, 231-258.

Luft, J., \& Ingham, H. (1961). The Johari window. Human Relations Training News, 5, 6-7.

Matsumoto, D., \& Hwang, H. C. (2013). Assessing cross-cultural competence: A review of available tests. Journal of cross-cultural psychology. 44, 849-873.

McCauley, C. D., \& Van Velsor, E. (Eds.). (2004). The center for creative leadership handbook of leadership development (Vol. 29). Hoboken, NJ: John Wiley \& Sons.

McLeod, S. A. (2013). Kolb - Learning Styles. Retrieved from www.simplypsychology.org/learning-kolb.html 
Mendenhall, M. (2006). The elusive, yet critical challenge of developing global leaders. European Management Journal, 24(6), 422-429.

Mendenhall, M., \& Osland, J. S. (2002, June). Mapping the terrain of the global leadership construct. Symposium Presentation, Academy of International Business Annual Conference, San Juan, Puerto Rico.

Mendenhall, M. E., Osland, J., Bird, A., Oddou, G. R., Maznevski, M. L., Stevens, M., \& Stahl, G. K. (2013). Global leadership 2e: Research, practice, and development. New York, NY: Routledge.

Mendenhall, M. E., Reiche, B. S., Bird, A., \& Osland, J. S. (2012). Defining the "global" in global leadership. Journal of World Business, 47, 493-503.

Messick, S. (1989). Validity. In R. L. Linn (Ed.) Educational measurement (3rd ed., pp. 13-103). New York: Macmillan.

Messick, S. (1995). Validity of psychological assessment: Validation of inferences from persons' responses and performances as scientific inquiry into score meaning. American psychologist, 50(9), 741.

Messick, S. (1996). Validity of performance assessments. In G.W. Phillips (Ed.), Technical issues in large scale performance assessment (pp. 1-18). Washington, DC: National Center for Educational Statistics.

Meyers, L. S., Gamst, G., \& Guarino, A. J. (2006). Applied multivariate research: Design and interpretation. New York, NY: Sage.

Moore, L., Jenkins, D., Dietz, T., \& Feuerbaum, J. (1997). The use of the MBTI as a selfawareness tool in undergraduate education. J baccalaur soc work, 2, 53-70.

NACE. (2017). Career Readiness Defined. Retrieved from http://www.naceweb.org/career-readiness/competencies/career-readinessdefined/.

NAFSA. (2016). Internationalizing the curriculum. Retrieved from http://www.nafsa.org/Professional_Resources/Browse_by_Interest/Internationaliz ing_Higher_Education/Internationalizing_the_Curriculum/.

NASPA. (2016). Student affairs resources \& continued education | NASPA. Retrieved from https://www.naspa.org/about/student-affairs.

National Clearinghouse for Leadership Programs. (2016). Socially Responsible Leadership Scale-Revised Version Two. Retrieved from https://nclp.umd.edu/srls.aspx 
Newman, I., Lim, J., \& Pineda, F. (2013). Content validity using a mixed methods approach: Its application and development through the use of a table of specifications methodology. Journal of Mixed Methods Research, 7, 243-260.

Newman, I., Newman, D. and Newman, C. (2011). Writing research articles using mixed methods: Methodological considerations to help you get published. In T. S. Rocco and T. Hatcher (Eds). The handbook of scholarly writing and publishing (pp. 191208). San Francisco, CA: Jossey-Bass.

Osland, J.S. (2013). An overview of global leadership literature. Global leadership: Research, practice, and development, 40-79. New York, NY: Routledge.

Osland, J. S. \& Bird, A. (2006). "Global leaders as experts." In W. Mobley and E. Weldon (eds) Advances in Global Leadership, Volume 4 (pp. 123-142). Stamford, CT: JAI Press.

Osland, J. S., Bird, A., Mendenhall, M., \& Osland, A. (2006). Developing global leadership capabilities and global mindset: A review. Handbook of research in international human resource management, 197.

Pearman, R. R. (1999). Enhancing leadership effectiveness through psychological type: A development guide for using psychological type with executives, managers, supervisors, and team leaders. CAPT.

Perry, W. G. (1968). Patterns of development in thought and values of students in a liberal arts college: A validation of a scheme. Cambridge, MA: Bureau of Study, Counsel, Harvard University. (ERIC Document Reproduction Service No. ED024315)

Piaget, J. (1950). The psychology of intelligence. New York, NY: Routledge.

Reiche, B. S. \& Mendenhall, M. (2013). Looking to the future. Global leadership: Research, practice, and development, 260-268. New York, NY: Routledge.

Reio Jr, T. G., \& Shuck, B. (2015). Exploratory factor analysis: Implications for theory, research, and practice. Advances in Developing Human Resources, 17(1), 12-25.

Rhinesmith, S. H. (1993). A manager's guide to globalization: Six keys to success in a changing world. American Society for Training and Development. Alexandria, VA.

Rost, J.C. (1993). Leadership for the twenty-first century. Westport, CT: Praeger.

Sandeen, A., \& Barr, M. J. (2014). Critical issues for student affairs: Challenges and opportunities. Hoboken, NJ: John Wiley \& Sons. 
Seemiller, C. (2013). The student leadership competencies guidebook: Designing intentional leadership learning and development. Hoboken, NJ: John Wiley \& Sons.

Seemiller, C. (2016). Student leadership competencies. Retrieved from http://studentleadershipcompetencies.com.

Seemiller, C., \& Murray, T. (2013). The common language of leadership. Journal of Leadership Studies, 7, 33-45.

Seemiller, C., \& O'Keefe, S. (2016). Student leadership competencies program design handbook. Retrieved from www.studentleadershipcompetencies.com.

Shertzer, J. \& Doyle, M. (2006). "Student learning in leadership programs." In Komives, S. R., Dugan, J. P., Owen, J. E., Slack, C., \& Wagner, W. (2011). The handbook for student leadership development. Hoboken, NJ: John Wiley \& Sons.

Simon, M. K. (2010). Dissertations \& scholarly research: Recipes for success. Cottage Grove, OR: Dissertation Success.

Snyder, T. D., de Brey, C., \& Dillow, S. A. (2016). Digest of Education Statistics 2014, NCES 2016-006. National Center for Education Statistics.

Stevens, M., Bird, A., Mendenhall, M. E., \& Oddou, G. (2014). Measuring global leader intercultural competency: Development and validation of the Global Competencies Inventory (GCI). Advances in Global Leadership, 8, 115-154.

Suutari, V. (2002). Global leader development: An emerging research agenda. Career Development International, 7, 218-233.

Taneja, S., Sewell, S. S., \& Odom, R. Y. (2015). A culture of employee engagement: a strategic perspective for global managers. Journal of Business Strategy, 36, 46-56.

Taras, V., Rowney, J., \& Steel, P. (2009). Half a century of measuring culture: Review of approaches, challenges, and limitations based on the analysis of 121 instruments for quantifying culture. Journal of International Management, 15, 357-373.

Teddlie, C., \& Tashakkori, A. (2006). A general typology of research designs featuring mixed methods. Research in the Schools, 13, 12-28.

The Levin Institute. (2015). What is Globalization? Globalization101. Retrieved from http://www.globalization101.org/what-is-globalization/

The Myers \& Briggs Foundation. (2016). MBTI® Basics. Retrieved from http://www.myersbriggs.org/my-mbti-personality-type/mbti-basics/ 
Thorndike, R. M., \& Thorndike-Christ, T. (2010). Measurement and evaluation in psychology and education (8th ed). New York, NY: Pearson.

Tichy, N. M., Brimm, M., Charan, R., \& Takeuchi, H. (1992). “Leadership development as a lever for global transformation." In V. Pucik, N. Tichy, and C.K. Barnett (eds) Globalizing Management: Creating and leading the competitive organization. Hoboken, NJ: John Wiley \& Sons.

Tjan, A. (2012, July 19). How leaders become self-aware. Retrieved from https://hbr.org/2012/07/how-leaders-become-self-aware/

Tavakol, M., \& Dennick, R. (2011). Making sense of Cronbach's alpha. International journal of medical education, 2, 53.

Travers, C. J., Morisano, D., \& Locke, E. A. (2015). Self-reflection, growth goals, and academic outcomes: A qualitative study. British Journal of Educational Psychology, 85, 224-241.

True Colors Inc. (2016). About us - True Colors Intl. Retrieved July 30, 2016, from https://truecolorsintl.com/about-us/

Van Dyne, L., Ang, S., \& Koh, C. (2008). Development and validation of the CQS: The cultural intelligence scale. In S. Ang, \& L. Van Dyne, (Eds.). Handbook on cultural intelligence: Theory, measurement and applications (pp. 16-38). Armonk, NY: M.E. Sharpe.

Voronchenko, T., Klimenko, T., \& Kostina, I. (2015). Learning to live in a global world: Project-based learning in multicultural student groups as a pedagogy of tolerance strategy. Procedia-Social and Behavioral Sciences, 191, 1489-1495.

Wiley. (2016a). DiSC Profile - What is DiSC®? The DiSC personality test explained. Retrieved from https://www.discprofile.com/what-is-disc/overview/

Wiley. (2016b). Leadership Practices Inventory (LPI) assessments. Retrieved from http://www.leadershipchallenge.com/Leaders-Section-Assessments.aspx

Williams, B., Onsman, A., \& Brown, T. (2010). Exploratory factor analysis: A five-step guide for novices. Australasian Journal of Paramedicine, 8(3).

Willis, G. B. (2005). Cognitive interviewing: A tool for improving questionnaire design. Thousand Oaks, CA: Sage.

Yeung, A. K., \& Ready, D. A. (1995). Developing leadership capabilities of global corporations: A comparative study in eight nations. Human Resource Management, 34, 529-547. 
Yong, A. G., \& Pearce, S. (2013). A beginner's guide to factor analysis: Focusing on exploratory factor analysis. Tutorials in quantitative methods for psychology, 9(2), 79-94.

Yukl, G. (2006). Leadership in organizations ( $6^{\text {th }}$ ed.). Upper Saddle River, NJ: Pearson Prentice-Hall.

Zenger, J., Folkman, J., \& Evans, C. (2014). Global leadership development: how global organizations can successfully position leaders for global expansion. Retrieved from http://zengerfolkman.com/white-papers/.

Zenger, J. (2014). Are you starting too late? A head start on the path to extraordinary. Retrieved from http://zengerfolkman.com/white-papers/.

Zimmerman-Oster, K. and Burkhardt, J.C. (2000). Leadership in the making: Impact and insights from leadership development programs in US. colleges and universities. Battle Creek, MI: W.K. Kellogg Foundation. 
APPENDICES 
APPENDIX A

Sample Table of Specifications Letter 
Dear -----,

Thank you for your willingness to assist with my dissertation research. I consider you a subject matter expert and would like to get your feedback regarding the attached Table of Specifications. This is the first of four phases of research I will be conducting to demonstrate reliability and validity inferences for a Global Leadership Assessment.

\section{Brief Background}

For decades, institutions of higher education across the United States have offered cocurricular activities that help students develop leadership capabilities. In 2002, the American Council on Education released a report pointing out the shortcomings of the nation's international expertise and citizens' understanding of other cultures and global affairs. In 2007, after conducting an employer survey, the Association of American Colleges and Universities called for the need of global competence development for students. In 2017, the National Association of Colleges and Employers (NACE) identified eight competencies associated with career readiness, one of which centers on global competence development. This is Global/Intercultural Fluency, which NACE defines as "Value, respect, and learn from diverse cultures, races, ages, genders, sexual orientations, and religions. The individual demonstrates openness, inclusiveness, sensitivity, and the ability to interact respectfully with all people and understand individuals' differences.”

One of the greatest challenges of student affairs, the field that enhances student growth and development through co-curricular programs, is funding. Creating a new program that specifically focuses on global leadership competencies may not be feasible for most institutions. Instead, institutions of higher education have started to use the Student Leadership Competencies to help clearly identify learning outcomes of already existing co-curricular programs. These institutions publicize the Student Leadership Competencies associated with each of their programs, so students are able to identify the competencies they wish to enhance and choose co-curricular activities that develop those competencies. There is a need for a global leadership competency assessment that has terminology currently being used by leadership development programs in university settings such as that used in the Student Leadership Competencies. Universities provide students with the opportunity to develop their leadership skills, but the emerging area of concentration - global leadership - currently lacks appropriate resources and tools, such as a global leadership competency self-assessment instrument mapped within the Student Leadership Competencies.

\section{Table of Specifications Instructions}

In the attached excel document, you will find two tabs. In the first tab, labeled "NACE.SLC.GL Grid", I have organized the research from a review of the literature to help narrow down the Student Leadership Competencies (SLC) to be assessed by the Global Leadership assessment being created.

$>$ Column A has the NACE definition of Global/Intercultural fluency at the top and blank boxes below it. The NACE definition is what I am using as a starting point to define global leadership. As a result of your comments, I 
may use an alternate definition to express the concept of global leadership.

$>$ In the blank boxes I encourage you to answer these questions about two aspects for each SLC:

○ Does the sum of the descriptions from the global leadership literature adequately express the meaning of each SLC? Please comment.

$\bigcirc$ Is the SLC part of the NACE definition of Global/Intercultural fluency? Please comment.

$>$ Column B is each Student Leadership Competency (SLC) with its corresponding definition. The competencies that are highlighted in green are included in the questions on tab 2. Note: The competencies highlighted in green represent my initial decisions; these may change based on the feedback I receive from you. I encourage you to also review the non-highlighted competencies and to provide comments on the comparability between each SLC definition and its description based on the global leadership literature. $>$ Columns C - L are the Cultural Difference and Global Leadership (Education) Assessments. Here you will find the title of the instrument, the citation for the competency definitions, the competencies included in the assessment, and the definition of the competency (if available).

$>$ Columns $\mathrm{M}-\mathrm{Q}$ are Global Leadership Competency Frameworks and Models. These often provide a more detailed description of how the competencies are defined.

Again, I hope that you will review the analysis of the literature and provide comments in Column A. Row 4 provides an example of this. If there is not enough room for your comments, feel free to send a word document.

Tab 2 - "Behavioral Statements" includes potential items for the global leadership instrument. Column A includes the potential item; the rest of the columns include the competencies with their definitions. If you think the item can assess the corresponding competency, please indicate this in the box where the item row and competency column meet. Feel free to comment on the wording of the item especially if something is unclear or confusing. If you think an item can assess more than one competency, please be sure to mark all the competencies you believe it can assess.

I want to thank you again for your assistance in this process. I will try to incorporate your feedback to help refine both the competencies and the questions. I would appreciate if you could send me your feedback by May 26, 2017. I will follow up with you if I have questions regarding your feedback. Once my analysis is complete you will have an opportunity to make sure your feedback is included. If you have any questions, please do not hesitate to contact me.

Sincerely, Sabrena 


\section{APPENDIX B}

Visual Overview of Table of Specifications Tab 1 


\begin{tabular}{|c|c|c|c|c|c|c|c|c|c|c|c|c|c|c|c|c|}
\hline 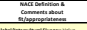 & & & & & & 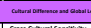 & (1) & & & & & & 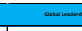 & 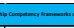 & netsone & \\
\hline $\begin{aligned}= \\
=\end{aligned}$ & 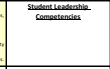 & 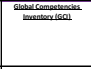 & 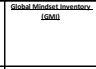 & 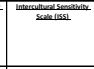 & 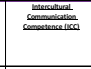 & 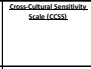 & 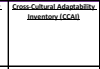 & 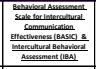 & 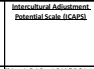 & t. & 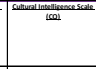 & 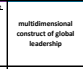 & 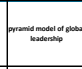 & 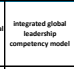 & stobes mininast & 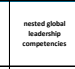 \\
\hline$=$ & $=$ & $=$ & $=$ & $\Rightarrow$ & 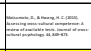 & 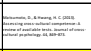 & $v_{y=1}=$ & $=$ & $\stackrel{=}{=}$ & $=$ & $=$ & $=$ & $=$ & $=$ & $=$ & $=$ \\
\hline$\frac{5}{5-m}$ & 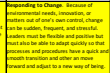 & 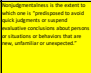 & & & & & $=$ & $=$ & & $=$ & & & $=$ & & & $-\infty$ \\
\hline & 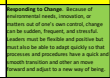 & 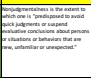 & & 4 & & & 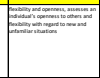 & 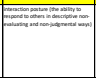 & & 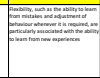 & & & 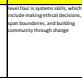 & & & 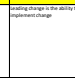 \\
\hline & man & 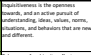 & $=$ & & & & & & 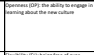 & 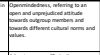 & 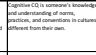 & 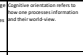 & Fits & 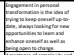 & & $=$ \\
\hline & $=$ & 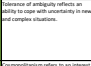 & 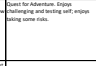 & & & & 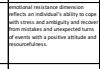 & 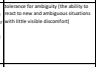 & 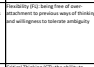 & 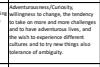 & & & & 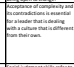 & 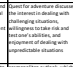 & $=$ \\
\hline & 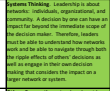 & 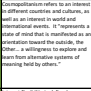 & & & & & & & 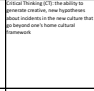 & & & 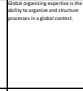 & $=$ & 作 & $=$ & $t=$ \\
\hline & $=$ & 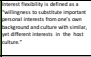 & 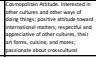 & & & 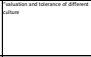 & 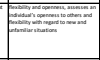 & 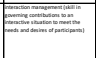 & & & & & & & 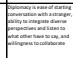 & wistons \\
\hline & $=$ & 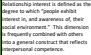 & 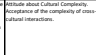 & & & mas & & 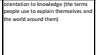 & & & 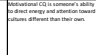 & & & $\begin{array}{ll}E=1 \\
z=\end{array}$ & 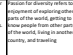 & \\
\hline & $=$ & 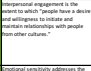 & 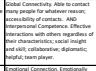 & & & & & 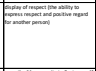 & & 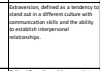 & & 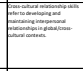 & 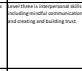 & $=$ & 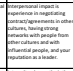 & $\begin{array}{l}5=4 \\
=\end{array}$ \\
\hline & $=$ & 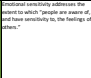 & 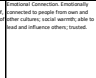 & & & & & ents & & 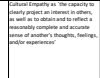 & & & & & 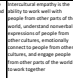 & \\
\hline & 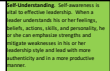 & $=$ & & & & & 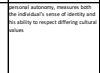 & & & & sints & & & $=$ & & 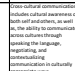 \\
\hline & $\begin{array}{l}=-1 \\
z=-1\end{array}$ & 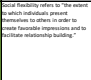 & 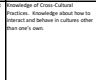 & & & 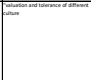 & 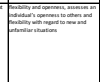 & & & & & & & & & 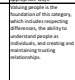 \\
\hline & $=$ & 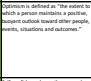 & 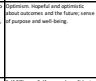 & & & & 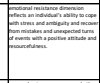 & & & & & & & 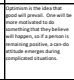 & & \\
\hline & $=$ & 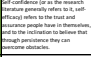 & 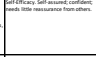 & & & & w & & & & & & & & $=$ & \\
\hline & 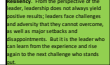 & 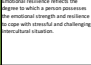 & 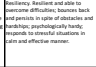 & & & & 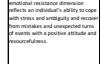 & & 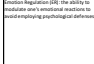 & 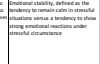 & & & & & & 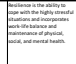 \\
\hline & 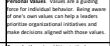 & 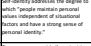 & & & & & 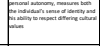 & & & & & & & & & \\
\hline & $=$ & 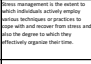 & sumbont & & & & & & & & & & & & & $m^{m+s}$ \\
\hline & 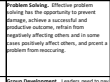 & & 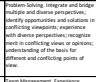 & & & & & wans & & & & & & 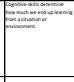 & 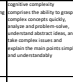 & \\
\hline & 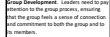 & & 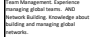 & & & & & $=0$ & & & & & & & & \\
\hline & 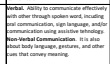 & & 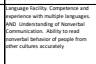 & & & & 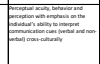 & & & & 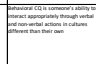 & & & & & \\
\hline & 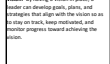 & & & & & & & & & & & westent & & & & $\begin{array}{l}n=1 \\
=1 \\
m=1\end{array}$ \\
\hline & 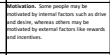 & 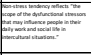 & & & & & & & & & & & & $=$ & & 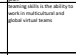 \\
\hline & 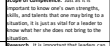 & & & & & & & & & & & & & 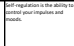 & & \\
\hline & 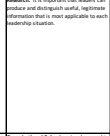 & & 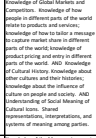 & & & & & & & & & $=$ minum & & 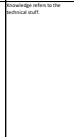 & 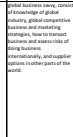 & 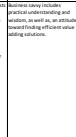 \\
\hline & 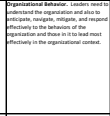 & & 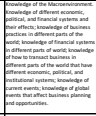 & & & & & & & & & & & & & 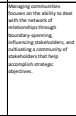 \\
\hline & 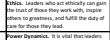 & & & & & & & & & & & & & & & sents \\
\hline & 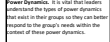 & & 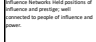 & & & & & & & & & & & & & \\
\hline & & & 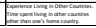 & & & & & & & & & & & & & \\
\hline
\end{tabular}




\section{APPENDIX B 1}

Global Leadership Competencies Literature used in the Table of Specifications Tab 1 


\section{Cultural Difference and Global Leadership - Education Assessments}

1. Global Competencies Inventory (GCI)

Stevens, M., Bird, A., Mendenhall, M. E., \& Oddou, G. (2014). Measuring global leader intercultural competency: Development and validation of the Global Competencies Inventory (GCI). Advances in global leadership, 8, 115-154.

- Nonjudgmentalness is the extent to which one is "predisposed to avoid quick judgments or suspend evaluative conclusions about persons or situations or behaviors that are new, unfamiliar or unexpected."

- Inquisitiveness is the openness towards, and an active pursuit of understanding, ideas, values, norms, situations, and behaviors that are new and different.

- Tolerance of ambiguity reflects an ability to cope with uncertainty in new and complex situations.

- Cosmopolitanism refers to an interest in different countries and cultures, as well as an interest in world and international events. It "represents a state of mind that is manifested as an orientation toward the outside, the Other... a willingness to explore and learn from alternative systems of meaning held by others."

- Interest flexibility is defined as a "willingness to substitute important personal interests from one's own background and culture with similar, yet different interests in the host culture."

- Relationship interest is defined as the degree to which "people exhibit interest in, and awareness of, their social environment." This dimension is frequently combined with others into a general construct that reflects interpersonal competence.

- Interpersonal engagement is the extent to which "people have a desire and willingness to initiate and maintain relationships with people from other cultures."

- Emotional sensitivity addresses the extent to which "people are aware of, and have sensitivity to, the feelings of others."

- Self-awareness is defined as the extent to which people possess awareness of themselves in their interactions with others.

- Social flexibility refers to "the extent to which individuals present themselves to others in order to create favorable impressions and to facilitate relationship building."

- Optimism is defined as "the extent to which a person maintains a positive, buoyant outlook toward other people, events, situations and outcomes."

- Self-confidence (or as the research literature generally refers to it, selfefficacy) refers to the trust and assurance people have in themselves, and to the inclination to believe that through persistence they can overcome obstacles.

- Emotional resilience reflects the degree to which a person possesses the emotional strength and resilience to cope with stressful and challenging intercultural situation. 
- Self-identity addresses the degree to which "people maintain personal values independent of situational factors and have a strong sense of personal identity."

- Stress management is the extent to which individuals actively employ various techniques or practices to cope with and recover from stress and also the degree to which they effectively organize their time.

- Non-stress tendency reflects "the scope of the dysfunctional stressors that may influence people in their daily work and social life in intercultural situations."

2. Global Mindset Inventory (GMI)

Javidan, M., \& Teagarden, M. B. (2011). Conceptualizing and measuring global mindset. In Advances in global leadership (pp. 13-39). Emerald Group Publishing Limited.

- Openness to New Ideas. Curious about ideas and people that are different; open-minded; enjoyment for learning about and experiencing new and different things.

- Quest for Adventure. Enjoys challenging and testing self; enjoys taking some risks.

- Cosmopolitan Attitude. Interested in other cultures and other ways of doing things; positive attitude toward international matters; respectful and appreciative of other cultures, their art forms, cuisine, and mores; passionate about crosscultural experiences.

- Attitude about Cultural Complexity. Acceptance of the complexity of crosscultural interactions.

- Global Connectivity. Able to contact many people for whatever reason; accessibility of contacts. AND Interpersonal Competence. Effective interactions with others regardless of their characteristics; social insight and skill; collaborative; diplomatic; helpful; team player.

- Emotional Connection. Emotionally connected to people from own and other cultures; social warmth; able to lead and influence others; trusted.

- Knowledge of Cross-Cultural Practices. Knowledge about how to interact and behave in cultures other than one's own.

- Optimism. Hopeful and optimistic about outcomes and the future; sense of purpose and well-being.

- Self-Efficacy. Self-assured; confident; needs little reassurance from others.

- Resiliency. Resilient and able to overcome difficulties; bounces back and persists in spite of obstacles and hardships; psychologically hardy; responds to stressful situations in calm and effective manner.

- Knowledge of Global Supply Chains. Understanding of the components of global supply chains.

- Problem-Solving. Integrate and bridge multiple and diverse perspectives; identify opportunities and solutions in conflicting viewpoints; experience with diverse perspectives; recognize merit in conflicting views or opinions; understanding of the basis for different and conflicting points of view. 
- Team Management. Experience managing global teams. AND Network Building. Knowledge about building and managing global networks.

- Language Facility. Competence and experience with multiple languages. AND Understanding of Nonverbal Communication. Ability to read nonverbal behavior of people from other cultures accurately

- Cognitive Ability. Ability to understand complex global issues.

- Knowledge of Global Markets and Competitors. Knowledge of how people in different parts of the world relate to products and services; knowledge of how to tailor a message to capture market share in different parts of the world; knowledge of product pricing and entry in different parts of the world. AND Knowledge of Cultural History. Knowledge about other cultures and their histories; knowledge about the influence of culture on people and society. AND Understanding of Social Meaning of Cultural Icons. Shared representations, interpretations, and systems of meaning among parties.

- Knowledge of the Macroenvironment. Knowledge of different economic, political, and financial systems and their effects; knowledge of business practices in different parts of the world; knowledge of financial systems in different parts of world; knowledge of how to transact business in different parts of the world that have different economic, political, and institutional systems; knowledge of current events; knowledge of global events that affect business planning and opportunities.

- Influence Networks Held positions of influence and prestige; well connected to people of influence and power.

- Experience Living in Other Countries. Time spent living in other countries other than one's home country.

3. Intercultural Sensitivity Scale (ISS)

Matsumoto, D., \& Hwang, H. C. (2013). Assessing cross-cultural competence: A review of available tests. Journal of cross-cultural psychology. 44, 849-873.

- Suspending judgment

- Open-mindedness

- Interaction involvement
- Empathy

- Self-monitoring

- Self-esteem

4. Intercultural Communication Competence (ICC)

Matsumoto, D., \& Hwang, H. C. (2013). Assessing cross-cultural competence: A review of available tests. Journal of cross-cultural psychology. 44, 849-873.

- Global attitude

- Intercultural experience and training
- Ability to listen well in conversation

- Empathy

- Motivation

5. Cross-Cultural Sensitivity Scale (CCSS)

Matsumoto, D., \& Hwang, H. C. (2013). Assessing cross-cultural competence: A review of available tests. Journal of cross-cultural psychology. 44, 849-873.

- "Valuation and tolerance of different culture" 
6. Cross-Cultural Adaptability Inventory (CCAI)

Sinicrope, C., Norris, J., \& Watanabe, Y. (2007). Understanding and assessing intercultural competence: A summary of theory, research, and practice

(Technical report for the Foreign Language Program Evaluation Project).

University of Hawai'I Second Langauge Studies Paper 26 (1).

- Flexibility and openness, assesses an individual's openness to others and flexibility with regard to new and unfamiliar situations

- Emotional resistance dimension reflects an individual's ability to cope with stress and ambiguity and recover from mistakes and unexpected turns of events with a positive attitude and resourcefulness.

- Personal autonomy, measures both the individual's sense of identity and his ability to respect differing cultural values

- Perceptual acuity, behavior and perception with emphasis on the individual's ability to interpret communication cues (verbal and non-verbal) crossculturally

7. Behavioral Assessment Scale for Intercultural Communication Effectiveness (BASIC) \& Intercultural Behavioral Assessment (IBA)

Koester, J., \& Olebe, M. (1988). The behavioral assessment scale for intercultural communication effectiveness. International Journal of Intercultural Relations, 12(3), 233-246.

- Interaction posture (the ability to respond to others in descriptive nonevaluating and non-judgmental ways)

- Tolerance for ambiguity (the ability to react to new and ambiguous situations with little visible discomfort)

- Interaction management (skill in governing contributions to an interactive situation to meet the needs and desires of participants)

- Orientation to knowledge (the terms people use to explain themselves and the world around them)

- Display of respect (the ability to express respect and positive regard for another person)

- Empathy (the capacity to "put oneself in another's shoes" or to behave as if one could)

- Task Role Behavior: Behaviors that involve the initiation of ideas related to group problem-solving activities

- Rational Role Behavior: Behaviors Associated with interpersonal harmony and mediation

8. Intercultural Adjustment Potential Scale (ICAPS)

Matsumoto, D., LeRoux, J., Ratzlaff, C., Tatani, H., Uchida, H., Kim, C., \& Araki, S. (2001). Development and validation of a measure of intercultural adjustment potential in Japanese sojourners: The Intercultural Adjustment Potential Scale (ICAPS). International Journal of Intercultural Relations, 25(5), 483-510. 
- Openness (OP): the ability to engage in learning about the new culture

- Flexibility (FL): being free of over-attachment to previous ways of thinking and willingness to tolerate ambiguity

- Critical Thinking (CT): the ability to generate creative, new hypotheses about incidents in the new culture that go beyond one's home cultural framework

- Emotion Regulation (ER): the ability to modulate one's emotional reactions to avoid employing psychological defenses

9. Multicultural Personality Inventory (MPQ)

Van Der Zee, K. I., \& Van Oudenhoven, J. P. (2000). The Multicultural

Personality Questionnaire: A multidimensional instrument of multicultural

effectiveness. European journal of personality, 14(4), 291-309.

- Flexibility, such as the ability to learn from mistakes and adjustment of behaviour whenever it is required, are particularly associated with the ability to learn from new experiences

- Openmindedness, referring to an open and unprejudiced attitude towards outgroup members and towards different cultural norms and values.

- Adventurousness/Curiosity, willingness to change, the tendency to take on more and more challenges and to have adventurous lives, and the wish to experience different cultures and to try new things also tolerance of ambiguity.

- Extraversion, defined as a tendency to stand out in a different culture with communication skills and the ability to establish interpersonal relationships.

- Cultural Empathy as 'the capacity to clearly project an interest in others, as well as to obtain and to reflect a reasonably complete and accurate sense of another's thoughts, feelings, and/or experiences'

- Emotional stability, defined as the tendency to remain calm in stressful situations versus a tendency to show strong emotional reactions under stressful circumstance

10. Cultural Intelligence Scale (CQ)

Van Dyne, L., Ang, S., \& Koh, C. (2008). Development and validation of the CQS. Handbook of Cultural Intelligence, 16-40.

- Cognitive CQ is someone's knowledge and understanding of norms, practices, and conventions in cultures different from their own.

- Motivational CQ is someone's ability to direct energy and attention toward cultures different than their own.

- Metacognitive CQ is someone's awareness and conscientiousness while interacting with cultures different than their own.

- Behavioral CQ is someone's ability to interact appropriately through verbal and non-verbal actions in cultures different than their own 


\section{Global Leadership Competency Frameworks and Models}

11. Multidimensional Construct of Global Leadership Mendenhall, M., \& Osland, J.S. (2002, June). Mapping the terrain of the global leadership construct. Symposium Presentation, Academy of International Business Annual Conference, San Juan, Puerto Rico.

- Cognitive orientation is how one processes information and their world-view.

- Global organizing expertise is the ability to organize and structure processes in a global context.

- Cross-cultural relationship skills refer to developing and maintaining interpersonal relationships in global/cross-cultural contexts.

- Traits and values are personality traits and habits.

- Visioning is knowing where the company should be heading and knowing how to get others to help achieve that vision

- Global business expertise is knowledge about the global business practices.

\section{Integrated Global Leadership Competency Model}

Jokinen, T. (2005). Global leadership competencies: A review and discussion. Journal of European Industrial Training, 29, 199-216.

- Engagement in personal transformation is the idea of trying to keep oneself up-to-date, always looking for new opportunities to learn and enhance oneself as well as being open to change.

- Acceptance of complexity and its contradictions is essential for a leader that is dealing with a culture that is different from their own.

- Social judgment skills refer to the ability to look beyond the situation to the bigger picture. It's a systems approach that allows a person to switch viewpoints and understand interdependence.

- Inquisitiveness, on the other hand, is curiosity and the desire to find new information about old topics.

- Social skills are more informal person-to-person interactions.

- Empathy is having a genuine concern for others' needs and perceptions.

- To be self-aware indicates that a person is knowledgeable of their strengths, weaknesses, desires, motivators, and typical reactions.

- Optimism is the idea that good will prevail. One will be more motivated to do something that they believe will happen, so if a person is remaining positive, a can-do attitude emerges during complicated situations.

- Cognitive skills determine how much we end up learning from a situation or environment.

- Motivation to work in an international environment is important or a person will lose motivation quickly if they never wanted to work with an international population in the first place.

- Self-regulation is the ability to control your impulses and moods.

- Knowledge refers to the technical stuff.

- Network management skills refer to formal relationships created through organizations 


\section{Global Mindset}

Javidan, M., \& Walker, J. (2013). Developing your global mindset. Edina, MN:

Beaver's Pond Press, Inc.

- Quest for adventure discusses the interest in dealing with challenging situations, willingness to take risk and test one's abilities, and enjoyment of dealing with unpredictable situations

- Cosmopolitan outlook, which includes knowledge of cultures in different parts of the world, geography, history, and important persons of several countries, economic and political issues, concerns, and hot topics of major regions of the world, and important world events

- Diplomacy is ease of starting conversation with a stranger, ability to integrate diverse perspectives and listen to what other have to say, and willingness to collaborate

- Passion for diversity refers to enjoyment of exploring other parts of the world, getting to know people from other parts of the world, living in another country, and traveling

- Interpersonal impact is experience in negotiating contract/agreements in other cultures, having strong networks with people from other cultures and with influential people, and your reputation as a leader.

- Intercultural empathy is the ability to work well with people from other parts of the world, understand nonverbal expressions of people from other cultures, emotionally connect to people from other cultures, and engage people from other parts of the world to work together

- Self-assurance is being energetic, self-confident, comfortable in uncomfortable situations, and witty in touch situations

- Cognitive complexity comprises the ability to grasp complex concepts quickly, analyze and problem-solve, understand abstract ideas, and take complex issues and explain the main points simply and understandably

- Global business savvy, consists of knowledge of global industry, global competitive business and marketing strategies, how to transact business and assess risks of doing business internationally, and supplier options in other parts of the world.

\section{Nested Global Leadership Competencies}

Bird, A. (2013). Mapping the content domain of global leadership competencies. Global leadership: Research, practice, and development, 80-96. New York, NY: Routledge.

- Leading change is the ability to implement change

- Inquisitiveness is an innate curiosity, being open-minded, having humility, and life-long learning.

- Flexibility is the willingness to tolerate ambiguity and adapt to various situations.

- Global mindset includes having cognitive complexity and cosmopolitanism 
- Empowering others is energizing individuals by increasing their self-efficacy.

- Interpersonal skills are broken into the broad definitions of emotional intelligence and relationship management skills.

- Cross-cultural communication includes cultural awareness of both self and others, as well as, the ability to communicate across cultures through speaking the language, negotiating, and contextualizing communication in culturally appropriate ways.

- Valuing people is the foundation of this category, which includes respecting differences, the ability to understand people as individuals, and creating and maintaining trusting relationships.

- Resilience is the ability to cope with the highly stressful situations and incorporates work-life balance and maintenance of physical, social, and mental health.

- Organizational savvy is the ability to design global organizational structures and processes.

- Vision and strategic thinking encompasses the ability to comprehend and strategically think about the complexity of the environment, activities related to developing and articulating a global vision, and the ability to develop and implement a global strategic plan.

- Teaming skills is the ability to work in multicultural and global virtual teams

- Business savvy includes practical understanding and wisdom, as well as, an attitude toward finding efficient value adding solutions.

- Managing communities focuses on the ability to deal with the network of relationships through boundary-spanning, influencing stakeholders, and cultivating a community of stakeholders that help accomplish strategic objectives.

- Character is a combination of integrity, maturity, and conscientiousness. 
APPENDIX B2

Seemiller's Student Leadership Competencies used in the Table of Specifications Tab 1 
$\underline{\text { Student Leadership Competencies }}$

Seemiller, C. (2013). The Student Leadership Competencies Guidebook: Designing

Intentional Leadership Learning and Development. John Wiley \& Sons.

1. Responding to Change. Because of environmental needs, innovation, or matters out of one's own control, change can be sudden, frequent, and stressful. Leaders must be flexible and positive but must also be able to adapt quickly so that processes and procedures have a quick and smooth transition and other an move forward and adjust to a new way of being.

2. Self-Development. Whether it is learning new technology or how to become a better public speaker, leaders are always learning.

3. Responding to Ambiguity. Because leaders cannot truly control all circumstances and will never know all the answers, they must be able to respond to uncertainty and the unknown.

4. Systems Thinking. Leadership is about networks: individuals, organizational, and community. A decision by one can have an impact far beyond the immediate scope of the decision maker. Therefore, leaders must be able to understand how networks work and be able to navigate through both the ripple effects of others' decisions as well as engage in their own decision making that considers the impact on a larger network or system.

5. Others Perspectives. Leaders do not have all the answers. Thus, it is essential that they are able to truly consider other opinions, experiences, and outlooks to help them develop better solutions and approaches when dealing with leadership situations.

6. Diversity. Leadership is inherently an interpersonal process, and in many situations, leaders will find themselves in the position of working with individuals who have different backgrounds, beliefs, and/or experiences than they do.

7. Productive Relationships. Leadership requires that a leader has meaningful connections with others; simply interacting with people does not constitute a relationship.

8. Empathy. Not only can demonstrating empathy with others build relationships and a sense of trust, it can also help a leader understand another point of view or other set of circumstances to effectively inform the leader's decisions and actions.

9. Self-Understanding. Self-awareness is vital to effective leadership. When a leader understands his or her feelings, beliefs, actions, skills, and personality, he or she can emphasize strengths and mitigate weaknesses in his or her leadership style and lead with more authenticity and in a more productive manner.

10. Others' Circumstances. It is critical for leaders to seek to understand the situations and/or conditions of other people. This understanding not only helps inform the leader's decisions but also helps the leader be conscious of what others have experienced or are experiencing so as to engage in inclusive behaviors and connect with others with a sense of care. 
11. Positive Attitude. Life is full of unexpected challenges, changes, and actions by others that can be discouraging or defeating. Attitude plays an important role in how one deals with these circumstances. A leader with a positive attitude can foster a sense of optimism, hope, inspiration, and enthusiasm even if the circumstances are bad.

12. Confidence. People look to leaders to give them inspiration and assurance. That is why demonstrating confidence is so essential. Followers must be able to believe in their leaders; in turn, leaders must look like they believe in themselves.

13. Resiliency. From the perspective of the leader, leadership does not always yield positive results; leaders face challenges and adversity that they cannot overcome, as well as major setbacks and disappointments. But it is the leader who can learn from the experience and rise again to the next challenge who stands out.

14. Personal Values. Values are a guiding force for individual behavior. Being aware of one's own values can help a leaders prioritize organizational initiatives and make decisions aligned with those values.

15. Organization. In addition to the human relations aspect, leadership is often about managing several moving pats such as information, resources, and materials. Leaders need to create systems and structures to most effectively manage, monitor, and utilize these moving parts.

16. Problem Solving. Effective problem solving has the opportunity to prevent damage, achieve a successful and productive outcome, refrain from negatively affecting others and in some cases positively affect others, and percent a problem from reoccurring.

17. Group Development. Leaders need to pay attention to the group process, ensuring that the group feels a sense of connection and commitment to both the group and to its members.

18. Verbal. Ability to communicate effectively with other through spoken word, including oral communication, sign language, and/or communication using assistive technology. Non-Verbal Communication. It is also about body language, gestures, and other cues that convey meaning.

19. Vision. By having a vision in place, a leader can develop goals, plans, and strategies that align with the vision so as to stay on track, keep motivated, and monitor progress toward achieving the vision.

20. Motivation. Some people may be motivated by internal factors such as drive and desire, whereas others may be motivated by external factors like rewards and incentives.

21. Scope of Competence. Just as it is important to know one's own strengths, skills, and talents that one may bring to a situation, it is just as vital for a leader to know what her she does not bring to the situation.

22. Research. It is important that leaders can produce and distinguish useful, legitimate information that is most applicable to each leadership situation.

23. Organizational Behavior. Leaders need to understand the organization and also to anticipate, navigate, mitigate, and respond effectively to the behaviors of the organization and those in it to lead most effectively in the organizational context. 
24. Ethics. Leaders who act ethically can gain the trust of those they work with, inspire others to greatness, and fulfill the duty of care for those they lead.

25. Power Dynamics. It is vital that leaders understand the types of power dynamics that exist in their groups so they can better respond to the group's needs within the context of these power dynamics. 
APPENDIX C

Visual Overview of Table of Specifications Tab 2 


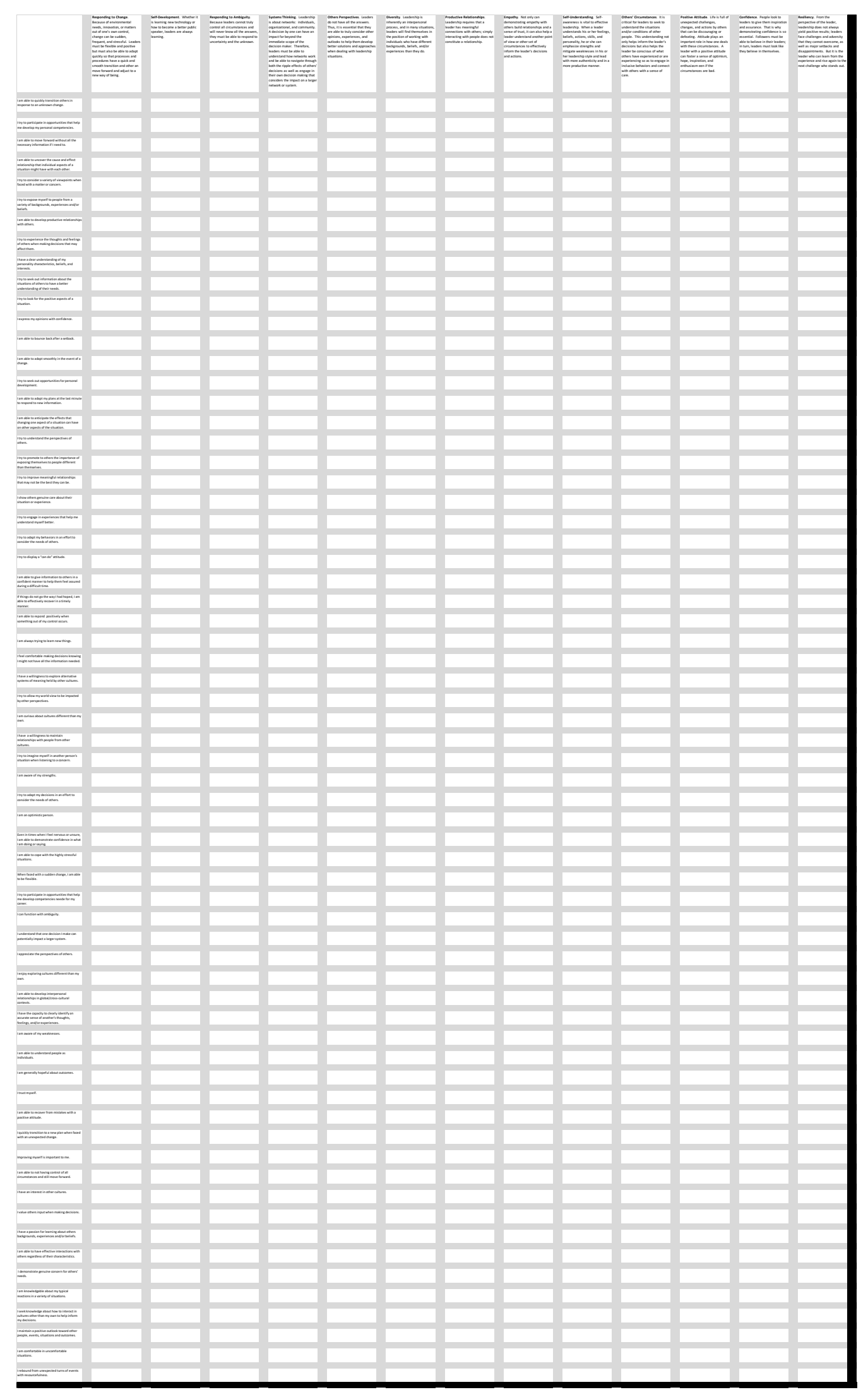




\section{APPENDIX C1}

Seemiller's Student Leadership Competencies included in the First Version of Tab 2 
$\underline{\text { Student Leadership Competencies }}$

Seemiller, C. (2013). The Student Leadership Competencies Guidebook: Designing

Intentional Leadership Learning and Development. John Wiley \& Sons.

1. Responding to Change. Because of environmental needs, innovation, or matters out of one's own control, change can be sudden, frequent, and stressful. Leaders must be flexible and positive but must also be able to adapt quickly so that processes and procedures have a quick and smooth transition and other an move forward and adjust to a new way of being.

2. Self-Development. Whether it is learning new technology or how to become a better public speaker, leaders are always learning.

3. Responding to Ambiguity. Because leaders cannot truly control all circumstances and will never know all the answers, they must be able to respond to uncertainty and the unknown.

4. Systems Thinking. Leadership is about networks: individuals, organizational, and community. A decision by one can have an impact far beyond the immediate scope of the decision maker. Therefore, leaders must be able to understand how networks work and be able to navigate through both the ripple effects of others' decisions as well as engage in their own decision making that considers the impact on a larger network or system.

5. Others Perspectives. Leaders do not have all the answers. Thus, it is essential that they are able to truly consider other opinions, experiences, and outlooks to help them develop better solutions and approaches when dealing with leadership situations.

6. Diversity. Leadership is inherently an interpersonal process, and in many situations, leaders will find themselves in the position of working with individuals who have different backgrounds, beliefs, and/or experiences than they do.

7. Productive Relationships. Leadership requires that a leader has meaningful connections with others; simply interacting with people does not constitute a relationship.

8. Empathy. Not only can demonstrating empathy with others build relationships and a sense of trust, it can also help a leader understand another point of view or other set of circumstances to effectively inform the leader's decisions and actions.

9. Self-Understanding. Self-awareness is vital to effective leadership. When a leader understands his or her feelings, beliefs, actions, skills, and personality, he or she can emphasize strengths and mitigate weaknesses in his or her leadership style and lead with more authenticity and in a more productive manner.

10. Others' Circumstances. It is critical for leaders to seek to understand the situations and/or conditions of other people. This understanding not only helps inform the leader's decisions but also helps the leader be conscious of what others have experienced or are experiencing so as to engage in inclusive behaviors and connect with others with a sense of care. 
11. Positive Attitude. Life is full of unexpected challenges, changes, and actions by others that can be discouraging or defeating. Attitude plays an important role in how one deals with these circumstances. A leader with a positive attitude can foster a sense of optimism, hope, inspiration, and enthusiasm even if the circumstances are bad.

12. Confidence. People look to leaders to give them inspiration and assurance. That is why demonstrating confidence is so essential. Followers must be able to believe in their leaders; in turn, leaders must look like they believe in themselves.

13. Resiliency. From the perspective of the leader, leadership does not always yield positive results; leaders face challenges and adversity that they cannot overcome, as well as major setbacks and disappointments. But it is the leader who can learn from the experience and rise again to the next challenge who stands out. 


\section{APPENDIX C2}

Questions included in the First Version of Tab 2 
1. I am able to quickly transition others in response to an unknown change.

2. I try to participate in opportunities that help me develop my personal competencies.

3. I am able to move forward without all the necessary information if I need to.

4. I am able to uncover the cause and effect relationship that individual aspects of a situation might have with each other.

5. I try to consider a variety of viewpoints when faced with a matter or concern.

6. I try to expose myself to people from a variety of backgrounds, experiences and/or beliefs.

7. I am able to develop productive relationships with others.

8. I try to experience the thoughts and feelings of others when making decisions that may affect them.

9. I have a clear understanding of my personality characteristics, beliefs, and interests.

10. I try to seek out information about the situations of others to have a better understanding of their needs.

11. I try to look for the positive aspects of a situation.

12. I express my opinions with confidence.

13. I am able to bounce back after a setback.

14. I am able to adapt smoothly in the event of a change.

15. I try to seek out opportunities for personal development.

16. I am able to adapt my plans at the last minute to respond to new information.

17. I am able to anticipate the effects that changing one aspect of a situation can have on other aspects of the situation.

18. I try to understand the perspectives of others.

19. I try to promote to others the importance of exposing themselves to people different than themselves.

20. I try to improve meaningful relationships that may not be the best they can be.

21 . I show others genuine care about their situation or experience.

22. I try to engage in experiences that help me understand myself better.

23 . I try to adapt my behaviors in an effort to consider the needs of others.

24. I try to display a "can do" attitude.

25. I am able to give information to others in a confident manner to help them feel assured during a difficult time.

26. If things do not go the way I had hoped, I am able to effectively recover in a timely manner.

27. I am able to respond positively when something out of my control occurs.

28. I am always trying to learn new things.

29. I feel comfortable making decisions knowing I might not have all the information needed.

30. I have a willingness to explore alternative systems of meaning held by other cultures.

31. I try to allow my world view to be impacted by other perspectives.

32. I am curious about cultures different than my own.

33. I have a willingness to maintain relationships with people from other cultures. 
34. I try to imagine myself in another person's situation when listening to a concern.

35. I am aware of my strengths.

36. I try to adapt my decisions in an effort to consider the needs of others.

37. I am an optimistic person.

38. Even in times when I feel nervous or unsure, I am able to demonstrate confidence in what I am doing or saying.

39. I am able to cope with the highly stressful situations.

40. When faced with a sudden change, I am able to be flexible.

41. I try to participate in opportunities that help me develop competencies need for my career.

42. I can function with ambiguity.

43. I understand that one decision I make can potentially impact a larger system.

44. I appreciate the perspectives of others.

45. I enjoy exploring cultures different than my own.

46. I am able to develop interpersonal relationships in global/cross-cultural contexts.

47. I have the capacity to clearly identify an accurate sense of another's thoughts, feelings, and/or experiences.

48. I am aware of my weaknesses.

49. I am able to understand people as individuals.

50. I am generally hopeful about outcomes.

51. I trust myself.

52. I am able to recover from mistakes with a positive attitude.

53. I quickly transition to a new plan when faced with an unexpected change.

54. Improving myself is important to me.

55. I am able to not having control of all circumstances and still move forward.

56. I have an interest in other cultures.

57. I value others input when making decisions.

58. I have a passion for learning about others backgrounds, experiences and/or beliefs.

59. I am able to have effective interactions with others regardless of their characteristics.

60. I demonstrate genuine concern for others' needs.

61. I am knowledgeable about my typical reactions in a variety of situations.

62. I seek knowledge about how to interact in cultures other than my own to help inform my decisions.

63. I maintain a positive outlook toward other people, events, situations and outcomes.

64. I am comfortable in uncomfortable situations.

65. I rebound from unexpected turns of events with resourcefulness. 


\section{APPENDIX D}

Sample of Tab 2 Update Email to Experts 


\section{Greetings ---,}

Thank you again for being willing to assist me with my dissertation. I have already received some good critical feedback from this process, so I will be changing most of tab 2. This means that you only need to look at and comment about tab 1 by May 31. You will receive a different tab 2 sometime after that. This new process should actually save you time in the long run. If you have any questions, please let me know!

Sincerely,

Sabrena 


\section{APPENDIX E}

Sample New Tab 2 Letter 


\section{Dear ---,}

Thank you again for your willingness to assist with my dissertation research. In particular, I would like to thank you for serving as a subject matter expert and giving me your opinion on the fit of the Student Leadership Competencies, Global Leadership Research, and NACE definition back in May. After several revisions throughout the summer, I am requesting that you again provide me with your opinion, but this time on the questions that have the potential to be on the global leadership assessment I am creating. The analysis of your feedback with the other subject matter experts' feedback will complete the first of four phases of research I will be conducting to demonstrate reliability and validity inferences for a global leadership assessment.

\section{Instructions}

In the attached excel document, you will find the potential items (questions) for the global leadership instrument. Column A includes the potential items; the rest of the columns include the competencies with their definitions. If you think the item can assess the corresponding competency, please indicate this in the box where the item row and competency column meet with the word "yes." If you do not think it assesses that competency, either write "no" or leave it blank. If you think an item can assess more than one competency, please mark all the competencies you believe it can assess. Also, feel free to comment on the wording of the item especially if something is unclear or confusing. Row 3 in the excel provides an example of this.

I want to thank you again for your assistance in this process. Your feedback is essential in helping me validate my instrument. I would appreciate if you could send me your feedback by Sept. 29, 2017. If you have any questions, please do not hesitate to contact me.

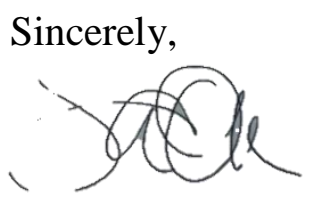




\section{APPENDIX F}

Visual Overview of Final Version of Tab 2 


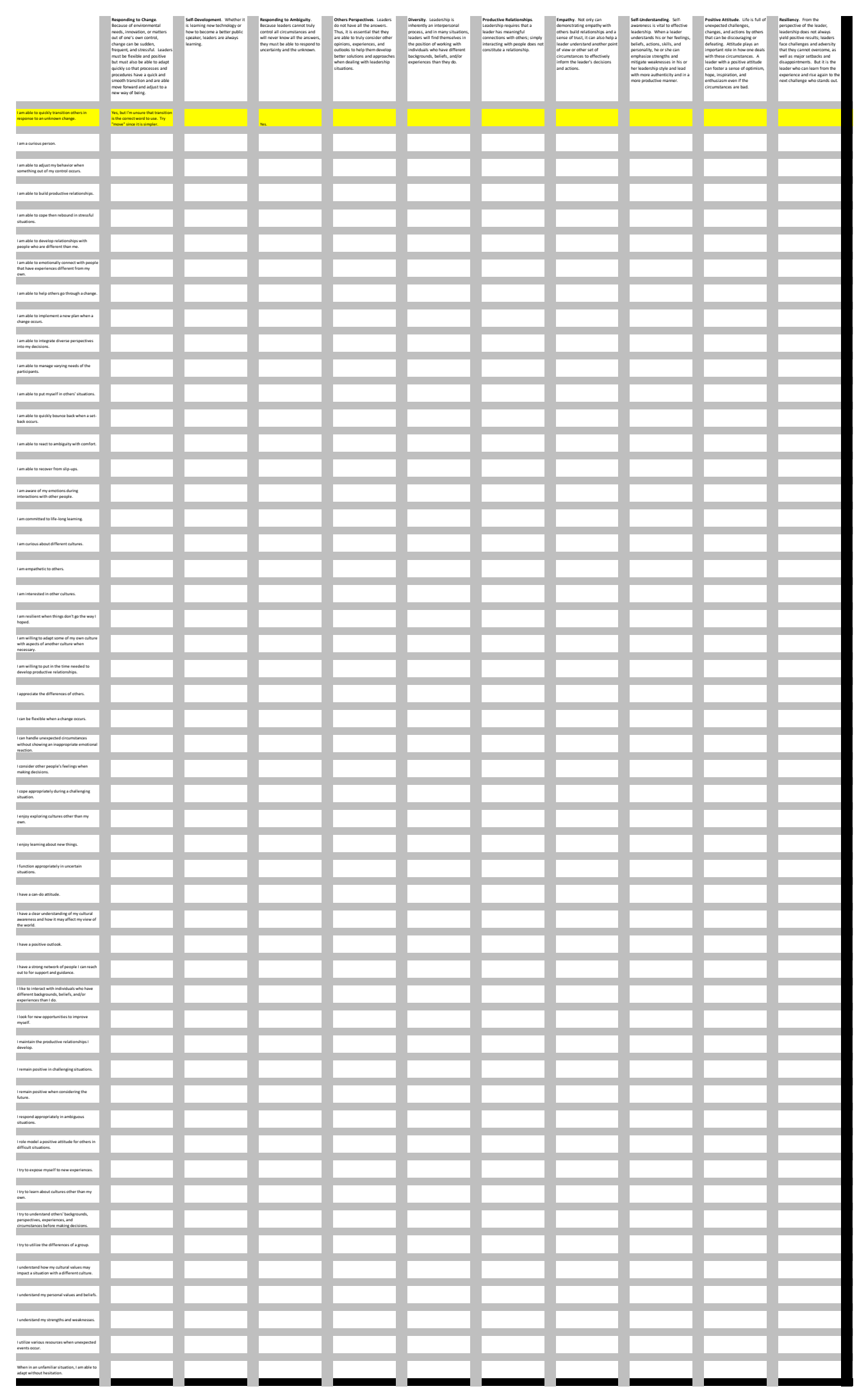




\section{APPENDIX F1}

Seemiller's Student Leadership Competencies included in the Final Version of Tab 2 
1. Responding to Change. Because of environmental needs, innovation, or matters out of one's own control, change can be sudden, frequent, and stressful. Leaders must be flexible and positive but must also be able to adapt quickly so that processes and procedures have a quick and smooth transition and are able move forward and adjust to a new way of being.

2. Self-Development. Whether it is learning new technology or how to become a better public speaker, leaders are always learning.

3. Responding to Ambiguity. Because leaders cannot truly control all circumstances and will never know all the answers, they must be able to respond to uncertainty and the unknown.

4. Others Perspectives. Leaders do not have all the answers. Thus, it is essential that they are able to truly consider other opinions, experiences, and outlooks to help them develop better solutions and approaches when dealing with leadership situations.

5. Diversity. Leadership is inherently an interpersonal process, and in many situations, leaders will find themselves in the position of working with individuals who have different backgrounds, beliefs, and/or experiences than they do.

6. Productive Relationships. Leadership requires that a leader has meaningful connections with others; simply interacting with people does not constitute a relationship.

7. Empathy. Not only can demonstrating empathy with others build relationships and a sense of trust, it can also help a leader understand another point of view or other set of circumstances to effectively inform the leader's decisions and actions.

8. Self-Understanding. Self-awareness is vital to effective leadership. When a leader understands his or her feelings, beliefs, actions, skills, and personality, he or she can emphasize strengths and mitigate weaknesses in his or her leadership style and lead with more authenticity and in a more productive manner.

9. Positive Attitude. Life is full of unexpected challenges, changes, and actions by others that can be discouraging or defeating. Attitude plays an important role in how one deals with these circumstances. A leader with a positive attitude can foster a sense of optimism, hope, inspiration, and enthusiasm even if the circumstances are bad.

10. Resiliency. From the perspective of the leader, leadership does not always yield positive results; leaders face challenges and adversity that they cannot overcome, as well as major setbacks and disappointments. But it is the leader who can learn from the experience and rise again to the next challenge who stands out. 


\section{APPENDIX F2}

Questions include in the Final Version of Tab 2 
1. I am a curious person.

2. I am able to adjust my behavior when something out of my control occurs.

3. I am able to build productive relationships.

4. I am able to cope then rebound in stressful situations.

5. I am able to develop relationships with people who are different than me.

6. I am able to emotionally connect with people that have experiences different from my own.

7. I am able to help others go through a change.

8. I am able to implement a new plan when a change occurs.

9. I am able to integrate diverse perspectives into my decisions.

10. I am able to manage varying needs of the participants.

11. I am able to put myself in others' situations.

12. I am able to quickly bounce back when a set-back occurs.

13. I am able to react to ambiguity with comfort.

14. I am able to recover from slip-ups.

15. I am aware of my emotions during interactions with other people.

16. I am committed to life-long learning.

17. I am curious about different cultures.

18. I am empathetic to others.

19. I am interested in other cultures.

20. I am resilient when things don't go the way I hoped.

21. I am willing to adapt some of my own culture with aspects of another culture when necessary.

22. I am willing to put in the time needed to develop productive relationships.

23. I appreciate the differences of others.

24. I can be flexible when a change occurs.

25. I can handle unexpected circumstances without showing an inappropriate emotional reaction.

26. I consider other people's feelings when making decisions.

27. I cope appropriately during a challenging situation.

28. I enjoy exploring cultures other than my own.

29. I enjoy learning about new things.

30. I function appropriately in uncertain situations.

31. I have a can-do attitude.

32. I have a clear understanding of my cultural awareness and how it may affect my view of the world.

33. I have a positive outlook.

34. I have a strong network of people I can reach out to for support and guidance.

35. I like to interact with individuals who have different backgrounds, beliefs, and/or experiences than I do.

36. I look for new opportunities to improve myself.

37. I maintain the productive relationships I develop.

38. I remain positive in challenging situations.

39. I remain positive when considering the future.

40. I respond appropriately in ambiguous situations. 
41. I role model a positive attitude for others in difficult situations.

42. I try to expose myself to new experiences.

43. I try to learn about cultures other than my own.

44. I try to understand others' backgrounds, perspectives, experiences, and circumstances before making decisions.

45. I try to utilize the differences of a group.

46. I understand how my cultural values may impact a situation with a different culture.

47. I understand my personal values and beliefs.

48. I understand my strengths and weaknesses.

49. I utilize various resources when unexpected events occur.

50. When in an unfamiliar situation, I am able to adapt without hesitation. 


\section{APPENDIX G}

Email sent to Experts Requesting Consultation 
Hello Dr. ---,

Hope you are doing well! After calculating the results/comments from 13 subject matter experts regarding potential questions for my global leadership instrument, I ended up making a few small wording changes. To help ensure content validity, I need a few subject matter experts to help in the next step, which is why I am reaching out to you again. I have attached a word document that includes the definitions for the 10 global leadership competencies in the framework and the questions intended to measure each competency. Would you take one more critical look at this document for me? Do you agree that the questions could measure that competency? Do you have any wording suggestions for any of the questions I might want to consider?

There is one catch... can you do this before the end of this week? I have cognitive interviews scheduled beginning Monday, so I need to incorporate any feedback I receive from this step prior to those interviews. If you will not have time, I completely understand and can reach out to others. I apologize that I need such a quick turn around, but trying to stay on track to graduate next Spring! Thanks in advance for your consideration.

Sincerely,

Sabrena 


\section{APPENDIX H}

Document sent to Experts for Final Consultation 


\section{O'Keefe Global Leadership Competencies Assessment Question Bank}

\section{Scale: Strongly Disagree, Disagree, Slightly Disagree, Slightly Agree, Agree,}

Strongly Agree

1. Responding to Change. Because of environmental needs, innovation, or matters out of one's own control, change can be sudden, frequent, and stressful. Leaders must be flexible and positive but must also be able to adapt quickly so that processes and procedures have a quick and smooth transition and are able to move forward and adjust to a new way of being.

a. I adjust my behavior when something occurs that is out of my control.

b. I implement a new plan when a change occurs.

c. I can be flexible when a change occurs.

2. Self-Development. Whether it is learning new technology or how to become a better public speaker, leaders are always learning.

a. I am committed to life-long learning.

b. I enjoy learning about new things.

c. I look for new opportunities to improve myself.

d. I expose myself to new experiences.

3. Responding to Ambiguity. Because leaders cannot truly control all circumstances and will never know all the answers, they must be able to respond to uncertainty and the unknown.

a. I react comfortably to ambiguity.

b. I remain open to new information in uncertain situations.

c. I respond calmly in ambiguous situations.

4. Others' Perspectives. Leaders do not have all the answers. Thus, it is essential that they are able to truly consider other opinions, experiences, and outlooks to help them develop better solutions and approaches when dealing with leadership situations.

a. I consider others' perspectives when making decisions.

b. I show interest in others' perspectives.

c. I am willing to adapt my perspective to incorporate others' perspectives when necessary.

5. Diversity. Leadership is inherently an interpersonal process, and in many situations, leaders will find themselves in the position of working with individuals who have different backgrounds, beliefs, and/or experiences than they do.

a. I am curious about different cultures.

b. I appreciate the differences of other cultures.

c. I enjoy exploring cultures other than my own.

d. I interact with individuals who have different backgrounds, beliefs, and/or experiences than I do. 
6. Productive Relationships. Leadership requires that a leader has meaningful connections with others; simply interacting with people does not constitute a relationship.

a. I am willing to take time to develop productive relationships.

b. I am building a network of people that can support and guide me.

c. I maintain productive relationships.

7. Empathy. Not only can demonstrating empathy with others build relationships and a sense of trust, it can also help a leader understand another point of view or other set of circumstances to effectively inform the leader's decisions and actions.

a. I emotionally connect with people who have experiences different from my own.

b. I put myself in others' situations.

c. I am empathetic toward others.

d. I consider other people's feelings when making decisions.

8. Self-Understanding. Self-awareness is vital to effective leadership. When a leader understands his or her feelings, beliefs, actions, skills, and personality, he or she can emphasize strengths and mitigate weaknesses in his or her leadership style and lead with more authenticity and in a more productive manner.

a. I am aware of my emotions during interactions with other people.

b. I understand my personal values and beliefs.

c. I understand my strengths and weaknesses.

9. Positive Attitude. Life is full of unexpected challenges, changes, and actions by others that can be discouraging or defeating. Attitude plays an important role in how one deals with these circumstances. A leader with a positive attitude can foster a sense of optimism, hope, inspiration, and enthusiasm even if the circumstances are bad.

a. I demonstrate a can-do attitude.

b. I maintain a positive outlook.

c. I remain positive in challenging situations.

d. I role model a positive attitude for others in difficult situations.

10. Resiliency. From the perspective of the leader, leadership does not always yield positive results; leaders face challenges and adversity that they cannot overcome, as well as major setbacks and disappointments. But it is the leader who can learn from the experience and rise again to the next challenge who stands out.

a. I quickly bounce back from failures.

b. I recover from setbacks.

c. I am resilient when things don't go the way I hoped. 


\section{APPENDIX I}

Qualtrics Form used for Cognitive Interviews 


\section{1st version OKeefe Global Leadership Assessment}

\section{Start of Block: Default Question Block}

Q1 FIU ADULT ONLINE CONSENT TO PARTICIPATE IN A RESEARCH

STUDY The Development of a Self-Assessment for Global Leadership

Competencies: A Validity Study

PURPOSE OF THE STUDY You are being asked to be in a research study. The purpose of this study is to develop a self-assessment instrument with acceptable reliability and validity inferences to measure global leadership competencies.

NUMBER OF STUDY PARTICIPANTS If you decide to be in this study, you will be one of at least 400 people in this research study.

DURATION OF THE STUDY Your participation will require approximately 20 minutes.

PROCEDURES If you agree to be in the study, we will ask you to complete the online assessment.

RISKS AND/OR DISCOMFORTS There are no anticipated risks associated with your participation in this study.

BENEFITS A potential benefit is at the end of the assessment there will be information about programs/activities that could be sought out if participants desire to increase any of the global leadership competencies measured.

ALTERNATIVES There are no known alternatives available to you other than not taking part in this study. However, any significant new findings developed during the course of the research which may relate to your willingness to continue participation will be provided to you.

CONFIDENTIALITY The records of this study will be kept private and will be protected to the fullest extent provided by law. In any sort of report we might publish, we will not include any information that will make it possible to identify a subject. Research records will be stored securely and only the researcher team will have access to the records. However, your records may be reviewed for audit purposes by authorized University or other agents who will be bound by the same provisions of confidentiality.

RIGHT TO DECLINE OR WITHDRAW Your participation in this study is voluntary. You are free to participate in the study or withdraw your consent at any time during the study. Your withdrawal or lack of participation will not affect any benefits to which you are otherwise entitled. The investigator reserves the right to remove you without your consent at such time that they feel it is in the best interest.

RESEARCHER CONTACT INFORMATION If you have any questions about the purpose, procedures, or any other issues relating to this research study you may contact Sabrena O'Keefe at 3000 NE 151st Street, WUC 353, North Miami, FL 33181,(305) 906-0789, saokeefe@fiu.edu.

IRB CONTACT INFORMATION If you would like to talk with someone about your rights of being a subject in this research study or about ethical issues with this research study, you may contact the FIU Office of Research Integrity by phone at 305-348-2494 or by email at ori@ fiu.edu.

PARTICIPANT AGREEMENT I have read the information in this consent form and agree to participate in this study. I have had a chance to ask any questions I 
have about this study, and they have been answered for me. By clicking on the "consent to participate" button below I am providing my informed consent.

Consent to Participate (1)

Page

Break

Q3 Current Class Standing

$\nabla$ First-Year (1) ... N/A (6)

Q5 Are you an international student?

No (1)

Yes from: (2)

Q7 Institution

If none, enter N/A

Q9 Major/Program of Study

If none, enter $N / A$

Q11 Are you Spanish, Hispanic, or Latino?

Yes (1)

None of these (2)

Q13 Choose one or more races that you consider yourself to be:

White (1)

Black or African American (2)

American Indian or Alaska Native (3)

Asian (4)

Native Hawaiian or Pacific Islander (5)

Other (6)

Q15 What is your gender?

Male (1) 
Female (2)

Other (3)

Q17 What is your age?

Under 18 (1)

18-24 (2)

25-34 (3)

$35+(4)$

Page

Break

Q20 I adjust my behavior when something occurs that is out of my control.

Strongly Disagree (1)

Disagree (2)

Slightly Disagree (3)

Slightly Agree (4)

Agree (5)

Strongly Agree (6)

Q12 I am committed to life-long learning.

Strongly Disagree (1)

Disagree (2)

Slightly Disagree (3)

Slightly Agree (4)

Agree (5)

Strongly Agree (6)

Q13 I react comfortably to uncertainty.

Strongly Disagree (1)

Disagree (2) 
Slightly Disagree (3)

Slightly Agree (4)

Agree (5)

Strongly Agree (6)

Q14 I consider others' perspectives when making decisions.

Strongly Disagree (1)

Disagree (2)

Slightly Disagree (3)

Slightly Agree (4)

Agree (5)

Strongly Agree (6)

Q15 I am curious about different cultures.

\section{Strongly Disagree (1)}

Disagree (2)

Slightly Disagree (3)

Slightly Agree (4)

Agree (5)

Strongly Agree (6)

Q16 I am willing to take time to develop productive relationships.

Strongly Disagree (1)

Disagree (2)

Slightly Disagree (3)

Slightly Agree (4)

Agree (5)

Strongly Agree (6) 
Q17 I emotionally connect with people who have experiences different from my own.

\section{Strongly Disagree (1)}

Disagree (2)

Slightly Disagree (3)

Slightly Agree (4)

Agree (5)

Strongly Agree (6)

Q18 I am aware of my emotions during interactions with other people.

Strongly Disagree (1)

Disagree (2)

Slightly Disagree (3)

Slightly Agree (4)

Agree (5)

Strongly Agree (6)

Q19 I demonstrate a can-do attitude.

Strongly Disagree (1)

Disagree (2)

Slightly Disagree (3)

Slightly Agree (4)

Agree (5)

Strongly Agree (6)

Q20 I quickly bounce back from failures.

Strongly Disagree (1)

Disagree (2)

Slightly Disagree (3) 
Slightly Agree (4)

Agree (5)

Strongly Agree (6)

Q21 I implement a new plan when a change occurs.

Strongly Disagree (1)

Disagree (2)

Slightly Disagree (3)

Slightly Agree (4)

Agree (5)

Strongly Agree (6)

Q22 I enjoy learning about new things.

Strongly Disagree (1)

Disagree (2)

Slightly Disagree (3)

Slightly Agree (4)

Agree (5)

Strongly Agree (6)

Q23 I remain open to new information in uncertain situations.

Strongly Disagree (1)

Disagree (2)

Slightly Disagree (3)

Slightly Agree (4)

Agree (5)

Strongly Agree (6)

Q24 I show interest in others' perspectives. 
Strongly Disagree (1)

Disagree (2)

Slightly Disagree (3)

Slightly Agree (4)

Agree (5)

Strongly Agree (6)

Q25 I appreciate the differences of other cultures.

Strongly Disagree (1)

Disagree (2)

Slightly Disagree (3)

Slightly Agree (4)

Agree (5)

Strongly Agree (6)

Q26 I am building a network of people that can support and guide me.

Strongly Disagree (1)

Disagree (2)

Slightly Disagree (3)

Slightly Agree (4)

Agree (5)

Strongly Agree (6)

Q27 I put myself in others' situations.

Strongly Disagree (1)

Disagree (2)

Slightly Disagree (3)

Slightly Agree (4) 
Agree (5)

Strongly Agree (6)

Q28 I understand my personal values and beliefs.

Strongly Disagree (1)

Disagree (2)

Slightly Disagree (3)

Slightly Agree (4)

Agree (5)

Strongly Agree (6)

Q29 I maintain a positive outlook.

Strongly Disagree (1)

Disagree (2)

Slightly Disagree (3)

Slightly Agree (4)

Agree (5)

Strongly Agree (6)

Q30 I recover from setbacks.

Strongly Disagree (1)

Disagree (2)

Slightly Disagree (3)

Slightly Agree (4)

Agree (5)

Strongly Agree (6)

Q31 I can be flexible when a change occurs.

Strongly Disagree (1) 
Disagree (2)

Slightly Disagree (3)

Slightly Agree (4)

Agree (5)

Strongly Agree (6)

Q32 I look for new opportunities to improve myself.

Strongly Disagree (1)

Disagree (2)

Slightly Disagree (3)

Slightly Agree (4)

Agree (5)

Strongly Agree (6)

Q33 I respond calmly in ambiguous situations.

Strongly Disagree (1)

Disagree (2)

Slightly Disagree (3)

Slightly Agree (4)

Agree (5)

Strongly Agree (6)

Q34 I am willing to adapt my perspective to incorporate others' perspectives when necessary.

Strongly Disagree (1)

Disagree (2)

Slightly Disagree (3)

Slightly Agree (4)

Agree (5) 
Strongly Agree (6)

Q35 I enjoy exploring cultures other than my own.

Strongly Disagree (1)

Disagree (2)

Slightly Disagree (3)

Slightly Agree (4)

Agree (5)

Strongly Agree (6)

Q36 I maintain productive relationships.

Strongly Disagree (1)

Disagree (2)

Slightly Disagree (3)

Slightly Agree (4)

Agree (5)

Strongly Agree (6)

Q37 I am empathetic toward others.

Strongly Disagree (1)

Disagree (2)

Slightly Disagree (3)

Slightly Agree (4)

Agree (5)

Strongly Agree (6)

Q38 I understand my strengths and weaknesses.

Strongly Disagree (1)

Disagree (2) 
Slightly Disagree (3)

Slightly Agree (4)

Agree (5)

Strongly Agree (6)

Q39 I remain positive in challenging situations.

Strongly Disagree (1)

Disagree (2)

Slightly Disagree (3)

Slightly Agree (4)

Agree (5)

Strongly Agree (6)

Q40 I am able to rise again when things don't go the way I hoped.

Strongly Disagree (1)

Disagree (2)

Slightly Disagree (3)

Slightly Agree (4)

Agree (5)

Strongly Agree (6)

Q41 I expose myself to new experiences.

Strongly Disagree (1)

Disagree (2)

Slightly Disagree (3)

Slightly Agree (4)

Agree (5)

Strongly Agree (6) 
Q42 I interact with individuals who have different backgrounds, beliefs, and/or experiences than I do.

Strongly Disagree (1)

Disagree (2)

Slightly Disagree (3)

Slightly Agree (4)

Agree (5)

Strongly Agree (6)

Q43 I consider other people's feelings when making decisions.

Strongly Disagree (1)

Disagree (2)

Slightly Disagree (3)

Slightly Agree (4)

Agree (5)

Strongly Agree (6)

Q44 I role model a positive attitude for others in difficult situations.

Strongly Disagree (1)

Disagree (2)

Slightly Disagree (3)

Slightly Agree (4)

Agree (5)

Strongly Agree (6)

Page

Break

Q46

Your Overall Global Leadership Score is \$e\{ round(\$\{gr://SC_8FYj6VjKv3QoVZb/WeightedMean\}, 2 ) \} (out of 6). 


\section{Here is how you scored on each of the individual Global Leadership Competencies (out of 6):}

\section{Diversity - \$e\{round( \$\{gr://SC_b3ijfkypCF8kgzr/WeightedMean\}, 2 ) \}}

Leadership is inherently an interpersonal process, and, in many situations, leaders will find themselves working with individuals who have different backgrounds, beliefs, and/or experiences.

Empathy - \$e $\{$ round(\$gr://SC_9z5MEVVviFVmRJr/WeightedMean $\}, 2)\}$ Demonstrating empathy toward others builds relationships and a sense of trust. It can also help a leader understand other points of view or sets of circumstances, effectively informing the leader's decisions and actions.

Others' Perspectives - $\$ \mathrm{e}\{\operatorname{round}(\$\{$ gr://SC_86Y18WytAiZlxYx/WeightedMean $\}$, 2 ) \}

Leaders do not have all of the answers. It is essential that they are able to truly consider other opinions, experiences, and outlooks to help them develop better solutions and approaches when dealing with leadership situations.

Positive Attitude - $\$ \mathrm{e}\{\operatorname{round}(\$\{$ gr://SC_0OFmE488rRt4aaN/WeightedMean $\}, 2$ ) $\}$ Life is full of unexpected challenges, changes, and actions by others that can be discouraging or defeating. Attitude plays an important role in how one deals with these circumstances. A leader with a positive attitude can foster a sense of optimism, hope, inspiration, and enthusiasm even if the circumstances are bad.

\section{Productive Relationships - $\$ \mathrm{e}\{$}

round (\$ gr://SC_bsCtRLffQDzaNSZ/WeightedMean \}, 2 ) \}

Leadership requires that a leader has meaningful connections with others; simply interacting with people does not constitute a relationship.

Resiliency - \$e $\{\operatorname{round}(\$\{$ gr://SC_3yk2UcMoe8EVVPv/WeightedMean $\}, 2)\}$ Leadership does not always yield positive results; leaders face major setbacks, disappointments, and challenges and adversity that they cannot overcome. Leaders who can learn from their experiences and rise to the next challenge stand out.

\section{Responding to Ambiguity - \$e \{}

round $(\$\{$ gr://SC_aayItH8JSZlnwtn/WeightedMean $\}, 2$ ) $\}$

Leaders must be able to respond to uncertainty and the unknown because they cannot truly control all circumstances and will never know all of the answers.

\section{Responding to Change - $\$ \mathrm{e}\{$}

round( $\$\{$ gr://SC_87kTP91QTpOadc9/WeightedMean $\}, 2$ ) $\}$

Change can be sudden, frequent, and stressful. Change may come about in response to environmental needs, innovation, or matters out of one's own control. Leaders must be flexible and positive but must also be able to adapt quickly so that processes 
and procedures transition quickly and smoothly. Leaders must be able to move forward and adjust to a new way of being.

Self-Development - \$e $\{$ round(\$gr://SC_6RqTubjNgggzShT/WeightedMean $\}, 2)\}$ Whether it is learning new technology or how to become a better public speaker, leaders are always learning.

\section{Self-Understanding - $\$ \mathrm{e}\left\{\operatorname{round}\left(\$\left\{\mathrm{gr}: / / \mathrm{SC} \_b N r M l p d h 81 \mathrm{JvGjr} /\right.\right.\right.$ WeightedMean $\left.\}, 2\right)$ \} \\ Self-awareness is vital to effective leadership. When a leader understands his or her feelings, beliefs, actions, skills, and personality, he or she can emphasize strengths and mitigate weaknesses in his or her leadership style and lead with more authenticity and in a more productive manner.}

\section{Note: Please print this page for your records. Your results are NOT saved for you to be able to review later.}

The definitions of the competencies above are from:

Seemiller, C. (2013). The student leadership competencies guidebook: Designing intentional leadership learning and development. Hoboken, NJ: John Wiley \& Sons.

Page

Break

Q46 O'Keefe Global Leadership Assessment Competency Development Examples

Here are just a few examples of resources that can help you continue to develop each of the different competencies that serve as the base for successful global leaders. It is meant to serve as a starting point for you to understand the types of activities that will increase each of the competencies for you. I encourage you to also look at co-curricular opportunities on your campus that will also increase these different areas.

Diversity - Leadership is inherently an interpersonal process, and, in many situations, leaders will find themselves working with individuals who have different backgrounds, beliefs, and/or experiences.

- Diversity Central: http://www.diversitycentral.com/

- Appreciating Diversity: http://www.collegesuccess 1.com/diversitym.htm

- TED Talks: https://www.ted.com/search?q=diversity

Empathy - Demonstrating empathy toward others builds relationships and a sense of trust. It can also help a leader understand other points of view or sets of circumstances, effectively informing the leader's decisions and actions.

- Empathy at Work: https://www.mindtools.com/pages/article/EmpathyatWork.htm

- How Empathic Are You: http://sfhelp.org/relate/empathy.htm

- Empathy Building Exercise: http://www.thoughtsfromatherapist.com/2011/06/08/empathy-building- 
exercise-\%E2\%80\%93-learning-to-be-empathetic-\%E2\%80\%93-increasingemotional-understanding/

Others' Perspectives - Leaders do not have all of the answers. It is essential that they are able to truly consider other opinions, experiences, and outlooks to help them develop better solutions and approaches when dealing with leadership situations.

- Dangers of a Single Story: http://www.npr.org/2013/09/20/186303292/what-arethe-dangers-of-a-single-story

- Perspective Taking: https://dash.harvard.edu/bitstream/handle/1/4556387/Gehlbach\%202009 $\%$ 20Social\%20Perspective\%20Taking.pdf?sequence $=1$

- Appreciative Inquiry: https://appreciativeinquiry.champlain.edu/

Q47 Positive Attitude. Life is full of unexpected challenges, changes, and actions by others that can be discouraging or defeating. Attitude plays an important role in how one deals with these circumstances. A leader with a positive attitude can foster a sense of optimism, hope, inspiration, and enthusiasm even if the circumstances are bad.

- Positive Thinking: https://www.mindtools.com/pages/article/newTCS_06.htm

- How to Create a Positive Attitude: https://www.inc.com/geoffrey-james/how-tocreate-a-positive-attitude.html

- 7 Practical Tips to Achieve a Positive

Mindset: https://www.success.com/article/7-practical-tips-to-achieve-a-positivemindset

Productive Relationships. Leadership requires that a leader has meaningful connections with others; simply interacting with people does not constitute a relationship.

- Building Great Work Relationships: https://www.mindtools.com/pages/article/good-relationships.htm

- How to build your network: https://hbr.org/2005/12/how-to-build-your-network

- How to Network Across Cultures: https://hbr.org/2012/01/how-to-networkacross-cultures

Resiliency. Leadership does not always yield positive results; leaders face major setbacks, disappointments, and challenges and adversity that they cannot overcome. Leaders who can learn from their experiences and rise to the next challenge stand out.

- Developing Resilience: https://www.mindtools.com/pages/article/resilience.htm

- Getting to grips with resilience: https://careerscaseload.com/getting-gripsresilience-part-2/

- The Road to Resilience: http://www.apa.org/helpcenter/road-resilience.aspx

Responding to Ambiguity. Leaders must be able to respond to uncertainty and the unknown because they cannot truly control all circumstances and will never know all of the answers.

- Managing in a VUCA

World: https://www.mindtools.com/pages/article/managing-vuca-world.htm 
- Dealing with Ambiguity: https://beyondphilosophy.com/dealing-with-ambiguitythe-new-business-imperative/

- Tolerating Ambiguity: https://www.youtube.com/watch?v=RZ0tS2vBEIA Q48 Responding to Change - Change can be sudden, frequent, and stressful. Change may come about in response to environmental needs, innovation, or matters out of one's own control. Leaders must be flexible and positive but must also be able to adapt quickly so that processes and procedures transition quickly and smoothly. Leaders must be able to move forward and adjust to a new way of being.

- Change Management: https://www.mindtools.com/pages/article/newPPM_87.htm

- Facilitating Change: https://www.lynda.com/Business-Skillstutorials/Facilitating-change/122471/139738-4.html

- 5 Tips to Help You Respond Effectively to Change: https://www.psychologytoday.com/blog/in-flux/201107/5-tips-help-yourespond-effectively-change

Self-Development. Whether it is learning new technology or how to become a better public speaker, leaders are always learning.

- Mindtools: https://www.mindtools.com/

- TED Talks: https://www.ted.com/topics

- Knowledge Lover: https://knowledgelover.com/learn-new-things-everyday/

Self-Understanding. Self-awareness is vital to effective leadership. When a leader understands his or her feelings, beliefs, actions, skills, and personality, he or she can emphasize strengths and mitigate weaknesses in his or her leadership style and lead with more authenticity and in a more productive manner.

- Developing Self Awareness: https://www.mindtools.com/pages/article/developing-self-awareness.htm

- Becoming aware of your own worldview: http://www.cultureadvantage.com/awarenesspage $2 . h$ tml

- Understand your own cultural awareness: http://www.culturosity.com/articles/whatisculturalawareness.htm

For a copy of this you can save and/or print click here. End of Block: Default Question Block 


\section{APPENDIX J}

\section{Cognitive Interview Process Guide}




\section{Cognitive Interview Process \\ Opening:}

1. Introduce yourself, thank the interviewee for coming, and show him/her where to sit.

2. Establish rapport with the interviewee to ease anxiety that s/he may have about participating in the cognitive interview.

3. Remind the interviewee about the purpose of the project and tell him/her you are interested in hearing what $\mathrm{s} / \mathrm{he}$ has to say about the materials.

4. "I will be tape recording the interview. Do I have your permission to record the interview?"

5. Answer any questions.

6. "Please remember that there are no wrong answers. I do not have a personal connection to any of the materials and you will not hurt my feelings. Feel free to say anything you're thinking."

\section{Warm-Up Introduction}

"Thinking aloud may be new and unfamiliar to you, but please know there are no wrong answers. I am only interested in knowing what is going through your mind. Before we begin the actual session, I'd like to ask you a 'warm-up' question to introduce you to the think aloud process.

'Try to visualize the place where you live, and think about how many windows there are in that place. As you count the windows, tell me what you are seeing and thinking about.' (Willis, 1994)"

\section{Interview}

"Please take this assessment, but use the think aloud process we just practiced as you do it."

\section{Possible Probes}

-Can you repeat the question you just read in your own words?

-What does ------- word mean to you?

-Explain why you gave yourself that score.

-How did you arrive at that answer?

-I noticed you were hesitating. Tell me what you were thinking.

-Tell me more about that.

\section{Closing}

"Thank you for taking time to answer these questions and for your participation in this project. Please feel free to share any other comments that you haven't shared to this point." 


\section{What to look for during this process:}

- Did the student understand the statement the way that the committee wants them to?

- Were there any words that seemed difficult to understand?

- Was there a word that was interpreted differently than intended?

- Did they hesitate while trying to recall / answer the question... why?

- Did they respond almost too fast, not giving the question actual consideration?

- Do they have suggestions on how to improve the wording of any questions?

- Were there questions that seemed the same in different sections (aka for different competencies)?

- Did they notice questions being the same in the same section and if so, did they mention preferring one over the other? 


\section{APPENDIX K}

\section{Emails to Recruit Pilot Participants}




\section{Email 1:}

The Development of a Self-Assessment for Global Leadership Competencies: A Validity Study

You are invited to participate in this research study aimed at developing a selfassessment instrument to measure global leadership competencies. The acceleration of globalization has created a need for an additional skill set not covered in the traditional leadership theories being call global leadership. The Student Leadership Competencies (www.studentleadershipcompetencies.com) is a body of research being used by universities across the nation to help connect out-of-classroom activities to learning outcomes that resonate across all academic disciplines. This assessment aims to combine those two concepts so that students have the opportunity to develop global leadership competencies on their own if their institution is unable to provide specific programming.

Students, student affairs practitioners, and employers will all potentially benefit from the development of this instrument. Students will benefit from the selfreflection of utilizing the instrument. Student affairs practitioners will benefit by having a tool to help students develop global leadership competencies. Finally, employers will benefit because more students will graduate with global leadership competency skills.

The data collected from this online assessment will be used to help establish reliability and validity inferences for the instrument. There are no risks beyond that of an individual's daily routine. A potential benefit is at the end of the assessment there is information about activities that you can use to begin increasing any of the global leadership competencies measured. You will also earn 5 bonus points added to the top of your grade (ie. if you have a 91... you will have a 96) but this must be completed by NOON on Tuesday, Nov. 7, $\underline{2017}$.

The assessment should not take more than 15 minutes. I thank you in advance for your willingness to participate in our research study.

Sincerely, go.fiu.edu/GLAssessment

Sabrena O'Keefe

\section{Email 2:}

In order to get the extra credit for taking the global leadership assessment just sent to you, you must screenshot or print \& scan the results page and email it to me by Tues., Nov. 7 at noon. The assessment does not collect identifying information.

Sincerely,

Sabrena 


\section{APPENDIX L}

Qualtrics form used during Final Phase of Data Collection 


\section{3rd version OKeefe Global Leadership Assessment}

Start of Block: Default Question Block

\section{Q49 The Development of a Self-Assessment for Global Leadership}

\section{Competencies: A Validity Study}

You are invited to participate in this research study aimed at developing a selfassessment instrument to measure global leadership competencies. The acceleration of globalization has created a need for an additional skill set not covered in the traditional leadership theories being call global leadership. The Student Leadership Competencies (www.studentleadershipcompetencies.com) is a body of research being used by universities across the nation to help connect out-of-classroom activities to learning outcomes that resonate across all academic disciplines. This assessment aims to combine those two concepts so that students have the opportunity to develop global leadership competencies on their own if their institution is unable to provide specific programming.

Students, student affairs practitioners, and employers will all potentially benefit from the development of this instrument. Students will benefit from the self-reflection of utilizing the instrument. Student affairs practitioners will benefit by having a tool to help students develop global leadership competencies. Finally, employers will benefit because more students will graduate with global leadership competency skills. The data collected from this online assessment will be used to help establish reliability and validity inferences for the instrument. There are no risks beyond that of an individual's daily routine. A potential benefit is at the end of the assessment there is examples of activities that you could seek out if you desire to increase any of the global leadership competencies measured after you receive your results. The assessment should not take more than 20 minutes. I thank you in advance for your willingness to participate in our research study and encourage you to send this information to anyone you think might be interested in also participating.

Q1 FIU ADULT ONLINE CONSENT TO PARTICIPATE IN A RESEARCH STUDY The Development of a Self-Assessment for Global Leadership Competencies: A Validity Study

PURPOSE OF THE STUDY You are being asked to be in a research study. The purpose of this study is to develop a self-assessment instrument with acceptable reliability and validity inferences to measure global leadership competencies. NUMBER OF STUDY PARTICIPANTS If you decide to be in this study, you will be one of at least 400 people in this research study.

DURATION OF THE STUDY Your participation will require approximately 20 minutes.

PROCEDURES If you agree to be in the study, we will ask you to complete the online assessment.

RISKS AND/OR DISCOMFORTS There are no anticipated risks associated with your participation in this study.

BENEFITS A potential benefit is at the end of the assessment there will be information about programs/activities that could be sought out if participants desire to increase any of the global leadership competencies measured. 
ALTERNATIVES There are no known alternatives available to you other than not taking part in this study. However, any significant new findings developed during the course of the research which may relate to your willingness to continue participation will be provided to you.

CONFIDENTIALITY The records of this study will be kept private and will be protected to the fullest extent provided by law. In any sort of report we might publish, we will not include any information that will make it possible to identify a subject. Research records will be stored securely and only the researcher team will have access to the records. However, your records may be reviewed for audit purposes by authorized University or other agents who will be bound by the same provisions of confidentiality.

RIGHT TO DECLINE OR WITHDRAW Your participation in this study is voluntary. You are free to participate in the study or withdraw your consent at any time during the study. Your withdrawal or lack of participation will not affect any benefits to which you are otherwise entitled. The investigator reserves the right to remove you without your consent at such time that they feel it is in the best interest. RESEARCHER CONTACT INFORMATION If you have any questions about the purpose, procedures, or any other issues relating to this research study you may contact Sabrena O'Keefe at 3000 NE 151st Street, WUC 353, North Miami, FL 33181, (305) 906-0789, saokeefe@ fiu.edu.

IRB CONTACT INFORMATION If you would like to talk with someone about your rights of being a subject in this research study or about ethical issues with this research study, you may contact the FIU Office of Research Integrity by phone at 305-348-2494 or by email at ori@fiu.edu.

PARTICIPANT AGREEMENT I have read the information in this consent form and agree to participate in this study. I have had a chance to ask any questions I have about this study, and they have been answered for me. By clicking on the "consent to participate" button below I am providing my informed consent.

Consent to Participate (1)

Page

Break

Q3 Current Class Standing

$\nabla$ First-Year (1) ... N/A (6)

Q5 Are you an international student?

No (1)

Yes from: (2)

Q7 Institution

If none, enter N/A 
Q9 Major/Program of Study

If none, enter N/A

Q11 Are you Spanish, Hispanic, or Latino?

Yes (1)

None of these (2)

Q13 Choose one or more races that you consider yourself to be:

White (1)

Black or African American (2)

American Indian or Alaska Native (3)

Asian (4)

Native Hawaiian or Pacific Islander (5)

Other (6)

Q15 What is your gender?

Male (1)

Female (2)

Other (3)

Q17 What is your age?

Under 18 (1)

18-24 (2)

25-34 (3)

$35+(4)$

Page

Break

Q20 I adjust my behavior when something occurs that is out of my control.

Strongly Disagree (1)

Disagree (2) 
Slightly Disagree (3)

Slightly Agree (4)

Agree (5)

Strongly Agree (6)

Q12 I am committed to life-long learning.

Strongly Disagree (1)

Disagree (2)

Slightly Disagree (3)

Slightly Agree (4)

Agree (5)

Strongly Agree (6)

Q13 I react comfortably to uncertainty.

Strongly Disagree (1)

Disagree (2)

Slightly Disagree (3)

Slightly Agree (4)

Agree (5)

Strongly Agree (6)

Q14 I consider others' perspectives when making decisions.

Strongly Disagree (1)

Disagree (2)

Slightly Disagree (3)

Slightly Agree (4)

Agree (5)

Strongly Agree (6) 
Q15 I am curious about different cultures.

Strongly Disagree (1)

Disagree (2)

Slightly Disagree (3)

Slightly Agree (4)

Agree (5)

Strongly Agree (6)

Q16 I am willing to take time to develop productive relationships.

Strongly Disagree (1)

Disagree (2)

Slightly Disagree (3)

Slightly Agree (4)

Agree (5)

Strongly Agree (6)

Q17 I emotionally connect with people who have experiences different from my own.

Strongly Disagree (1)

Disagree (2)

Slightly Disagree (3)

Slightly Agree (4)

Agree (5)

Strongly Agree (6)

Q18 I am aware of my emotions during interactions with other people.

Strongly Disagree (1)

Disagree (2)

Slightly Disagree (3) 
Slightly Agree (4)

Agree (5)

Strongly Agree (6)

Q19 I demonstrate a can-do attitude.

Strongly Disagree (1)

Disagree (2)

Slightly Disagree (3)

Slightly Agree (4)

Agree (5)

Strongly Agree (6)

Q20 I quickly bounce back from failures.

Strongly Disagree (1)

Disagree (2)

Slightly Disagree (3)

Slightly Agree (4)

Agree (5)

Strongly Agree (6)

Q21 I implement a new plan when a change occurs.

Strongly Disagree (1)

Disagree (2)

Slightly Disagree (3)

Slightly Agree (4)

Agree (5)

Strongly Agree (6)

Q23 I remain open to new information in uncertain situations. 
Strongly Disagree (1)

Disagree (2)

Slightly Disagree (3)

Slightly Agree (4)

Agree (5)

Strongly Agree (6)

Q24 I show interest in others' perspectives.

Strongly Disagree (1)

Disagree (2)

Slightly Disagree (3)

Slightly Agree (4)

Agree (5)

Strongly Agree (6)

Q25 I appreciate the differences of other cultures.

Strongly Disagree (1)

Disagree (2)

Slightly Disagree (3)

Slightly Agree (4)

Agree (5)

Strongly Agree (6)

Q26 I am building a network of people that can support and guide me.

Strongly Disagree (1)

Disagree (2)

Slightly Disagree (3)

Slightly Agree (4) 
Agree (5)

Strongly Agree (6)

Q27 I put myself in others' situations.

Strongly Disagree (1)

Disagree (2)

Slightly Disagree (3)

Slightly Agree (4)

Agree (5)

Strongly Agree (6)

Q28 I understand my personal values and beliefs.

Strongly Disagree (1)

Disagree (2)

Slightly Disagree (3)

Slightly Agree (4)

Agree (5)

Strongly Agree (6)

Q30 I recover from setbacks.

Strongly Disagree (1)

Disagree (2)

Slightly Disagree (3)

Slightly Agree (4)

Agree (5)

Strongly Agree (6)

Q31 I can be flexible when a change occurs.

Strongly Disagree (1) 
Disagree (2)

Slightly Disagree (3)

Slightly Agree (4)

Agree (5)

Strongly Agree (6)

Q32 I look for new opportunities to improve myself.

Strongly Disagree (1)

Disagree (2)

Slightly Disagree (3)

Slightly Agree (4)

Agree (5)

Strongly Agree (6)

Q33 I respond calmly in ambiguous situations.

Strongly Disagree (1)

Disagree (2)

Slightly Disagree (3)

Slightly Agree (4)

Agree (5)

Strongly Agree (6)

Q34 I am willing to adapt my perspective to incorporate others' perspectives when necessary.

Strongly Disagree (1)

Disagree (2)

Slightly Disagree (3)

Slightly Agree (4)

Agree (5) 
Strongly Agree (6)

Q35 I enjoy exploring cultures other than my own.

Strongly Disagree (1)

Disagree (2)

Slightly Disagree (3)

Slightly Agree (4)

Agree (5)

Strongly Agree (6)

Q36 I maintain productive relationships.

Strongly Disagree (1)

Disagree (2)

Slightly Disagree (3)

Slightly Agree (4)

Agree (5)

Strongly Agree (6)

Q37 I am empathetic toward others.

Strongly Disagree (1)

Disagree (2)

Slightly Disagree (3)

Slightly Agree (4)

Agree (5)

Strongly Agree (6)

Q38 I understand my strengths and weaknesses.

Strongly Disagree (1)

Disagree (2) 
Slightly Disagree (3)

Slightly Agree (4)

Agree (5)

Strongly Agree (6)

Q39 I remain positive in challenging situations.

Strongly Disagree (1)

Disagree (2)

Slightly Disagree (3)

Slightly Agree (4)

Agree (5)

Strongly Agree (6)

Q40 I am able to rise again when things don't go the way I hoped.

Strongly Disagree (1)

Disagree (2)

Slightly Disagree (3)

Slightly Agree (4)

Agree (5)

Strongly Agree (6)

Q41 I expose myself to new experiences.

Strongly Disagree (1)

Disagree (2)

Slightly Disagree (3)

Slightly Agree (4)

Agree (5)

Strongly Agree (6) 
Q44 I role model a positive attitude for others in difficult situations.

Strongly Disagree (1)

Disagree (2)

Slightly Disagree (3)

Slightly Agree (4)

Agree (5)

Strongly Agree (6)

Page

Break

Q46

Your Overall Global Leadership Score is \$e\{

round(\$gr://SC_8FYj6VjKv3QoVZb/WeightedMean\}, 2 ) \} (out of 6).

\section{Here is how you scored on each of the individual Global Leadership Competencies (out of 6):}

Diversity - \$e\{round( \$\{gr://SC_b3ijfkypCF8kgzr/WeightedMean\}, 2 ) \}

Leadership is inherently an interpersonal process, and, in many situations, leaders will find themselves working with individuals who have different backgrounds, beliefs, and/or experiences.

Empathy - \$e\{round(\$gr://SC_9z5MEVVviFVmRJr/WeightedMean $\}, 2$ ) \} Demonstrating empathy toward others builds relationships and a sense of trust. It can also help a leader understand other points of view or sets of circumstances, effectively informing the leader's decisions and actions.

Others' Perspectives - $\$ \mathrm{e}\{\operatorname{round}(\$\{$ gr://SC_86Y18WytAiZlxYx/WeightedMean $\}$, 2) \}

Leaders do not have all of the answers. It is essential that they are able to truly consider other opinions, experiences, and outlooks to help them develop better solutions and approaches when dealing with leadership situations.

Positive Attitude - \$e $\{$ round(\$gr://SC_0OFmE488rRt4aaN/WeightedMean\}, 2 ) \} Life is full of unexpected challenges, changes, and actions by others that can be discouraging or defeating. Attitude plays an important role in how one deals with these circumstances. A leader with a positive attitude can foster a sense of optimism, hope, inspiration, and enthusiasm even if the circumstances are bad. 
Productive Relationships - \$e \{

round( $\$\{$ gr://SC_bsCtRLffQDzaNSZ/WeightedMean $\}, 2$ ) $\}$

Leadership requires that a leader has meaningful connections with others; simply interacting with people does not constitute a relationship.

Resiliency - \$e $\{\operatorname{round}(\$\{$ gr://SC_3yk2UcMoe8EVVPv/WeightedMean $\}, 2)\}$ Leadership does not always yield positive results; leaders face major setbacks, disappointments, and challenges and adversity that they cannot overcome. Leaders who can learn from their experiences and rise to the next challenge stand out.

\section{Responding to Ambiguity - $\$ \mathrm{e}\{$}

round( $\$\{$ gr://SC_aayItH8JSZlnwtn/WeightedMean $\}, 2$ ) $\}$

Leaders must be able to respond to uncertainty and the unknown because they cannot truly control all circumstances and will never know all of the answers.

\section{Responding to Change - $\$ \mathrm{e}\{$} round $(\$\{$ gr://SC_87kTP91QTpOadc9/WeightedMean $\}, 2$ ) $\}$

Change can be sudden, frequent, and stressful. Change may come about in response to environmental needs, innovation, or matters out of one's own control. Leaders must be flexible and positive but must also be able to adapt quickly so that processes and procedures transition quickly and smoothly. Leaders must be able to move forward and adjust to a new way of being.

Self-Development - \$e $\{\operatorname{round}(\$\{$ gr://SC_6RqTubjNgggzShT/WeightedMean $\}, 2$ ) $\}$ Whether it is learning new technology or how to become a better public speaker, leaders are always learning.

Self-Understanding-\$e $\{\operatorname{round}(\$\{$ gr://SC_bNrMlpdh81JvGjr/WeightedMean $\}, 2$ ) $\}$ Self-awareness is vital to effective leadership. When a leader understands his or her feelings, beliefs, actions, skills, and personality, he or she can emphasize strengths and mitigate weaknesses in his or her leadership style and lead with more authenticity and in a more productive manner.

Note: Please print this page for your records. Your results are NOT saved for you to be able to review later.

The definitions of the competencies above are from:

Seemiller, C. (2013). The student leadership competencies guidebook: Designing intentional leadership learning and development. Hoboken, NJ: John Wiley \& Sons. Page

Break

Q46 O’Keefe Global Leadership Assessment

Competency Development Examples

Here are just a few examples of resources that can help you continue to develop each of the different competencies that serve as the base for successful global leaders. It is 
meant to serve as a starting point for you to understand the types of activities that will increase each of the competencies for you. I encourage you to also look at co-

curricular opportunities on your campus that will also increase these different areas.

Diversity - Leadership is inherently an interpersonal process, and, in many situations, leaders will find themselves working with individuals who have different backgrounds, beliefs, and/or experiences.

- Diversity Central: http://www.diversitycentral.com/

- Appreciating Diversity: http://www.collegesuccess 1.com/diversitym.htm

- TED Talks: https://www.ted.com/search?q=diversity

Empathy - Demonstrating empathy toward others builds relationships and a sense of trust. It can also help a leader understand other points of view or sets of circumstances, effectively informing the leader's decisions and actions.

- Empathy at Work: https://www.mindtools.com/pages/article/EmpathyatWork.htm

- How Empathic Are You: http://sfhelp.org/relate/empathy.htm

- Empathy Building Exercise: http://www.thoughtsfromatherapist.com/2011/06/08/empathy-buildingexercise-\%E2\%80\%93-learning-to-be-empathetic-\%E2\%80\%93-increasingemotional-understanding/

Others' Perspectives - Leaders do not have all of the answers. It is essential that they are able to truly consider other opinions, experiences, and outlooks to help them develop better solutions and approaches when dealing with leadership situations.

- Dangers of a Single Story: http://www.npr.org/2013/09/20/186303292/whatare-the-dangers-of-a-single-story

- Perspective Taking: https://dash.harvard.edu/bitstream/handle/1/4556387/Gehlbach\%202009\%20S ocial\%20Perspective\%20Taking.pdf? sequence $=1$

- Appreciative Inquiry: https://appreciativeinquiry.champlain.edu/

Q47 Positive Attitude. Life is full of unexpected challenges, changes, and actions by others that can be discouraging or defeating. Attitude plays an important role in how one deals with these circumstances. A leader with a positive attitude can foster a sense of optimism, hope, inspiration, and enthusiasm even if the circumstances are bad.

- Positive Thinking: https://www.mindtools.com/pages/article/newTCS_06.htm

- How to Create a Positive Attitude: https://www.inc.com/geoffrey-james/howto-create-a-positive-attitude.html

- 7 Practical Tips to Achieve a Positive Mindset: https://www.success.com/article/7-practical-tips-to-achieve-apositive-mindset 
Productive Relationships. Leadership requires that a leader has meaningful connections with others; simply interacting with people does not constitute a relationship.

- Building Great Work Relationships:

https://www.mindtools.com/pages/article/good-relationships.htm

- How to build your network: https://hbr.org/2005/12/how-to-build-yournetwork

- How to Network Across Cultures: https://hbr.org/2012/01/how-to-networkacross-cultures

Resiliency. Leadership does not always yield positive results; leaders face major setbacks, disappointments, and challenges and adversity that they cannot overcome. Leaders who can learn from their experiences and rise to the next challenge stand out.

- Developing Resilience: https://www.mindtools.com/pages/article/resilience.htm

- Getting to grips with resilience: https://careerscaseload.com/getting-gripsresilience-part-2/

- The Road to Resilience: http://www.apa.org/helpcenter/road-resilience.aspx

Responding to Ambiguity. Leaders must be able to respond to uncertainty and the unknown because they cannot truly control all circumstances and will never know all of the answers.

- Managing in a VUCA World: https://www.mindtools.com/pages/article/managing-vuca-world.htm

- Dealing with Ambiguity: https://beyondphilosophy.com/dealing-withambiguity-the-new-business-imperative/

- Tolerating Ambiguity: https://www.youtube.com/watch?v=RZ0tS2vBEIA

Q48 Responding to Change - Change can be sudden, frequent, and stressful. Change may come about in response to environmental needs, innovation, or matters out of one's own control. Leaders must be flexible and positive but must also be able to adapt quickly so that processes and procedures transition quickly and smoothly. Leaders must be able to move forward and adjust to a new way of being.

- Change Management: https://www.mindtools.com/pages/article/newPPM_87.htm

- Facilitating Change: https://www.lynda.com/Business-Skillstutorials/Facilitating-change/122471/139738-4.html

- 5 Tips to Help You Respond Effectively to Change: https://www.psychologytoday.com/blog/in-flux/201107/5-tips-helpyou-respond-effectively-change 
Self-Development. Whether it is learning new technology or how to become a better public speaker, leaders are always learning.

- Mindtools: https://www.mindtools.com/

- TED Talks: https://www.ted.com/topics

- Knowledge Lover: https://knowledgelover.com/learn-new-things-everyday/

Self-Understanding. Self-awareness is vital to effective leadership. When a leader understands his or her feelings, beliefs, actions, skills, and personality, he or she can emphasize strengths and mitigate weaknesses in his or her leadership style and lead with more authenticity and in a more productive manner.

- Developing Self Awareness:

https://www.mindtools.com/pages/article/developing-self-awareness.htm

- Becoming aware of your own worldview: http://www.cultureadvantage.com/awarenesspage $2 . \mathrm{html}$

- Understand your own cultural awareness:

http://www.culturosity.com/articles/whatisculturalawareness.htm

For a copy of this you can save and/or print click here. End of Block: Default Question Block 


\section{APPENDIX M}

First Email to Professional Contacts 


\title{
Greetings,
}

I hope you had a wonderful Thanksgiving break! As you may recall, I am working on my dissertation "The Development of a Self-Assessment for Global Leadership Competencies: A Validity Study." I am now in my final phase of collecting data, which means I need your assistance. I am looking to obtain a diverse sample from across the nation. Could you forward the link below to your students? Include it as extra credit? Build it in as an addition to one of your programs? Send it over any listservs you can? Maybe just take it yourself? Any of those would be helpful! Here is a little more information about it:

- The survey is designed to measure 10 different competencies that have been identified as the basis of global leadership success

- Survey needs completed by end of Fall 2017 (I know it's quick... but hopefully I'll have a final product for you to use after the Spring semester!)

- Can be completed in approximately 20 minutes or less

- Results are given automatically at the end (note: this does not save for them to return to, so students need to record their results)

- Examples of activities to engage in that help increase the competencies is provided at the end as well

Again, any way you are able to distribute and/or encourage students to take this assessment would be appreciated. I also welcome feedback about the instrument if you have any!

Here is the link, which has both a brief explanation of the purpose of instrument and a consent to participate at the beginning to make sharing it easier.

\section{go.fiu.edu/OKeefeGLA}

Thanks in advance!

Sincerely,

Sabrena

\author{
Sabrena O'Keefe \\ Associate Director \\ Center for Leadership \& Service \\ Florida International University - BBC \\ 3000 N.E. 151 st St \\ WUC 353 \\ North Miami, FL 33181 \\ 305.919.5360 \\ leadserve.fiu.edu
}




\section{APPENDIX N}

First Facebook Recruitment Post 
You know that dissertation I'm working on? Well I'm into my final phase of data collection and YOU can help! The link below will take you to a brief description of the study, a consent to participate, and the Global Leadership Assessment. While I'm targeting a diverse national pool of undergraduate college students, anybody can check it out and take it!

go.fiu.edu/OKeefeGLA 
APPENDIX O

Graphic for Recruitment of Participants 


\section{DISCOVER YOUR CLOBAL \\ LEADERSHIP \\ COMPETENCIES SCORE!}

\section{GO.FIU.EDU/OKEEFEGLA}

There are 10 leadership competencies that serve as the base for any successful global leader... find out how you rank and receive

examples of activities to help increase them. 


\section{APPENDIX P}

Second Recruitment Email to Professional Contacts 


\section{Greetings!}

I just wanted to send a friendly reminder that I am in need of some assistance with data collection for my dissertation. If each of you could take the instrument yourself and get at least 1 (someone suggested I say 3, but I will seriously take whatever I can get!) student, I will have the numbers I need! Please note that I am currently collecting data from undergraduate students, graduate students, already graduated, never went to college... literally everyone! If you are a decisive person, it will only take 5 mins and if you like to think a little longer on questions it has not taken more than 15 mins to take.... Plus you get a result instantly! http://go.fiu.edu/okeefegla

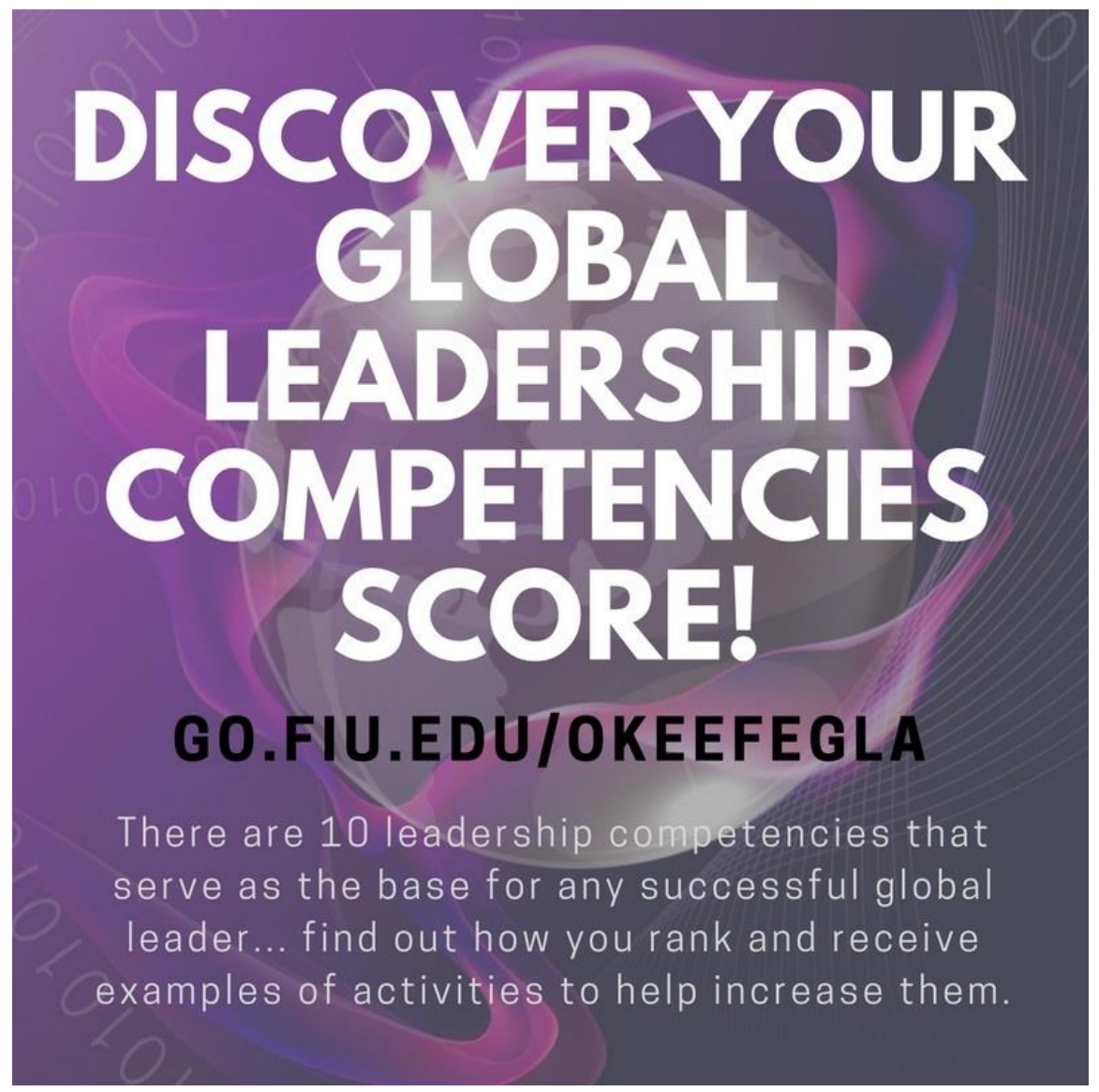

I know it is finals and the holidays, which is not a very convenient time, but I need this data by the end of 2017 in order to be able to graduate in the Spring. Thank you in advance for however you are able to help!

Sincerely, Sabrena 


\section{APPENDIX Q}

Post in Student Affairs Professional Facebook Group 
Greetings! I'm in need of some assistance with data collection for my dissertation. I am currently collecting data from undergraduate students, graduate students, already graduated, never went to college... literally everyone! If you are a decisive person, it will only take 5 mins and if you like to think a little longer on questions it has not taken more than 15 mins to take.... Plus you get a result instantly! I know it is finals and the holidays, which is not a very convenient time, but I need this data by the end of 2017 in order to be able to graduate in the Spring. Thank you in advance for however you are able to help! http://go.fiu.edu/okeefegla

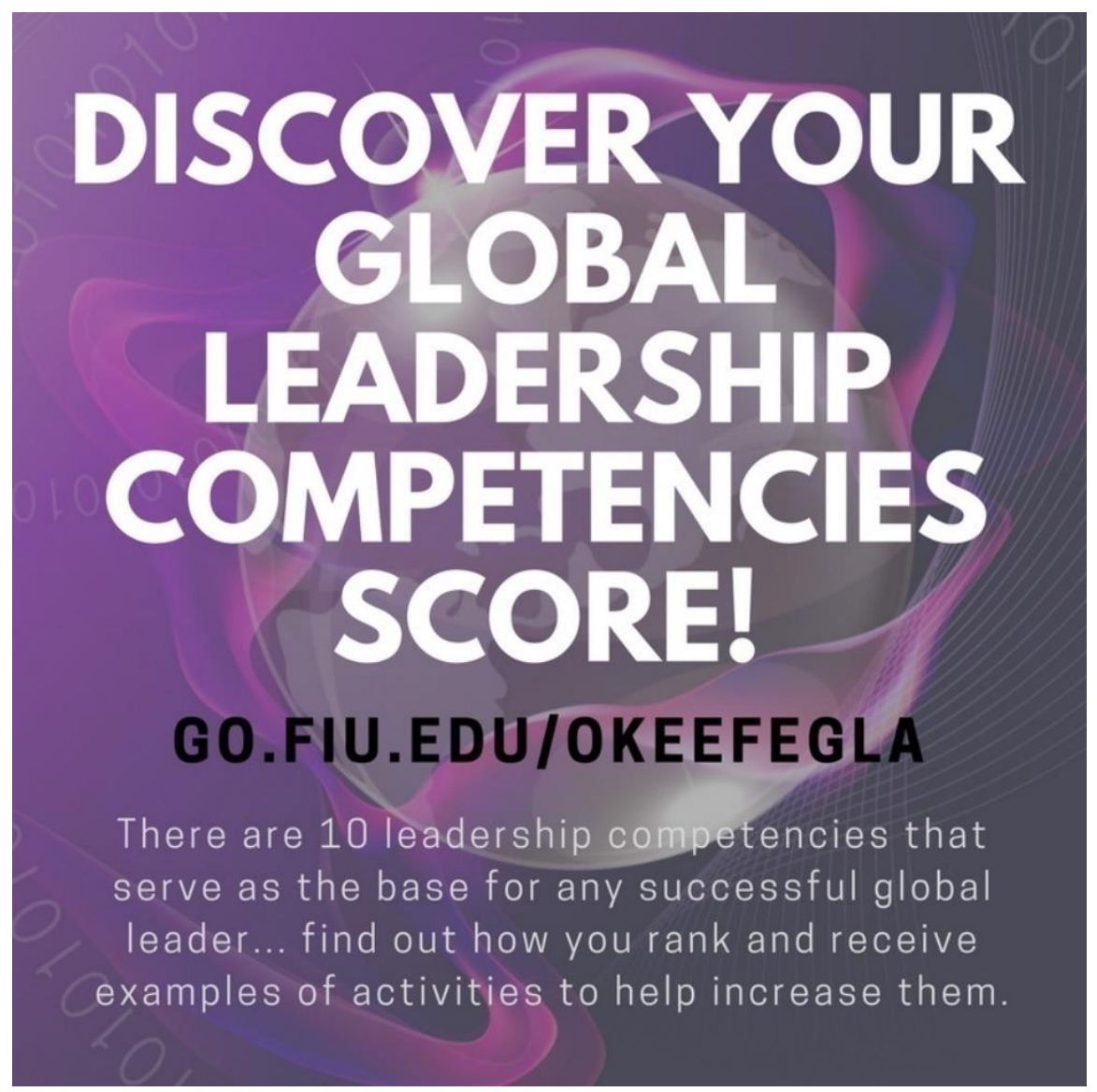




\section{APPENDIX R}

Institutions of Higher Education Represented in Phase 4 
Institutions of Higher Education

1. Abraham Lincoln University

2. California State University- Dominguez Hills

3. Colorado State University

4. Florida International University (157)

5. Indiana University of Pennsylvania

6. Kingsborough Community College (9)

7. Lycoming College (2)

8. Miami Dade College

9. Minnesota State University - Mankato, N/A (22)

10. Norfolk State University

11. North Carolina Central University

12. Northern Kentucky University (24)

13. Penn Foster College

14. Pennsylvania State University

15. San Jose State

16. Stockton University

17. Texas A\&M University - San Antonio (2)

18. Texas Christian University (25)

19. Texas State University (6)

20. University of Alabama (2)

21. University of California Irvine

22. University of Florida (15)

23. University of Iowa

24. University of Miami (3)

25. University of South Florida (6)

26. University of Texas at Austin (4)

27. University of Virginia

28. Washington State University

29. Wright State University 


\section{APPENDIX S}

Visual Overview of Subject Matter Experts Comment Color-coding on the Table of Specifications Tab 1 


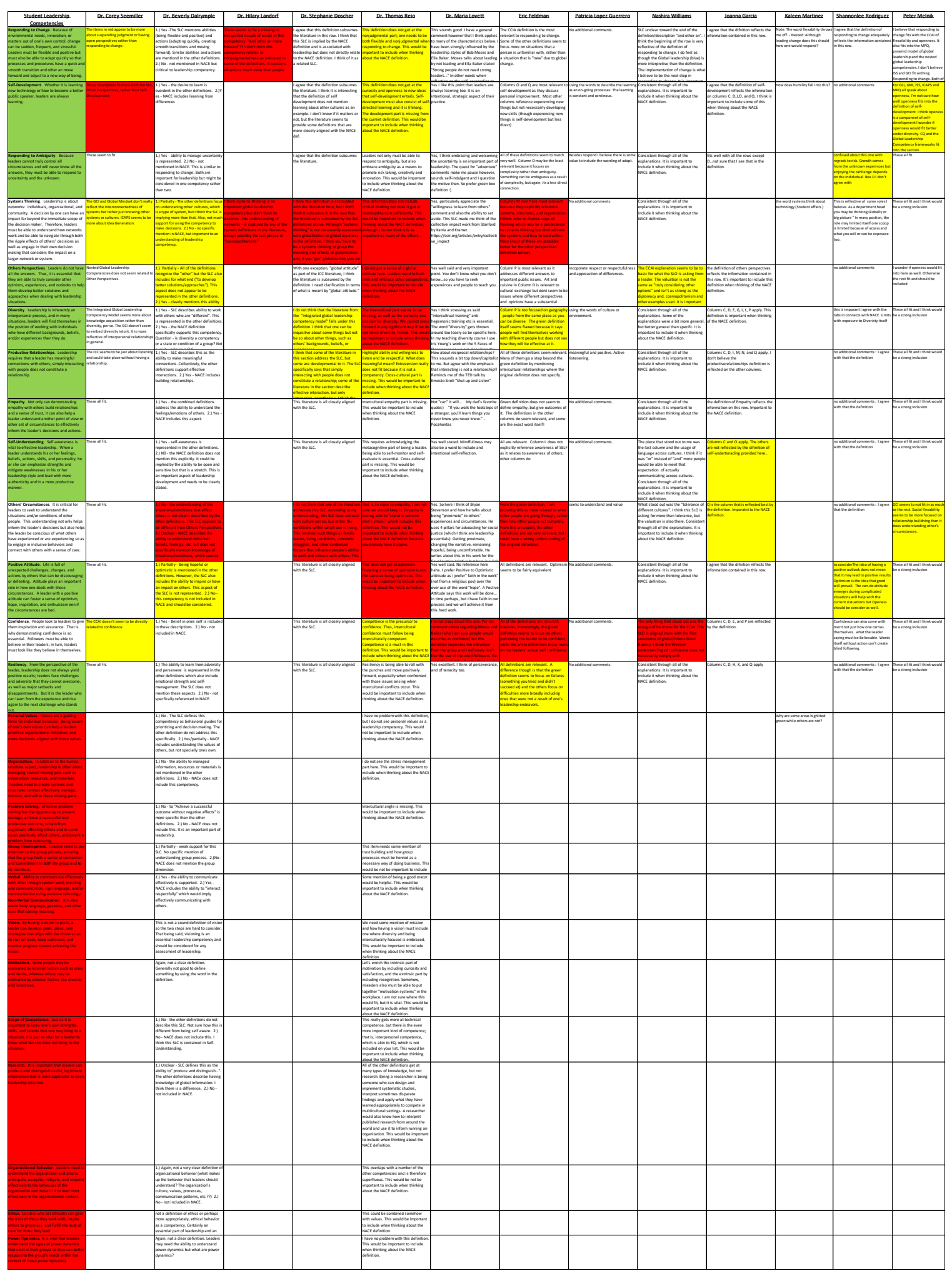




\section{APPENDIX T}

Visual Overview of Subject Matter Experts' Tallies Color-coding on the Table of Specifications Tab 2 


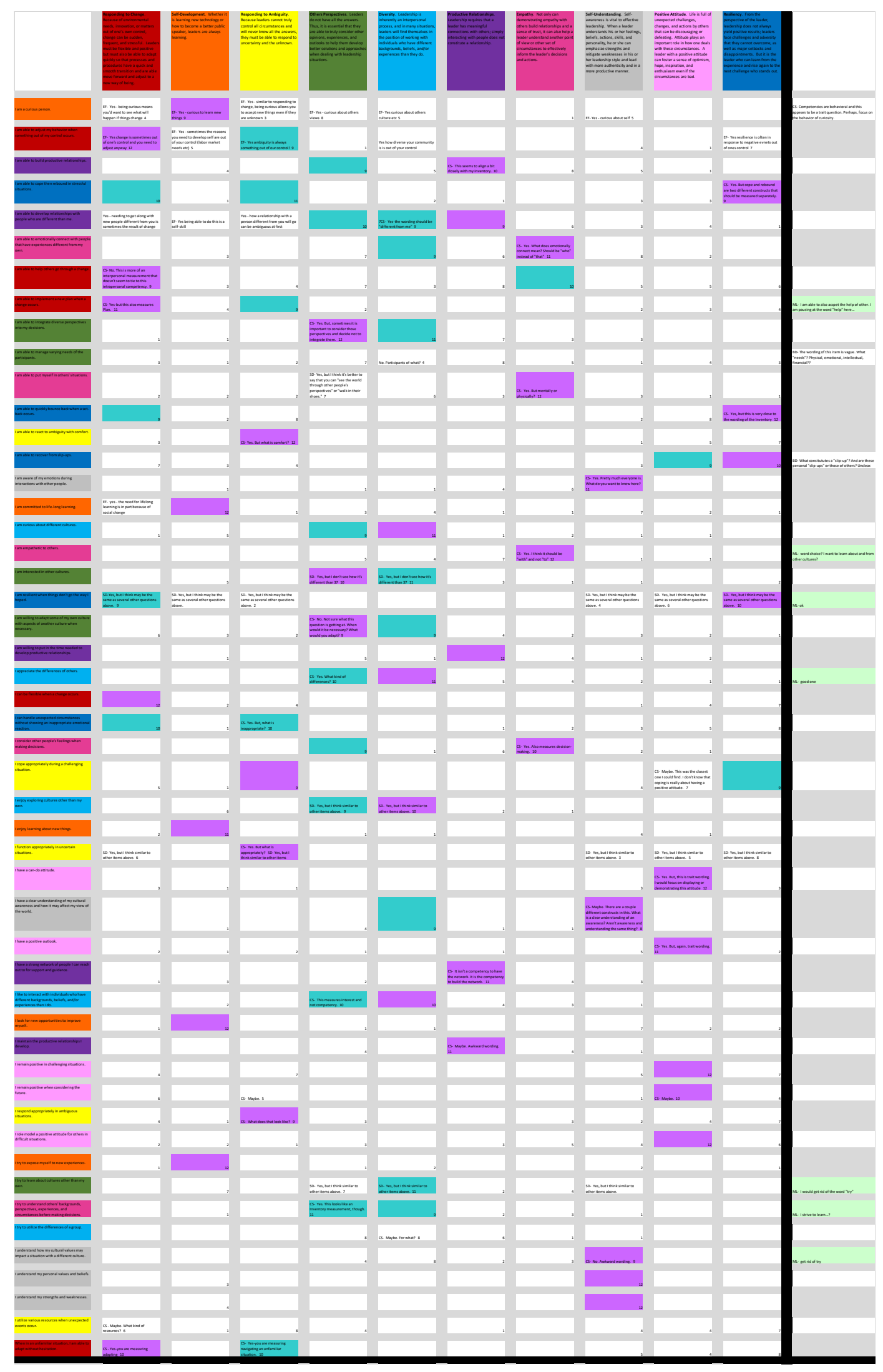


APPENDIX U

Visual Overview of Sorted Tab 2 


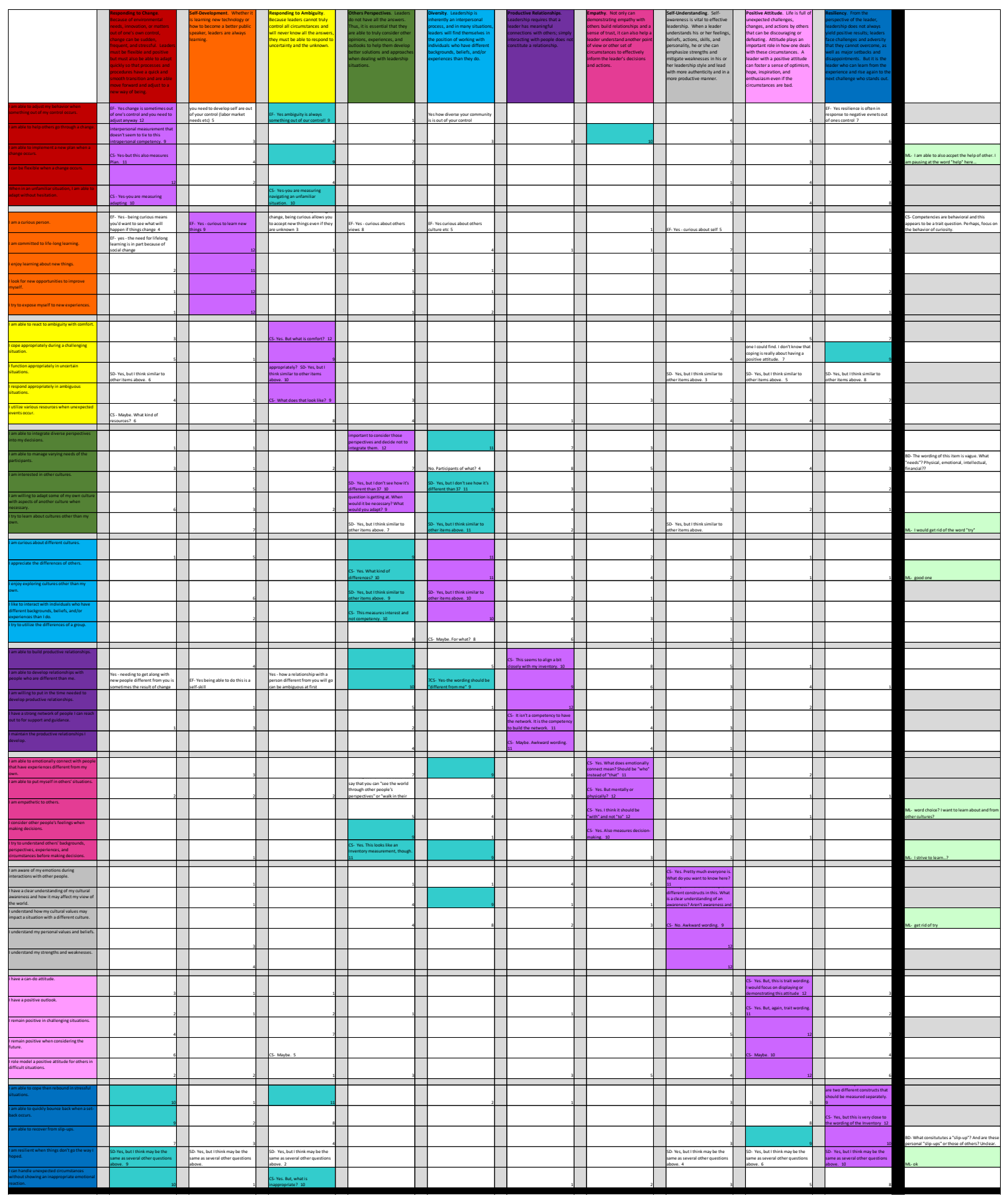




\section{APPENDIX V}

Revised Questions after Cognitive Interviews 


\section{O'Keefe Global Leadership Competencies Assessment Question Bank}

\section{Scale: Strongly Disagree, Disagree, Slightly Disagree, Slightly Agree, Agree, Strongly Agree}

1. Responding to Change. Because of environmental needs, innovation, or matters out of one's own control, change can be sudden, frequent, and stressful. Leaders must be flexible and positive but must also be able to adapt quickly so that processes and procedures have a quick and smooth transition and are able to move forward and adjust to a new way of being.

a. I adjust my behavior when something occurs that is out of my control.

b. I implement a new plan when a change occurs.

c. I can be flexible when a change occurs.

2. Self-Development. Whether it is learning new technology or how to become a better public speaker, leaders are always learning.

a. I am committed to life-long learning.

b. I enjoy learning about new things.

c. I look for new opportunities to improve myself.

d. I expose myself to new experiences.

3. Responding to Ambiguity. Because leaders cannot truly control all circumstances and will never know all the answers, they must be able to respond to uncertainty and the unknown.

a. I react comfortably to uncertainty.

b. I remain open to new information in uncertain situations.

c. I respond calmly in ambiguous situations.

4. Others' Perspectives. Leaders do not have all the answers. Thus, it is essential that they are able to truly consider other opinions, experiences, and outlooks to help them develop better solutions and approaches when dealing with leadership situations.

a. I consider others' perspectives when making decisions.

b. I show interest in others' perspectives.

c. I am willing to adapt my perspective to incorporate others' perspectives when necessary.

5. Diversity. Leadership is inherently an interpersonal process, and in many situations, leaders will find themselves in the position of working with individuals who have different backgrounds, beliefs, and/or experiences than they do.

a. I am curious about different cultures.

b. I appreciate the differences of other cultures.

c. I enjoy exploring cultures other than my own.

d. I interact with individuals who have different backgrounds, beliefs, and/or experiences than I do. 
6. Productive Relationships. Leadership requires that a leader has meaningful connections with others; simply interacting with people does not constitute a relationship.

a. I am willing to take time to develop productive relationships.

b. I am building a network of people that can support and guide me.

c. I maintain productive relationships.

7. Empathy. Not only can demonstrating empathy with others build relationships and a sense of trust, it can also help a leader understand another point of view or other set of circumstances to effectively inform the leader's decisions and actions.

a. I emotionally connect with people who have experiences different from my own.

b. I put myself in others' situations.

c. I am empathetic toward others.

d. I consider other people's feelings when making decisions.

8. Self-Understanding. Self-awareness is vital to effective leadership. When a leader understands his or her feelings, beliefs, actions, skills, and personality, he or she can emphasize strengths and mitigate weaknesses in his or her leadership style and lead with more authenticity and in a more productive manner.

a. I am aware of my emotions during interactions with other people.

b. I understand my personal values and beliefs.

c. I understand my strengths and weaknesses.

9. Positive Attitude. Life is full of unexpected challenges, changes, and actions by others that can be discouraging or defeating. Attitude plays an important role in how one deals with these circumstances. A leader with a positive attitude can foster a sense of optimism, hope, inspiration, and enthusiasm even if the circumstances are bad.

a. I demonstrate a can-do attitude.

b. I maintain a positive outlook.

c. I remain positive in challenging situations.

d. I role model a positive attitude for others in difficult situations.

10. Resiliency. From the perspective of the leader, leadership does not always yield positive results; leaders face challenges and adversity that they cannot overcome, as well as major setbacks and disappointments. But it is the leader who can learn from the experience and rise again to the next challenge who stands out.

a. I quickly bounce back from failures.

b. I recover from setbacks.

c. I am able to rise again when things don't go the way I hoped. 


\section{APPENDIX W}

Revised Questions after Pilot Study 


\section{O'Keefe Global Leadership Competencies Assessment Question Bank}

\section{Scale: Strongly Disagree, Disagree, Slightly Disagree, Slightly Agree, Agree,}

Strongly Agree

1. Responding to Change. Because of environmental needs, innovation, or matters out of one's own control, change can be sudden, frequent, and stressful. Leaders must be flexible and positive but must also be able to adapt quickly so that processes and procedures have a quick and smooth transition and are able to move forward and adjust to a new way of being.

a. I adjust my behavior when something occurs that is out of my control.

b. I implement a new plan when a change occurs.

c. I can be flexible when a change occurs.

2. Self-Development. Whether it is learning new technology or how to become a better public speaker, leaders are always learning.

a. I am committed to life-long learning.

b. I look for new opportunities to improve myself.

c. I expose myself to new experiences.

3. Responding to Ambiguity. Because leaders cannot truly control all circumstances and will never know all the answers, they must be able to respond to uncertainty and the unknown.

a. I react comfortably to uncertainty.

b. I remain open to new information in uncertain situations.

c. I respond calmly in ambiguous situations.

4. Others' Perspectives. Leaders do not have all the answers. Thus, it is essential that they are able to truly consider other opinions, experiences, and outlooks to help them develop better solutions and approaches when dealing with leadership situations.

a. I consider others' perspectives when making decisions.

b. I show interest in others' perspectives.

c. I am willing to adapt my perspective to incorporate others' perspectives when necessary.

5. Diversity. Leadership is inherently an interpersonal process, and in many situations, leaders will find themselves in the position of working with individuals who have different backgrounds, beliefs, and/or experiences than they do.

a. I am curious about different cultures.

b. I appreciate the differences of other cultures.

c. I enjoy exploring cultures other than my own. 
6. Productive Relationships. Leadership requires that a leader has meaningful connections with others; simply interacting with people does not constitute a relationship.

a. I am willing to take time to develop productive relationships.

b. I am building a network of people that can support and guide me.

c. I maintain productive relationships.

7. Empathy. Not only can demonstrating empathy with others build relationships and a sense of trust, it can also help a leader understand another point of view or other set of circumstances to effectively inform the leader's decisions and actions.

a. I emotionally connect with people who have experiences different from my own.

b. I put myself in others' situations.

c. I am empathetic toward others.

8. Self-Understanding. Self-awareness is vital to effective leadership. When a leader understands his or her feelings, beliefs, actions, skills, and personality, he or she can emphasize strengths and mitigate weaknesses in his or her leadership style and lead with more authenticity and in a more productive manner.

a. I am aware of my emotions during interactions with other people.

b. I understand my personal values and beliefs.

c. I understand my strengths and weaknesses.

9. Positive Attitude. Life is full of unexpected challenges, changes, and actions by others that can be discouraging or defeating. Attitude plays an important role in how one deals with these circumstances. A leader with a positive attitude can foster a sense of optimism, hope, inspiration, and enthusiasm even if the circumstances are bad.

a. I demonstrate a can-do attitude.

b. I remain positive in challenging situations.

c. I role model a positive attitude for others in difficult situations.

10. Resiliency. From the perspective of the leader, leadership does not always yield positive results; leaders face challenges and adversity that they cannot overcome, as well as major setbacks and disappointments. But it is the leader who can learn from the experience and rise again to the next challenge who stands out.

a. I quickly bounce back from failures.

b. I recover from setbacks.

c. I am able to rise again when things don't go the way I hoped. 
APPENDIX X

Final Instrument in Qualtrics 


\section{4th version OKeefe Global Leadership Assessment}

\section{Start of Block: Default Question Block}

Q49 The Development of a Self-Assessment for Global Leadership Competencies: A Validity Study

You are invited to participate in this research study aimed at developing a selfassessment instrument to measure global leadership competencies. The acceleration of globalization has created a need for an additional skill set not covered in the traditional leadership theories being call global leadership. The Student Leadership Competencies (www.studentleadershipcompetencies.com) is a body of research being used by universities across the nation to help connect out-of-classroom activities to learning outcomes that resonate across all academic disciplines. This assessment aims to combine those two concepts so that students have the opportunity to develop global leadership competencies on their own if their institution is unable to provide specific programming. Students, student affairs practitioners, and employers will all potentially benefit from the development of this instrument. Students will benefit from the self-reflection of utilizing the instrument. Student affairs practitioners will benefit by having a tool to help students develop global leadership competencies. Finally, employers will benefit because more students will graduate with global leadership competency skills.

The data collected from this online assessment will be used to help establish reliability and validity inferences for the instrument. There are no risks beyond that of an individual's daily routine. A potential benefit is at the end of the assessment there is examples of activities that you could seek out if you desire to increase any of the global leadership competencies measured after you receive your results.

The assessment should not take more than 20 minutes. I thank you in advance for your willingness to participate in our research study and encourage you to send this information to anyone you think might be interested in also participating.

Q1 FIU ADULT ONLINE CONSENT TO PARTICIPATE IN A RESEARCH STUDY The Development of a Self-Assessment for Global Leadership Competencies: A Validity Study

PURPOSE OF THE STUDY You are being asked to be in a research study. The purpose of this study is to develop a self-assessment instrument with acceptable reliability and validity inferences to measure global leadership competencies. NUMBER OF STUDY PARTICIPANTS If you decide to be in this study, you will be one of at least 400 people in this research study.

DURATION OF THE STUDY Your participation will require approximately 20 minutes.

PROCEDURES If you agree to be in the study, we will ask you to complete the online assessment.

RISKS AND/OR DISCOMFORTS There are no anticipated risks associated with your participation in this study. 
BENEFITS A potential benefit is at the end of the assessment there will be information about programs/activities that could be sought out if participants desire to increase any of the global leadership competencies measured.

ALTERNATIVES There are no known alternatives available to you other than not taking part in this study. However, any significant new findings developed during the course of the research which may relate to your willingness to continue participation will be provided to you.

CONFIDENTIALITY The records of this study will be kept private and will be protected to the fullest extent provided by law. In any sort of report we might publish, we will not include any information that will make it possible to identify a subject. Research records will be stored securely and only the researcher team will have access to the records. However, your records may be reviewed for audit purposes by authorized University or other agents who will be bound by the same provisions of confidentiality.

RIGHT TO DECLINE OR WITHDRAW Your participation in this study is voluntary. You are free to participate in the study or withdraw your consent at any time during the study. Your withdrawal or lack of participation will not affect any benefits to which you are otherwise entitled. The investigator reserves the right to remove you without your consent at such time that they feel it is in the best interest.

RESEARCHER CONTACT INFORMATION If you have any questions about the purpose, procedures, or any other issues relating to this research study you may contact Sabrena O'Keefe at 3000 NE 151st Street, WUC 353, North Miami, FL 33181, (305) 906-0789, saokeefe@fiu.edu.

IRB CONTACT INFORMATION If you would like to talk with someone about your rights of being a subject in this research study or about ethical issues with this research study, you may contact the FIU Office of Research Integrity by phone at 305-348-2494 or byemailatori@fiu.edu.

PARTICIPANT AGREEMENT I have read the information in this consent form and agree to participate in this study. I have had a chance to ask any questions I have about this study, and they have been answered for me. By clicking on the "consent to participate" button below I am providing my informed consent.

Consent to Participate (1)

Page Break

Q3 Current Class Standing

$\nabla$ First-Year (1) ... N/A (6)

Q5 Are you an international student?

No (1)

Yes from: (2)

Q7 Institution (ie. Florida International University)

If none, enter N/A 
Q9 Major/Program of Study (ie. Hospitality Management)

If none, enter N/A

Q11 Are you Spanish, Hispanic, or Latino?

Yes (1)

None of these (2)

Q13 Choose one or more races that you consider yourself to be:

White (1)

Black or African American (2)

American Indian or Alaska Native (3)

Asian (4)

Native Hawaiian or Pacific Islander (5)

Other (6)

Q15 What is your gender?

Male (1)

Female (2)

Trans (M-F) (4)

Trans (F-M) (5)

Other (3)

Choose not to disclose (6)

Q17 What is your age?

Under 18 (1)

18-24 (2)

25-34 (3)

$35+(4)$

Page Break 
Q20 I adjust my behavior when something occurs that is out of my control.

Strongly Disagree (1)

Disagree (2)

Slightly Disagree (3)

Slightly Agree (4)

Agree (5)

Strongly Agree (6)

Q12 I am committed to life-long learning.

Strongly Disagree (1)

Disagree (2)

Slightly Disagree (3)

Slightly Agree (4)

Agree (5)

Strongly Agree (6)

Q13 I react comfortably to uncertainty.

Strongly Disagree (1)

Disagree (2)

Slightly Disagree (3)

Slightly Agree (4)

Agree (5)

Strongly Agree (6)

Q14 I consider others' perspectives when making decisions.

Strongly Disagree (1)

Disagree (2)

Slightly Disagree (3)

Slightly Agree (4) 
Agree (5)

Strongly Agree (6)

Q15 I am curious about different cultures.

Strongly Disagree (1)

Disagree (2)

Slightly Disagree (3)

Slightly Agree (4)

Agree (5)

Strongly Agree (6)

Q16 I am willing to take time to develop productive relationships.

Strongly Disagree (1)

Disagree (2)

Slightly Disagree (3)

Slightly Agree (4)

Agree (5)

Strongly Agree (6)

Q17 I emotionally connect with people who have experiences different from my own.

Strongly Disagree (1)

Disagree (2)

Slightly Disagree (3)

Slightly Agree (4)

Agree (5)

Strongly Agree (6)

Q18 I am aware of my emotions during interactions with other people.

Strongly Disagree (1) 
Disagree (2)

Slightly Disagree (3)

Slightly Agree (4)

Agree (5)

Strongly Agree (6)

Q19 I demonstrate a can-do attitude.

Strongly Disagree (1)

Disagree (2)

Slightly Disagree (3)

Slightly Agree (4)

Agree (5)

Strongly Agree (6)

Q20 I quickly bounce back from failures.

Strongly Disagree (1)

Disagree (2)

Slightly Disagree (3)

Slightly Agree (4)

Agree (5)

Strongly Agree (6)

Q21 I implement a new plan when a change occurs.

Strongly Disagree (1)

Disagree (2)

Slightly Disagree (3)

Slightly Agree (4)

Agree (5) 
Strongly Agree (6)

Q23 I remain open to new information in uncertain situations.

Strongly Disagree (1)

Disagree (2)

Slightly Disagree (3)

Slightly Agree (4)

Agree (5)

Strongly Agree (6)

Q24 I show interest in others' perspectives.

Strongly Disagree (1)

Disagree (2)

Slightly Disagree (3)

Slightly Agree (4)

Agree (5)

Strongly Agree (6)

Q25 I appreciate the differences of other cultures.

Strongly Disagree (1)

Disagree (2)

Slightly Disagree (3)

Slightly Agree (4)

Agree (5)

Strongly Agree (6)

Q26 I am building a network of people that can support and guide me.

Strongly Disagree (1)

Disagree (2) 
Slightly Disagree (3)

Slightly Agree (4)

Agree (5)

Strongly Agree (6)

Q27 I put myself in others' situations.

Strongly Disagree (1)

Disagree (2)

Slightly Disagree (3)

Slightly Agree (4)

Agree (5)

Strongly Agree (6)

Q28 I understand my personal values and beliefs.

Strongly Disagree (1)

Disagree (2)

Slightly Disagree (3)

Slightly Agree (4)

Agree (5)

Strongly Agree (6)

Q30 I recover from setbacks.

Strongly Disagree (1)

Disagree (2)

Slightly Disagree (3)

Slightly Agree (4)

Agree (5)

Strongly Agree (6) 
Q31 I can be flexible when a change occurs.

Strongly Disagree (1)

Disagree (2)

Slightly Disagree (3)

Slightly Agree (4)

Agree (5)

Strongly Agree (6)

Q32 I look for new opportunities to improve myself.

Strongly Disagree (1)

Disagree (2)

Slightly Disagree (3)

Slightly Agree (4)

Agree (5)

Strongly Agree (6)

Q33 I respond calmly in ambiguous situations.

Strongly Disagree (1)

Disagree (2)

Slightly Disagree (3)

Slightly Agree (4)

Agree (5)

Strongly Agree (6)

Q34 I am willing to adapt my perspective to incorporate others' perspectives when necessary.

Strongly Disagree (1)

Disagree (2)

Slightly Disagree (3) 
Slightly Agree (4)

Agree (5)

Strongly Agree (6)

Q35 I enjoy exploring cultures other than my own.

Strongly Disagree (1)

Disagree (2)

Slightly Disagree (3)

Slightly Agree (4)

Agree (5)

Strongly Agree (6)

Q36 I maintain productive relationships.

Strongly Disagree (1)

Disagree (2)

Slightly Disagree (3)

Slightly Agree (4)

Agree (5)

Strongly Agree (6)

Q37 I am empathetic toward others.

Strongly Disagree (1)

Disagree (2)

Slightly Disagree (3)

Slightly Agree (4)

Agree (5)

Strongly Agree (6)

Q38 I understand my strengths and weaknesses. 
Strongly Disagree (1)

Disagree (2)

Slightly Disagree (3)

Slightly Agree (4)

Agree (5)

Strongly Agree (6)

Q39 I remain positive in challenging situations.

Strongly Disagree (1)

Disagree (2)

Slightly Disagree (3)

Slightly Agree (4)

Agree (5)

Strongly Agree (6)

Q40 I am able to rise again when things don't go the way I hoped.

Strongly Disagree (1)

Disagree (2)

Slightly Disagree (3)

Slightly Agree (4)

Agree (5)

Strongly Agree (6)

Q41 I expose myself to new experiences.

Strongly Disagree (1)

Disagree (2)

Slightly Disagree (3)

Slightly Agree (4) 
Agree (5)

Strongly Agree (6)

Q44 I role model a positive attitude for others in difficult situations.

Strongly Disagree (1)

Disagree (2)

Slightly Disagree (3)

Slightly Agree (4)

Agree (5)

Strongly Agree (6)

Page Break

Q46

Your Overall Global Leadership Score

is $\$ \mathrm{e}\{$ round(\$gr://SC_8FYj6VjKv3QoVZb/WeightedMean\}, 2 ) \} (out of 6).

Here is how you scored on each of the individual Global Leadership Competencies (out of 6):

Adapting - \$e\{round(\$gr://SC_87kTP91QTpOadc9/WeightedMean\}, 2 ) \} A leader must have the ability to be open to new information, though the type of information differed, and then adapt as necessary. Reference Responding to Change and Self-Development activities.

Diversity - \$e round(\$gr://SC_b3ijfkypCF8kgzr/WeightedMean\}, 2 ) \}

Leadership is inherently an interpersonal process, and, in many situations, leaders will find themselves working with individuals who have different backgrounds, beliefs, and/or experiences. Reference Diversity actvities.

\section{Interpersonal Impact - \$e\{round(\$\{gr://SC_bsCtRLffQDzaNSZ/WeightedMean\}, 2} ) $\}$

A leader's positive outlook and actions create the ability to develop strong productive relationships. Reference Productive Relationships and Positive Attitude activities.

Perspective-taking - \$e\{round(\$\{gr://SC_86Y18WytAiZlxYx/WeightedMean\}, 2 ) \} A leader must first be aware of where they stand on an issue, be conscious of how others may be feeling regarding that issue, then take the time to understand perspectives different from their own prior to deciding on the issue. Reference Self-Understanding, Empathy, and Others' Perspectives activities. 


\section{Resiliency - \$e\{round(\$gr://SC_3yk2UcMoe8EVVPv/WeightedMean\}, 2 ) \}}

Leadership does not always yield positive results; leaders face major setbacks, disappointments, and challenges and adversity that they cannot overcome. Leaders who can learn from their experiences and rise to the next challenge stand out. Reference Resiliency activities.

\section{Responding to Ambiguity - \$e\{ \\ round(\$\{gr://SC_aayItH8JSZInwtn/WeightedMean\}, 2 ) \}}

Leaders must be able to respond to uncertainty and the unknown because they cannot truly control all circumstances and will never know all of the answers. Reference Responding to Ambiguity activities.

Note: Please print this page for your records. Your results are NOT saved for you to be able to review later.

You can find out more about referenced Student Leadership Competencies (Seemiller, 2013) and activities at www.studentleadershipcompetencies.com. 
VITA

SABRENA O'KEEFE

2002-2004

2004-2007

2007-2009

2010-2011

2011-2016

2012-2015

2016-present

$2016-2018$
A.A.S., Computer Networking Pennsylvania College of Technology Williamsport, Pennsylvania

B.S., Human Resource Management Pennsylvania College of Technology Williamsport, Pennsylvania

M.Ed., Higher Education and Student Affairs University of South Carolina

Columbia, South Carolina

Assistant Director

Leadership and Commuter Student Services

Fordham University

Bronx, New York

Assistant Director

Center for Leadership and Service, BBC

Florida International University

North Miami, Florida

Graduate Certificate, Conflict Resolution and Consensus Building

Florida International University

Miami, Florida

Associate Director

Center for Leadership and Service, BBC

Florida International University

North Miami, Florida

Instructor

Florida International University

Miami, Florida

Doctoral Candidate

Florida International University

Miami, Florida 


\section{PUBLICATIONS AND PRESENTATIONS}

O'Keefe, S. (2009, Sept.). Opening the Door to Diversity. Campus Activities Programming, p. 46 - 49.

O'Keefe, S. (2009, Jan. 9). Creating a Tool to Help Your Advisees Become Engaged on Your Campus. The Mentor: An Academic Advising Journal, http://www.psu.edu/dus/mentor/.

O'Keefe, S. (2017, Jun). Development and Use of Pre/Post Assessments to Help Demonstrate Student Learning. Presentation at NASPA Assessment and Persistence Conference, Orlando, FL.

O'Keefe, S. \& Garcia, J. (2017, Mar.). "Welcome to the Big Leagues": Transforming Traditional Leadership Programming to Online. Presented at NASPA Annual Conference, San Antonio, TX.

O’Keefe, S. \& Jamanca, S. (2015, Oct.). Connecting Student Affairs to Academic Affairs through Student Leadership Competencies. Presented at the NASPA FL Drive-in Conference, Tampa, FL.

O’Keefe, S. \& Martinez, K. (2016, Oct.). Demonstrating Competence Through SelfReflection. Presented at NASPA FL Drive-in Conference, Miami, FL.

O’Keefe, S., Montgomery, K., \& Carral, C. (2017, Oct.). Implementing a Division-Wide Student Learning Outcomes Program to Enhance Career Readiness for Students. Presentation at the NASPA FL Drive-in Conference, Gainesville, FL.

O’Keefe, S. \& Ortiz, A. (2015, Dec.). Global Leadership Brigade: Utilizing international and domestic students to teach global leadership topics. Presented at the NASPA Multicultural Institute, Miami, FL.

O’Keefe, S. \& Seemiller, C. (2016, Mar). Bridging Student Affairs and Academic Affairs Using the Student Leadership Competencies. Presented at NASPA Annual Conference, Indianapolis, IN.

Seemiller, C. \& O’Keefe, S. (2016). Student leadership competencies program design handbook. Beavercreek, OH: LeadU, LLC.

Seemiller, C., O’Keefe, S., Ashby, K., Mintner, P., Lamp, D., Brown, J., Baumhardt, M., \& Bapasola, E. (2016, Mar.). Promising Partnerships: Implementing Student Leadership Competencies for Intentional Student Development. Presented at the NASPA Annual Conference Full Day Pre-Conference, Indianapolis, IN. 